\title{
Ricci curvature and convergence of Lipschitz functions
}

\author{
SHOUHEI HONDA
}

\begin{abstract}
We give the definition of a convergence of the differentials of Lipschitz functions with respect to the measured Gromov-Hausdorff topology and several properties of the convergence.
\end{abstract}

\section{Introduction}

Let $\left\{\left(M_{i}, m_{i}\right)\right\}_{i \in \mathbf{N}}$ be a sequence of pointed $n$-dimensional complete Riemannian manifolds $(n \geq 2)$ with $\operatorname{Ric}_{M_{i}} \geq-(n-1)$, and $(Y, y, v)$ a pointed proper metric space (i.e., every bounded subset of $Y$ is relatively compact) with a Radon measure $v$ on $Y$ satisfying that $\left(M_{i}, m_{i}, \underline{\text { vol }}\right)$ converges to $(Y, y, v)$ with respect to the measured Gromov-Hausdorff topology. Here vol is the renormalized Riemannian volume of $\left(M_{i}, m_{i}\right)$ : $\underline{\operatorname{vol}}=\mathrm{vol} / \operatorname{vol} B_{1}\left(m_{i}\right)$. Fix $R>0$, a sequence $\{f\}_{1 \leq i<\infty}$ of Lipschitz functions $f_{i}$ on $B_{R}\left(m_{i}\right)=\{w \in$ $\left.M_{i} ; \overline{w, m_{i}}<R\right\}$, and a Lipschitz function $f_{\infty}$ on $B_{R}(y)$ with $\sup _{i} \operatorname{Lip} f_{i}<\infty$. Here $\overline{w, m_{i}}$ is the distance between $w$ and $m_{i}, \operatorname{Lip} f_{i}$ is the Lipschitz constant of $f_{i}$. Then we say that $f_{i}$ converges to $f_{\infty}$ on $B_{R}(y)$ if $f_{i}\left(x_{i}\right) \rightarrow f_{\infty}\left(x_{\infty}\right)$ for every $x_{i} \in B_{R}\left(m_{i}\right)$ and every $x_{\infty} \in B_{R}(y)$ satisfying that $x_{i}$ converges to $x_{\infty}$. See Section 2 for these precise definitions. Assume that $f_{i}$ converges to $f_{\infty}$ on $B_{R}(y)$, below.

The purpose of this paper is to give a definition: the differentials $d f_{i}$ of $f_{i}$ converges to the differential $d f_{\infty}$ of $f_{\infty}$ in this setting. In order to give the definition below, we shall recall celebrated works on limit spaces of Riemannian manifolds by Cheeger-Colding. By [1] and [6], it is known that the cotangent bundle $T^{*} Y$ of $Y$ exists. We remark that each fiber $T_{w}^{*} Y$ is a finite-dimensional real vector space with canonical inner product $\langle\cdot, \cdot\rangle(w)$ for a.e. $w \in Y$, and that every Lipschitz function $g$ on $B_{R}(y)$ has the canonical differential section: $d g(w) \in T_{w}^{*} Y$ for a.e. $w \in B_{R}(y)$. See Section 4 in [1] and Section 6 in [6] for the details.

\footnotetext{
2000 Mathematics Subject Classification. Primary 53C20; Secondary 53C43.
} 
We shall give the definition of a convergence of the differentials of Lipschitz functions (see Definition 4.4):

Definition 1.1 Convergence of the differentials of Lipschitz functions. We say that $d f_{i}$ converges to $d f_{\infty}$ on $B_{R}(y)$ if for every $\epsilon>0$, every $x_{\infty} \in B_{R}(y)$, every $z_{\infty} \in Y$, every sequence $\left\{x_{i}\right\}_{1 \leq i<\infty}$ of points $x_{i} \in$ $B_{R}\left(m_{i}\right)$ satisfying that $x_{i}$ converges to $x_{\infty}$, and every sequence $\left\{z_{i}\right\}_{1 \leq i<\infty}$ of points $z_{i} \in M_{i}$ satisfying that $z_{i}$ converges to $z_{\infty}$, there exists $r>0$ such that

$$
\limsup _{i \rightarrow \infty}\left|\frac{1}{\underline{\operatorname{vol}} B_{t}\left(x_{i}\right)} \int_{B_{t}\left(x_{i}\right)}\left\langle d r_{z_{i}}, d f_{i}\right\rangle d \underline{\mathrm{vol}}-\frac{1}{v\left(B_{t}\left(x_{\infty}\right)\right)} \int_{B_{t}\left(x_{\infty}\right)}\left\langle d r_{z_{\infty}}, d f_{\infty}\right\rangle d v\right|<\epsilon
$$

and

$$
\limsup _{i \rightarrow \infty} \frac{1}{\underline{\operatorname{vol}} B_{t}\left(x_{i}\right)} \int_{B_{t}\left(x_{i}\right)}\left|d f_{i}\right|^{2} d \underline{\mathrm{vol}} \leq \frac{1}{v\left(B_{t}\left(x_{\infty}\right)\right)} \int_{B_{t}\left(x_{\infty}\right)}\left|d f_{\infty}\right|^{2} d v+\epsilon
$$

for every $0<t<r$. Here $r_{z_{i}}$ is the distance function from $z_{i}: r_{z_{i}}(w)=\overline{z_{i}, w}$.

Roughly speaking, this convergence: $d f_{i} \rightarrow d f_{\infty}$, implies " $H_{1,2}$ (or $H_{1, p}$ )convergence with respect to the measured Gromov-Hausdorff topology". See Theorem 1.1 and Remark 4.5. If $d f_{i}$ converges to $d f_{\infty}$ on $B_{R}(y)$, then we denote it by $\left(f_{i}, d f_{i}\right) \rightarrow\left(f_{\infty}, d f_{\infty}\right)$ on $B_{R}(y)$. Assume $\left(f_{i}, d f_{i}\right) \rightarrow\left(f_{\infty}, d f_{\infty}\right)$ and $\left(g_{i}, d g_{i}\right) \rightarrow\left(g_{\infty}, d g_{\infty}\right)$ on $B_{R}(y)$ below.

In the paper, we will study several properties of the convergence and give their applications. For example, we will show the following in Section 4:

Theorem 1.1. Let $\left\{F_{i}\right\}_{1 \leq i \leq \infty}$ be a sequence of continuous functions on R. Assume that $F_{i}$ converges to $F_{\infty}$ with respect to the compact uniformly topology. Then, we have

$$
\lim _{i \rightarrow \infty} \int_{B_{R}\left(m_{i}\right)} F_{i}\left(\left\langle d f_{i}, d g_{i}\right\rangle\right) d \underline{\mathrm{vol}}=\int_{B_{R}(y)} F_{\infty}\left(\left\langle d f_{\infty}, d g_{\infty}\right\rangle\right) d v .
$$

Especially, if $f_{\infty}=g_{\infty}$, then

$$
\lim _{i \rightarrow \infty} \int_{B_{R}\left(m_{i}\right)} F_{i}\left(\left|d f_{i}-d g_{i}\right|\right) d \underline{\mathrm{vol}}=F_{\infty}(0) v\left(B_{R}(y)\right) .
$$

See Corollary 4.4 for the proof. We will also show the following in Section 4: 
Theorem 1.2. Let $\left\{h_{i}\right\}_{1 \leq i<\infty}$ be a sequence of harmonic functions $h_{i}$ on $B_{R}\left(m_{i}\right)$, and $h_{\infty}$ a Lipschitz function on $B_{R}(y)$. Assume that $\sup _{i} \operatorname{Lip} h_{i}$ $<\infty$ and that $h_{i}$ converges to $h_{\infty}$ on $B_{R}(y)$. Then we have $\left(h_{i}, d h_{i}\right) \rightarrow$ $\left(h_{\infty}, d h_{\infty}\right)$ on $B_{R}(y)$.

We remark that in Theorem $1.2, h_{\infty}$ is a harmonic function on $B_{R}(y)$, proved in [11] by Ding. We will give an alternative proof of it in Section 4. See Corollary 4.7.

The organization of this paper is as follows:

In the next section, we will recall several important notions and propeties of metric spaces, Riemannian manifolds and their limit spaces. Most of statements in Section 2 do not have the proof, we will give a reference for them only.

In Section 3, we will show several results about rectifiability of limit spaces of Riemannian manifolds. See Theorems 3.1 and 3.4. It is important that their functions in these theorems which give a rectifiability of limit spaces, are distance functions. As a corollary of them, we will give an explicit geometric formula for the radial derivative of Lipschitz functions from a given point. See Theorem 3.3. These results are used in Section 4 essentially.

In Section 4, we will give two-definitions of pointwise convergence of $L^{\infty}$-functions with respect to the measured Gromov-Hausdorff topology, and give the definition of a convergence of the differentials of Lipschitz functions again via the definitions of convergence of $L^{\infty}$-functions. We will also give several properties of the convergence. The main properties are Theorems 4.1, 4.2 and Corollary 4.5.

Finally, we shall introduce several applications of this paper. In [24], we will give an application of this Section 4 to a study of harmonic functions with polynomial growth on asymptotic cones of non-negatively Riccicurved manifolds having Euclidean volume growth. For example, we will show that a space of harmonic functions on asymptotic cones with polynomial growth of a fixed rate is a finite-dimensional vector space. We can regard it as asymptotic cones version of the conjecture [9, Conjecture 0.1] by Yau $[39,40]$. Moreover, in [24], we will give "Laplacian comparison theorems on limit spaces of Riemannian manifolds" by using several results given in Section 4, and show a stability of lower bounds on Ricci curavture with respect to the Gromov-Hausdorff topology as a corollary of them. In [25], we will also give a geometric application by using several results in this Section 4, to limit spaces of Riemannian manifolds with Ricci curvature bounded below. 


\section{Background}

Our aim in this section is to give several notation, important notions and properties for metric measure spaces and manifolds. For a positive number $\epsilon>0$ and real numbers $a, b$, we use the following notations:

$$
a=b \pm \epsilon \Longleftrightarrow|a-b|<\epsilon
$$

We denote by $\Psi\left(\epsilon_{1}, \epsilon_{2}, \ldots, \epsilon_{k} ; c_{1}, c_{2}, \ldots, c_{l}\right)$ (more simply, $\Psi$ ) some positive function on $\mathbf{R}_{>0}^{k} \times \mathbf{R}^{l}$ satisfying

$$
\lim _{\epsilon_{1}, \epsilon_{2}, \ldots, \epsilon_{k} \rightarrow 0} \Psi\left(\epsilon_{1}, \epsilon_{2}, \ldots, \epsilon_{k} ; c_{1}, c_{2}, \ldots, c_{l}\right)=0
$$

for each fixed real numbers $c_{1}, c_{2}, \ldots, c_{l}$. We often denote by $C\left(c_{1}, c_{2}, \ldots, c_{l}\right)$ some positive constant depending only on fixed real numbers $c_{1}, c_{2}, \ldots, c_{l}$.

\subsection{Metric measure spaces}

For a metric space $Z$, a point $z \in Z$ and positive numbers $r, R$ with $r<R$, we use the following notations: $B_{r}(z)=\{x \in Z ; \overline{z, x}<r\}, \bar{B}_{r}(z)=\{x \in Z$; $\overline{z, x} \leq r\}, \partial B_{r}(z)=\{x \in Z ; \overline{z, x}=r\}$. Here $\overline{y, x}$ is the distance between $y$ and $x$, we often denote the distance by $d_{Z}(y, x)$. For every subset $A$ of $Z$, we also put $B_{r}(A)=\{x \in Z ; \overline{A, w}<r\}$ and $\bar{B}_{r}(A)=\{x \in Z ; \overline{A, x} \leq r\}$. For $z \in Z$, we define a 1-Lipschitz function $r_{z}$ on $Z$ by $r_{z}(w)=\overline{z, w}$. For a Lipschitz function $f$ on $Z$ and a point $z \in Z$, which is not isolated in $Z$, we put

$$
\begin{gathered}
\operatorname{lip} f(z)=\liminf _{r \rightarrow 0}\left(\sup _{x \in B_{r}(z) \backslash\{z\}} \frac{|f(x)-f(z)|}{\overline{x, z}}\right), \\
\operatorname{Lip} f(z)=\limsup _{r \rightarrow 0}\left(\sup _{x \in B_{r}(z) \backslash\{z\}} \frac{|f(x)-f(z)|}{\overline{x, z}}\right) .
\end{gathered}
$$

If $z$ is an isolated point in $Z$, then we put $\operatorname{lip} f(z)=\operatorname{Lip} f(z)=0$. We also denote the Lipschitz constant of $f$ by $\operatorname{Lip} f$. We remark that for every subset $A$ of $Z$ and every Lipschitz function $f$ on $A$, there exists a Lipschitz function $f^{*}$ on $Z$ such that $\left.f^{*}\right|_{A}=f$ and $\operatorname{Lip} f^{*}=\operatorname{Lip} f$. See for instance (8.2) in [2].

We say that $Z$ is proper if every bounded subset of $Z$ is relatively compact. We also say that $Z$ is a geodesic space if for every $x_{1}, x_{2} \in Z$, there exists an isometric embedding $\gamma$ from $\left[0, \overline{x_{1}, x_{2}}\right]$ to $Z$ such that $\gamma(0)=$ 
$x_{1}, \gamma\left(\overline{x_{1}, x_{2}}\right)=x_{2} \cdot \gamma$ is called a minimal geodesic from $x_{1}$ to $x_{2}$. For a proper geodesic space $W$ and a point $w$ in $W$, we put $C_{w}=\{z \in W ; \overline{w, z}+\overline{z, x}>$ $\overline{w, x}$ for every $x \in W \backslash\{z\}\}$ (if $W$ is a single point, then we put $C_{w}=\emptyset$ ), and call it the cut locus of $W$ at $w$.

For a proper metric space $Z$ and a Radon measure $v$ on $Z$, we say that the pair $(Z, v)$ is a metric measure space in this paper. For a metric measure space $(Z, v)$, a point $z$ in $Z$ and a non-negative integer $k$, we say that $v$ is Ahlfors $k$-regular at $z$ if there exist $r>0$ and $C \geq 1$ such that $C^{-1} \leq v\left(B_{t}(z)\right) / t^{k} \leq C$ for every $0<t<r$. We shall introduce the notion of $v$-rectifiability for metric measure spaces by Cheeger-Colding. See [6, Definition 5.3] and [6, Theorem 5.7]. See also [12]. For metric spaces $X_{1}, X_{2}$, a positive number $\delta$ with $\delta<1$, and a bijection map $f$ from $X_{1}$ to $X_{2}$, we say that $f$ is $(1 \pm \delta)$-bi-Lipschitz to $X_{2}$ if $f$ and $f^{-1}$ are $(1+\delta)$-Lipschitz maps.

Definition 2.1 Rectifiability for a Borel subset of metric measure spaces. For a metric measure space $(Z, v)$ and a Borel subset $A$ of $Z$, we say that $A$ is v-rectifiable if there exists a positive integer $m$, a collection of Borel subsets $\left\{C_{k, i}\right\}_{1 \leq k \leq m, i \in \mathbf{N}}$ of $A$, and a collection of bi-Lipschitz embedding maps $\left\{\phi_{k, i}: C_{k, i} \rightarrow \mathbf{R}^{k}\right\}_{k, i}$ such that the following properties hold:

1. $v\left(A \backslash \bigcup_{k, i} C_{k, i}\right)=0$

2. $v$ is Ahlfors $k$-regular at each $x \in C_{k, i}$.

3. For every $k, x \in \bigcup_{i \in \mathbf{N}} C_{k, i}$ and every $0<\delta<1$, there exists $C_{k, i}$ such that $x \in C_{k, i}$ and that the map $\phi_{k, i}$ is $(1 \pm \delta)$-bi-Lipschitz to the image $\phi_{k, i}\left(C_{k, i}\right)$.

Remark 2.1. The third ( $1 \pm \delta)$-bi-Lipschitz condition in the above definition is important. Actually, the existence of the canonical inner product of the cotangent bundle of Ricci limit spaces follows from this property. See condition (iii) of page 60 of [6] and Section 6 in [6].

\subsection{Gromov-Hausdorff convergence}

For compact metric spaces $X_{1}, X_{2}$, we denote the Gromov-Hausdorff distance between $X_{1}$ and $X_{2}$ by $d_{\mathrm{GH}}\left(X_{1}, X_{2}\right)$. See [17] for the definition. On the other hand, for compact metric spaces $X_{1}, X_{2}$, a positive number $\epsilon>0$ and a map $\phi$ from $X_{1}$ to $X_{2}$, we say that $\phi$ is an $\epsilon$-Gromov-Hausdorff approximation if $X_{2}=B_{\epsilon}($ Image $\phi)$ and $|\overline{x, y}-\overline{\phi(x), \phi(y)}|<\epsilon$ for every $x, y \in X_{1}$. For a sequence of compact metric spaces $\left\{X_{i}\right\}_{1 \leq i \leq \infty}$, we say that $X_{i}$ converges to 
$X_{\infty}$ if $d_{\mathrm{GH}}\left(X_{i}, X_{\infty}\right)$ converges to 0 . Then we denote it by $X_{i} \rightarrow X_{\infty}$. Similarly, for pointed compact metric spaces $\left(X_{1}, x_{1}\right),\left(X_{2}, x_{2}\right)$, we can define the pointed Gromov-Hausdorff distance $d_{\mathrm{GH}}\left(\left(X_{1}, x_{1}\right),\left(X_{2}, x_{2}\right)\right)$. Moreover, for a sequence of pointed proper geodesic spaces $\left\{\left(Z_{i}, z_{i}\right)\right\}_{1 \leq i \leq \infty}$, we say that $\left(Z_{i}, z_{i}\right)$ converges to $\left(Z_{\infty}, z_{\infty}\right)$ if there exist sequences $\left\{\epsilon_{i}\right\}_{i},\left\{R_{i}\right\}_{i}$ of positive numbers, and $\left\{\phi_{i}\right\}_{i}$ of Borel maps $\phi_{i}$ from $\left(B_{R_{i}}\left(z_{i}\right), z_{i}\right)$ to $\left(B_{R_{i}}\left(z_{\infty}\right), z_{\infty}\right)$ such that $\epsilon_{i} \rightarrow 0, R_{i} \rightarrow \infty$ as $i \rightarrow \infty, B_{R_{i}}\left(z_{\infty}\right) \subset B_{\epsilon_{i}}\left(\right.$ Image $\left.\phi_{i}\right)$ and $\mid \overline{\alpha, \beta}-$ $\overline{\phi_{i}(\alpha), \phi_{i}(\beta)} \mid \leq \epsilon_{i}$ for every $\alpha, \beta \in B_{R_{i}}\left(x_{i}\right)$. We denote it by $\left(Z_{i}, z_{i}\right) \stackrel{\left(\phi_{1}, R_{i}, \epsilon_{i}\right)}{\longrightarrow}$ $\left(Z_{\infty}, z_{\infty}\right)$, or more simply, $\left(Z_{i}, z_{i}\right) \rightarrow\left(Z_{\infty}, z_{\infty}\right)$. It is easy to check that $\left(Z_{i}, z_{i}\right) \rightarrow\left(Z_{\infty}, z_{\infty}\right)$ if and only if $d_{\mathrm{GH}}\left(\left(\bar{B}_{R}\left(z_{i}\right), z_{i}\right),\left(\bar{B}_{R}\left(z_{\infty}\right), z_{\infty}\right)\right) \rightarrow 0$ for every $R>0$. For a sequence $\left\{x_{i}\right\}_{1 \leq i \leq \infty}$ of points $x_{i} \in Z_{i}$, we say that $x_{i}$ converges to $x_{\infty}$ if $x_{i} \in B_{R_{i}}\left(z_{i}\right)$ and $\overline{\phi_{i}\left(x_{i}\right), x_{\infty}} \rightarrow 0$. Then, we denote it by $x_{i} \rightarrow x_{\infty}$.

Let $\left(Z_{i}, z_{i}\right) \rightarrow\left(Z_{\infty}, z_{\infty}\right)$. For a sequence $\left\{A_{i}\right\}_{1 \leq i \leq \infty}$ of subsets $A_{i}$ of $Z_{i}$ with $\sup _{i} \overline{z_{i}, A_{i}}<\infty$, we say that $A_{i}$ is included by $A_{\infty}$ asymptotically if for every $\epsilon>0$, there exists $i_{0}$ such that $\phi_{i}\left(A_{i}\right) \subset B_{\epsilon}\left(A_{\infty}\right)$ for every $i \geq i_{0}$. Then we denote it by $\limsup \sup _{i \rightarrow \infty}^{\mathrm{GH}} A_{i} \subset A_{\infty}$ (if $A_{\infty}=\emptyset$, then $\limsup _{i \rightarrow \infty}^{\mathrm{GH}} A_{i} \subset$ $A_{\infty}$ implies $A_{i}=\emptyset$ for every sufficiently large $i$ ). Similarly, we also say that $A_{\infty}$ is included by $A_{i}$ asymptotically if for every $\epsilon>0$, there exists $i_{0}$ such that $A_{\infty} \subset B_{\epsilon}\left(\phi_{i}\left(A_{i}\right)\right)$ for every $i \geq i_{0}$. Then we denote it by $A_{\infty} \subset$ $\liminf \operatorname{in}_{i \rightarrow \infty}^{\mathrm{GH}} A_{i}$. Let $C_{\infty} \subset \liminf _{i \rightarrow \infty}^{\mathrm{GH}} C_{i}$. For a sequence $\left\{f_{i}\right\}_{1 \leq i \leq \infty}$ of Lipschitz functions $f_{i}$ on $C_{i}$ with $\sup _{i} \operatorname{Lip} f_{i}<\infty$, we say that $f_{\infty}$ is a restriction of $f_{i}$ asymptotically if $\lim _{i \rightarrow \infty} f_{n(i)}\left(w_{n(i)}\right)=f_{\infty}(w)$ for every $w \in C_{\infty}$, every subsequence $\{n(i)\}_{i}$ of $\mathbf{N}$, and every $w_{n(i)} \in C_{n(i)}$ with $\overline{\phi_{n(i)}\left(w_{n(i)}\right), w} \rightarrow 0$. Let $\limsup _{i \rightarrow \infty} D_{i} \subset D_{\infty}$ and assume that $D_{\infty}$ is compact. For a sequence $\left\{g_{i}\right\}_{1 \leq i \leq \infty}$ of Lipschitz function $g_{i}$ on $D_{i}$ with $\sup _{i} \operatorname{Lip} g_{i}<\infty$, we say that $g_{\infty}$ is an extension of $g_{i}$ asymptotically if $\lim _{i \rightarrow \infty} g_{n(i)}\left(w_{n(i)}\right)=g_{\infty}(w)$ for every $w \in D_{\infty}$, every subsequence $\{n(i)\}_{i}$ of $\mathbf{N}$, and every $w_{n(i)} \in D_{n(i)}$ with $\overline{\phi_{n(i)}\left(w_{n(i)}\right), w} \rightarrow 0$.

For a sequence $\left\{K_{i}\right\}_{1 \leq i \leq \infty}$ of compact subsets $K_{i}$ of $Z_{i}$, we say that $\left(Z_{i}, z_{i}, K_{i}\right)$ converges to $\left(Z_{\infty}, z_{\infty}, K_{\infty}\right)$ if $\limsup _{i \rightarrow \infty}^{\mathrm{GH}} K_{i} \subset K_{\infty}$ and $K_{\infty} \subset$ $\lim \inf _{i \rightarrow \infty}^{\mathrm{GH}} K_{i}$ hold. Then we denote it by $\left(Z_{i}, z_{i}, K_{i}\right) \stackrel{\left(\phi_{i}, R_{i}, \epsilon_{i}\right)}{\longrightarrow}\left(Z_{\infty}, z_{\infty}, K_{\infty}\right)$, or more simply, $\left(Z_{i}, z_{i}, K_{i}\right) \rightarrow\left(Z_{\infty}, z_{\infty}, K_{\infty}\right)$, or $K_{i} \rightarrow K_{\infty}$.

Let $\left(Z_{i}, z_{i}, K_{i}\right) \rightarrow\left(Z_{\infty}, z_{\infty}, K_{\infty}\right)$. For sequences $\left\{f_{i}^{1}\right\}_{1 \leq i \leq \infty}, \ldots$, $\left\{f_{i}^{k}\right\}_{1 \leq i \leq \infty}$ of Lipschitz functions $f_{i}^{l}$ on $K_{i}$ with $\sup _{i, l}\left(\operatorname{Lip} f_{i}^{l}+\left|f_{i}^{l}\right|_{L^{\infty}}\right)<\infty$, we say that $\left(Z_{i}, z_{i}, K_{i}, f_{i}^{1}, \ldots, f_{i}^{k}\right)$ converges to $\left(Z_{\infty}, z_{\infty}, K_{\infty}, f_{\infty}^{1}, \ldots, f_{\infty}^{k}\right)$ if $f_{\infty}^{l}$ is an extension of $\left\{f_{i}^{l}\right\}_{i}$ asymptotically for every $l$. We denote it by $\left(Z_{i}, z_{i}, K_{i}, f_{i}^{1}, \ldots, f_{i}^{k}\right) \rightarrow\left(Z_{\infty}, z_{\infty}, K_{\infty}, f_{\infty}^{1}, \ldots, f_{\infty}^{k}\right)$, or more simply, $f_{i}^{l} \rightarrow$ $f_{\infty}^{l}$ for every $l$. Then it is easy to check that $\lim _{i \rightarrow \infty}\left|f_{i}^{l}-f_{\infty}^{l} \circ \phi_{i}\right|_{L^{\infty}\left(K_{i}\right)}=0$. 
It is not difficult to check the following proposition:

Proposition 2.1. Let $\left\{\left(Z_{i}, z_{i}\right)\right\}_{1 \leq i \leq \infty}$ be a sequence of pointed proper geodesic spaces, $\Lambda$ a set and $\left\{A_{i}^{\lambda}\right\}_{\lambda \in \Lambda}$ a collection of bounded subsets of $Z_{i}$ for every $1 \leq i \leq \infty$. Assume that $\left(Z_{i}, z_{i}\right)$ converges to $\left(Z_{\infty}, z_{\infty}\right), A_{\infty}^{\lambda}$ is compact for every $\lambda \in \Lambda$ and that $\limsup _{i \rightarrow \infty}^{\mathrm{GH}} A_{i}^{\lambda} \subset A_{\infty}^{\lambda}$ for every $\lambda \in \Lambda$. Then, we have $\lim \sup _{i \rightarrow \infty}^{\mathrm{GH}} \bigcap_{\lambda \in \Lambda} A_{i}^{\lambda} \subset \bigcap_{\lambda \in \Lambda} A_{\infty}^{\lambda}$ and $\limsup _{i \rightarrow \infty}^{\mathrm{GH}}\left(A_{i} \backslash B_{r}\left(x_{i}\right)\right) \subset$ $A_{\infty} \backslash B_{r}\left(x_{\infty}\right)$ for every $r>0$ and every sequence $\left\{x_{i}\right\}_{i}$ of points $x_{i}$ in $Z_{i}$ with $x_{i} \rightarrow x_{\infty}$.

We shall recall a fundamental covering lemma for proper metric spaces. See Chapter 1 in [38] for the proof.

Proposition 2.2. Let $X$ be a proper metric space, $A$ a subset of $X, \Lambda$ a set, $\left\{x_{\lambda}\right\}_{\lambda \in \Lambda}$ a collection of points in $X$ and $\left\{r_{\lambda}\right\}_{\lambda \in \Lambda}$ a collection of positive numbers. Assume that for every $x \in A$ and every $\epsilon>0$, there exists $\lambda \in \Lambda$ such that $x \in \bar{B}_{r_{\lambda}}\left(x_{\lambda}\right)$ and $\operatorname{diam} \bar{B}_{r_{\lambda}}\left(x_{\lambda}\right)<\epsilon$. Then, there exists a countable subset $\Lambda_{1}$ of $\Lambda$ such that the following properties hold:

1. $\left\{\bar{B}_{r_{\lambda_{1}}}\left(x_{\lambda_{1}}\right)\right\}_{\lambda_{1} \in \Lambda_{1}}$ are pairwise disjoint collection.

2. We have

$$
A \backslash \bigcup_{\lambda_{2} \in \Lambda_{2}} \bar{B}_{r_{\lambda_{2}}}\left(x_{\lambda_{2}}\right) \subset \bigcup_{\lambda \in \Lambda_{1} \backslash \Lambda_{2}} \bar{B}_{5 r_{\lambda}}\left(x_{\lambda}\right)
$$

for every finite subset $\Lambda_{2}$ of $\Lambda_{1}$.

We shall recall the definition of measured Gromov-Hausdorff convergence. Let $\left(Z_{i}, z_{i}\right) \rightarrow\left(Z_{\infty}, z_{\infty}\right)$. For a sequence $\left\{v_{i}\right\}_{1 \leq i \leq \infty}$ of Radon measures $v_{i}$ on $Z_{i}$, we say that $\left(Z_{i}, z_{i}, v_{i}\right)$ converges to $\left(Z_{\infty}, z_{\infty}, v_{\infty}\right)$ with respect to the measured Gromov-Hausdorff topology if $\lim _{i \rightarrow \infty} v_{i}\left(B_{r}\left(x_{i}\right)\right)=$ $v_{\infty}\left(B_{r}\left(x_{\infty}\right)\right)$ for every $r>0$ and every sequence $\left\{x_{i}\right\}_{i}$ of points $x_{i}$ in $Z_{i}$ with $x_{i} \rightarrow x_{\infty}$. See also [13]. Then we denote it by $\left(Z_{i}, z_{i}, v_{i}\right) \rightarrow\left(Z_{\infty}, z_{\infty}, v_{\infty}\right)$. The next proposition is used many times in this paper. We skip the proof because it is not difficult to check it by using Proposition 2.2.

Proposition 2.3. Let $\left\{\left(Z_{i}, z_{i}, v_{i}\right)\right\}_{1 \leq i \leq \infty}$ be a sequence of pointed proper geodesic spaces with Radon measures, and $\left\{A_{i}\right\}_{1 \leq i \leq \infty}$ a sequence of Borel subsets $A_{i}$ of $Z_{i}$. Assume that $v_{i}\left(B_{1}\left(z_{i}\right)\right)=1, A_{\infty}$ is compact, $\left(Z_{i}, z_{i}, v_{i}\right) \rightarrow$ $\left(Z_{\infty}, z_{\infty}, v_{\infty}\right)$, $\limsup _{i \rightarrow \infty}^{G H} A_{i} \subset A_{\infty}$ and that for every $R>0$ there exists 
$\kappa=\kappa(R) \geq 1$ such that $v_{i}\left(B_{2 r}\left(x_{i}\right)\right) \leq 2^{\kappa} v_{i}\left(B_{r}\left(x_{i}\right)\right)$ for every $0<r<R$, every $1 \leq i \leq \infty$ and every $x_{i} \in Z_{i}$. Then we have

$$
\limsup _{i \rightarrow \infty} v_{i}\left(A_{i}\right) \leq v_{\infty}\left(A_{\infty}\right)
$$

We shall give a proof of the following proposition:

Proposition 2.4. Let $\left\{\left(Z_{i}, z_{i}, v_{i}\right)\right\}_{1 \leq i \leq \infty}$ be a sequence of pointed proper geodesic spaces with Radon measures. Assume that $v_{i}\left(B_{1}\left(z_{i}\right)\right)=1$ for every $i$, $\operatorname{diam} Z_{\infty}>0,\left(Z_{i}, z_{i}, v_{i}\right) \stackrel{\left(\phi_{i}, R_{i}, \epsilon_{i}\right)}{\longrightarrow}\left(Z_{\infty}, z_{\infty}, v_{\infty}\right)$, and that for every $R>0$, there exists $\kappa=\kappa(R) \geq 1$ such that $v_{i}\left(B_{2 r}\left(x_{i}\right)\right) \leq 2{ }^{\kappa} v_{i}\left(B_{r}\left(x_{i}\right)\right)$ for every $0<r<R$, every $1 \leq i \leq \infty$ and every $x_{i} \in Z_{i}$. Then, we have

$$
\lim _{i \rightarrow \infty} \sup _{x_{i} \in B_{R}\left(z_{i}\right), 0<r<R}\left|v_{i}\left(B_{r}\left(x_{i}\right)\right)-v_{\infty}\left(B_{r}\left(\phi_{i}\left(x_{i}\right)\right)\right)\right|=0
$$

for every $R \geq 1$.

Proof. It is easy to check that $\operatorname{rad} Z_{\infty}>0$. Here $\operatorname{rad} X=\inf _{x_{2} \in X}\left(\sup _{x_{1} \in X}\right.$ $\left.\overline{x_{1}, x_{2}}\right)$ for a metric space $X$. Put $\kappa=\kappa(100 R)$. Let $\tau>0$ with $\tau \ll \operatorname{rad} Z_{\infty}$. Then, there exists $N$ such that for every $N \leq i \leq \infty$ and every $w \in Z_{i}$, there exists $\hat{w} \in Z_{i}$ such that $\overline{w, \hat{w}}=\tau$. Since $B_{\delta}(w) \subset B_{\tau+\delta}(\hat{w}) \backslash B_{\tau-\delta}(\hat{w})$ for every $0<\delta<\tau$, by [10, Lemma 3.3], there exists $\hat{\tau} \ll \tau$ such that $v_{i}\left(B_{t}(w)\right)$ $\leq \Psi(t ; \kappa, R) v_{i}\left(B_{10 \tau}(w)\right)$ for every $N \leq i \leq \infty$, every $w \in Z_{i}$ and every $0<$ $t<\hat{\tau}$. Fix $\epsilon>0$. Then, there exist $N_{1} \in \mathbf{N}$ and $0<r_{1} \ll \min \{R, \hat{\tau}, \epsilon, 1\}$ such that $v_{i}\left(B_{s}(z)\right) \leq \epsilon$ for every $N_{1} \leq i \leq \infty$, every $0<s<r_{1}$ and every $z \in B_{R}\left(z_{i}\right)$. Let $\left\{x_{j}\right\}_{1 \leq j \leq l} \subset B_{R}\left(z_{\infty}\right)$ and $\left\{t_{j}\right\}_{1 \leq j \leq \hat{l}} \subset[0, R]$ satisfying that $B_{R}\left(z_{\infty}\right) \subset \bigcup_{j=1}^{l} B_{\epsilon r_{1}}\left(x_{j}\right)$ and $[0, R] \subset \bigcup_{j=1}^{\hat{l}} B_{\epsilon r_{1}}\left(t_{j}\right)$. Let $x_{j}(i) \in B_{R}\left(z_{i}\right)$ with $x_{j}(i) \rightarrow x_{j}$. There exists $N_{2} \geq N_{1}$ such that $\left|v_{i}\left(B_{t_{\hat{j}}}\left(x_{j}(i)\right)\right)-v_{\infty}\left(B_{t_{\hat{j}}}\left(x_{j}\right)\right)\right|<$ $\epsilon$ for every $i \geq N_{2}$, every $1 \leq j \leq l$ and every $1 \leq \hat{j} \leq \hat{l}$. Fix $z \in B_{R}\left(z_{\infty}\right)$ and $s \in\left[r_{1}, R\right]$. Let $j \in\{1, \ldots, l\}$ and $\hat{j} \in\{1, \ldots, \hat{l}\}$ satisfying that $\overline{z, x_{j}}<$ $\epsilon r_{1}$ and $\left|s-t_{\hat{j}}\right|<\epsilon r_{1}$. Then, by [10, Lemma 3.3], we have $\mid v_{\infty}\left(B_{s}(z)\right)-$ $v_{\infty}\left(B_{t_{\hat{j}}}\left(x_{j}\right)\right) \mid \leq v_{\infty}\left(B_{s+5 \epsilon r_{1}}(z)\right)-v_{\infty}\left(B_{s-5 \epsilon r_{1}}(z)\right) \leq \Psi(\epsilon ; \kappa, R, \tau) v_{\infty}\left(B_{R}\left(z_{\infty}\right)\right)$ On the other hand, for a sequence $\{z(i)\}_{i}$ of points $z(i)$ in $B_{R}\left(z_{i}\right)$ with $z(i) \rightarrow$ $z,\left|v_{i}\left(B_{s}(z(i))\right)-v_{i}\left(B_{t_{\hat{j}}}\left(x_{j}(i)\right)\right)\right| \leq v_{i}\left(B_{s+10 \epsilon r_{1}}(z(i))\right)-v_{i}\left(B_{s-10 \epsilon r_{1}}(z(i))\right) \leq$ $\Psi(\epsilon ; \kappa, R, \tau) v_{i}\left(B_{R}\left(z_{i}\right)\right) \leq \Psi(\epsilon ; \kappa, R, \tau) v_{\infty}\left(B_{R}\left(z_{\infty}\right)\right)$ for every $i \geq N_{2}$. Thus, we have $\left|v_{i}\left(B_{s}(z(i))\right)-v_{\infty}\left(B_{s}(z)\right)\right|<\Psi(\epsilon ; \kappa, R, \tau) v_{\infty}\left(B_{R}\left(z_{\infty}\right)\right)$ for every $i \geq$ $N_{2}$. Therefore, we have the assertion. 


\subsection{Riemannian manifolds and their limit spaces}

For a real number $K$ and a pointed proper geodesic space $(Y, y)$, in this paper, we say that $(Y, y)$ is a $(n, K)$-Ricci limit space if there exist sequences of real numbers $\left\{K_{i}\right\}_{i}$, and of pointed $n$-dimensional complete Riemannian manifolds $\left\{\left(M_{i}, m_{i}\right)\right\}_{i}$ with $\operatorname{Ric}_{M_{i}} \geq K_{i}(n-1)$ such that $K_{i} \rightarrow K$ and $\left(M_{i}, m_{i}\right) \rightarrow(Y, y)$. Similarly, for a pointed proper geodesic space with Radon measure $(Y, y, v)$, we also say that $(Y, y, v)$ is a $(n, K)$-Ricci limit space $\left(\right.$ of $\left.\left\{\left(M_{i}, m_{i}, \mathrm{vol}\right)\right\}_{i}\right)$ if $\left(M_{i}, m_{i}, \mathrm{vol}\right) \rightarrow(Y, y, v)$ as above. More simply, for a $(n,-1)$-Ricci limit space $(Y, y)$ (or $(Y, y, v)$ ), we say that $(Y, y)$ is a Ricci limit space. See for instance Section 4.1 in [34]. We shall fix a Ricci limit space $(Y, y, v)$ in this subsection and give a very short review of structure theory of Ricci limit spaces developed by Cheeger-Colding, Colding, below. See $[3-6,8]$ for the details.

For pointed proper geodesic spaces $(Z, z)$ and $(X, x)$, we say that $(Z, z)$ is a tangent cone of $X$ at $x$ if there exists a sequence of positive numbers $\left\{r_{i}\right\}_{i}$ such that $r_{i} \rightarrow 0$ and $\left(X, x, r_{i}^{-1} d_{X}\right) \rightarrow(Z, z)$. For $k \geq 1$, we put $\mathcal{R}_{k}(Y)=\left\{x \in Y\right.$; All tangent cones at $x$ are isometric to $\left.\mathbf{R}^{k}\right\}$ and call it the $k$-dimensional regular set. More simply, we shall denote it by $\mathcal{R}_{k}$. We also put $\mathcal{R}=\bigcup_{1<k<n} \mathcal{R}_{k}$ and call it the regular set. Then we have $v(Y \backslash \mathcal{R})=0$. See $[4$, Theorem 2.1] for the proof. For $\delta, r>0$ and $0<$ $\alpha<1$, we put $\left(\mathcal{R}_{k}\right)_{\delta, r}=\left\{x \in Y ; d_{\mathrm{GH}}\left(\left(\bar{B}_{s}(x), x\right),\left(\bar{B}_{s}\left(0_{k}\right), 0_{k}\right)\right) \leq \delta s\right.$ for every $0<s \leq r\}$ and $\left(\mathcal{R}_{k ; \alpha}\right)_{r}=\left\{x \in Y ; d_{\mathrm{GH}}\left(\left(\bar{B}_{s}(x), x\right),\left(\bar{B}_{s}\left(0_{k}\right), 0_{k}\right)\right) \leq s^{1+\alpha}\right.$ for every $0<s \leq r\}$. Here $0_{k} \in \mathbf{R}^{k}$. We remark that $\left(\mathcal{R}_{k}\right)_{\delta, r}$ and $\left(\mathcal{R}_{k ; \alpha}\right)_{r}$ are closed, $\bigcap_{\delta>0}\left(\bigcup_{r>0}\left(\mathcal{R}_{k}\right)_{\delta, r}\right)=\mathcal{R}_{k}$. We also put $\mathcal{R}_{k ; \alpha}=\bigcup_{r>0}\left(\mathcal{R}_{k ; \alpha}\right)_{r}$. By $[4$, Theorem 3.23] and [4, Theorem 4.6], there exists $0<\alpha(n)<1$ such that $v\left(\mathcal{R}_{k} \backslash \mathcal{R}_{k ; \alpha(n)}\right)=0$ and that $v$ is Ahlfors $k$-regular at every point in $\mathcal{R}_{k ; \alpha(n)}$ for every $k$.

On the other hand, it is known that $Y$ is $v$-rectifiable. See [6, Theorem 5.5] and [6, Theorem 5.7]. Thus, by Section 6 in [6] or Section 4 in [2], the cotangent bundle $T^{*} Y$ of $Y$ exists. We will give several fundamental properties of the cotangent bundle only:

1. $T^{*} Y$ is a topological space.

2. There exists a Borel map $\pi: T^{*} Y \rightarrow Y$ such that $v\left(Y \backslash \pi\left(T^{*} Y\right)\right)=0$.

3. $\pi^{-1}(w)$ is a finite-dimensional real vector space with canonical inner product $\langle\cdot, \cdot\rangle(w)$ for every $w \in \pi\left(T^{*} Y\right)$.

4. For every open subset $U$ of $Y$ and every Lipschitz function $f$ on $U$, there exist a Borel subset $V$ of $U$, and a Borel map $d f$ (called 
the differential section of $f$ or the differential of $f$ ) from $V$ to $T^{*} Y$ such that $v(U \backslash V)=0$ and that $\pi \circ d f(w)=w,|d f|(w)=\operatorname{Lip} f(w)=$ $\operatorname{lip} f(w)$ for every $w \in V$, where $|v|(w)=\sqrt{\langle v, v\rangle(w)}$.

We call $\{\langle\cdot, \cdot\rangle(w)\}_{w \in \pi\left(T^{*} Y\right)}$ the Riemannian metric of $Y$ and denote it by $\langle\cdot, \cdot\rangle$. Finally, we remark that $v\left(C_{x}\right)=0$ for every $x \in Y$. See $[22$, Theorem 3.2]. These above results are used in Section 3, essentially.

\section{Rectifiability on limit spaces}

In this section, we shall study a rectifiability of Ricci limit spaces. These results given in this section are used in Section 4, essentially.

\subsection{Radial rectifiability}

The main result in this subsection is Theorem 3.1.

Lemma 3.1. Let $Z$ be a proper geodesic space, $z$ a point in $Z, s, \delta$ positive numbers, $v$ a Radon measure on $Z$ and $F$ a non-negative valued Borel function on $B_{s}(m)$. Assume that

$$
\frac{1}{v\left(B_{s}(z)\right)} \int_{B_{s}(z)} F d v \leq \delta
$$

and that there exists $\kappa \geq 1$ such that $0<v\left(B_{2 t}(w)\right) \leq 2 \kappa v\left(B_{t}(w)\right)$ for every $w \in B_{s}(z)$ and every $0<t \leq s$. Then, there exists a compact subset $K$ of $\bar{B}_{s / 10^{2}}(z)$ such that $v(K) / v\left(B_{s / 10^{2}}(z)\right) \geq 1-\Psi(\delta ; \kappa)$ and

$$
\frac{1}{v\left(B_{t}(x)\right)} \int_{B_{t}(x)} F d v \leq \Psi(\delta ; \kappa)
$$

for every $x \in K$ and every $0<t \leq s / 10^{2}$.

Proof. Without loss of generality, we can assume that $F$ is a non-negative valued Borel function on $Z$ by defining $F \equiv 0$ on $Z \backslash B_{s}(z)$. Fix $C>0$ and put $A_{1}(C)=\left\{w \in B_{s}(z) ; \int_{B_{s / 10^{2}}(w)} F d v \geq C v\left(B_{s / 10^{2}}(w)\right)\right\}$. Let $\left\{x_{j}^{1}\right\}_{1 \leq j \leq k_{1}}$ be an $s / 10$-maximal separated subset of $A_{1}(C)$. Put $A_{2}(C)=\left\{w \in B_{s}(m) \backslash\right.$ $\left.\bigcup_{i=1}^{k_{1}} B_{s}\left(x_{i}^{1}\right) ; \int_{B_{s / 10^{3}}(w)} F d v \geq C v\left(B_{s / 10^{3}}(w)\right)\right\}$. Let $\left\{x_{j}^{2}\right\}_{1 \leq j \leq k_{2}}$ be an $s / 10^{2}-$ maximal separated subset of $A_{2}(C)$. By iterating this argument, put $A_{l}(C)=\left\{w \in B_{s}(m) \backslash \bigcup_{1 \leq j \leq l-1,1 \leq i \leq k_{j}} B_{s / 10^{l-2}}\left(x_{i}^{l-1}\right) ; \int_{B_{s / 10^{l+1}}(w)} F d v \geq C v\right.$ 
$\left.\left(B_{s / 10^{l+1}}(w)\right)\right\}$. Let $\left\{x_{j}^{l}\right\}_{1 \leq j \leq k_{l}}$ be an $s / 10^{l}$-maximal separated subset of $A_{l}(C)$.

Claim 3.1. The collection $\left\{\bar{B}_{s / 10^{l+1}}\left(x_{i}^{l}\right)\right\}_{i, l}$ are pairwise disjoint.

Let $w \in \bar{B}_{s / 10^{\hat{\imath}+1}}\left(x_{\hat{i}}^{\hat{l}}\right) \cap \bar{B}_{s / 10^{l+1}}\left(x_{i}^{l}\right)$. Assume that $l<\hat{l}$. Then, by the construction, we have $x_{\hat{i}}^{\hat{l}} \in M \backslash \bigcup_{j=1}^{k_{l}} B_{s / 10^{l-1}}\left(x_{j}^{l}\right)$. Especially, we have $\overline{x_{\hat{i}}^{\hat{l}}, x_{i}^{l}} \geq$ $s / 10^{l-1}$. Therefore, we have $\bar{B}_{s / 10^{\hat{\imath}+1}}\left(x_{\hat{i}}^{\hat{l}}\right) \cap \bar{B}_{s / 10^{l+1}}\left(x_{i}^{l}\right)=\emptyset$. This is a contradiction. Therefore, we have $l=\hat{l}$. By the definition, we have $i=\hat{i}$. Thus, we have Claim 3.1.

It is easy to check the following claim:

Claim 3.2. We have $\bigcup_{i \in \mathbf{N}} A_{i}(C) \subset \bigcup_{l \in \mathbf{N}, 1 \leq i \leq k_{l}} \bar{B}_{s / 10^{l-2}}\left(x_{i}^{l}\right)$

We have

$$
\begin{aligned}
\sum_{l \in \mathbf{N}, 1 \leq i \leq k_{l}} \int_{B_{\frac{s}{10^{l+1}}}\left(x_{i}^{l}\right)} F d v \geq & C \sum_{l \in \mathbf{N}, 1 \leq i \leq k_{l}} v\left(B_{\frac{s}{10^{l+1}}}\left(x_{i}^{l}\right)\right) \\
\geq & C C(\kappa) \sum_{l \in \mathbf{N}, 1 \leq i \leq k_{l}} v\left(B_{\frac{s}{10^{l-2}}}\left(x_{i}^{l}\right)\right) \geq C C(\kappa) v \\
& \times\left(\bigcup_{l \in \mathbf{N}, 1 \leq i \leq k_{l}} B_{\frac{s}{10^{l-2}}}\left(x_{i}^{l}\right)\right) .
\end{aligned}
$$

On the other hand, we have

$$
\begin{aligned}
\sum_{l \in \mathbf{N}, 1 \leq i \leq k_{l}} \int_{B_{\frac{s}{10^{l+1}}}\left(x_{i}^{l}\right)} F d v & =\int_{\bigcup_{l \in \mathbf{N}, 1 \leq i \leq k_{l}} B_{\frac{s}{10^{l+1}}}\left(x_{i}^{l}\right)} F d v \leq \int_{B_{s}(z)} F d v \\
& \leq C(\kappa) v\left(B_{s}(z)\right) \delta .
\end{aligned}
$$

Therefore, we have

$$
\frac{v\left(\bigcup_{l \in \mathbf{N}, 1 \leq i \leq k_{l}} B_{\frac{s}{10^{l-2}}}\left(x_{i}^{l}\right)\right)}{v\left(B_{s}(m)\right)} \leq \frac{\delta}{C} C(\kappa) .
$$

By letting $C=\sqrt{\delta}$ and $K=\bar{B}_{s / 10^{2}}(z) \backslash \bigcup_{l \in \mathbf{N}, 1 \leq i \leq k_{l}} B_{\frac{s}{10^{l-2}}}\left(x_{i}^{l}\right)$, we have the assertion.

Let $(Y, y)$ be a Ricci limit space, $k$ an integer with $k \leq n$, and $r, \delta$ positive numbers with $r<1, \delta<1$. Let $\left(\mathcal{R}_{k}\right)_{\delta, r}^{y}$ be the set of points $w$ in $Y$ satisfying 
that for every $0<s \leq r$, there exists a map $\Phi$ from $\bar{B}_{s}(w)$ to $\mathbf{R}^{k}$ such that $\pi_{1} \circ \Phi=r_{y}$ and that $\Phi$ is a $\delta s$-Gromov-Hausdorff approximation to $\bar{B}_{s}(\Phi(w))$ Here, $\pi_{1}$ is the projection from $\mathbf{R}^{k}=\mathbf{R} \times \mathbf{R}^{k-1}$ to $\mathbf{R}$ defined by $\pi_{1}\left(x_{1}, \ldots, x_{k}\right)=x_{1}$.

Lemma 3.2. We have

$$
\bigcap_{\delta>0}\left(\bigcup_{r>0}\left(\left(\mathcal{R}_{k}\right)_{\delta, r}^{x} \backslash C_{x}\right)\right)=\mathcal{R}_{k} \backslash C_{x} .
$$

Proof. It is easy to check that

$$
\bigcap_{\delta>0}\left(\bigcup_{r>0}\left(\left(\mathcal{R}_{k}\right)_{\delta, r}^{x} \backslash C_{x}\right)\right) \subset \mathcal{R}_{k} \backslash C_{x} .
$$

Let $w \in \mathcal{R}_{k} \backslash C_{x}$. For every $\delta>0$, there exists $r>0$ such that for every $0<$ $s<r$, there exists a $\delta s$-Gromov-Hausdorff approximation from $\left(\bar{B}_{s}\left(0_{k}\right), 0_{k}\right)$ to $\left(\bar{B}_{s}(w), w\right)$. Here, $0_{k} \in \mathbf{R}^{k}$. On the other hand, by the splitting theorem on limit spaces [2, Theorem 9.27], there exist a pointed proper geodesic space $\left(W_{s}, w_{s}\right)$ and a map $\hat{\Phi}$ from $\left(\bar{B}_{s}(w), w\right)$ to $\left(\bar{B}_{s}\left(0, w_{s}\right),\left(0, w_{s}\right)\right)$ such that $\pi_{\mathbf{R}} \circ \hat{\Phi}=r_{x}-\overline{x, w}$ and that $\hat{\Phi}$ is a $\delta s$-Gromov-Hausdorff approximation. Here, $\bar{B}_{s}\left(0, w_{s}\right) \subset \mathbf{R} \times W_{s}$ with the product metric $\sqrt{d_{\mathbf{R}}^{2}+d_{W_{s}}^{2}}, \pi_{\mathbf{R}}$ is the projection from $\mathbf{R} \times W_{s}$ to $\mathbf{R}$. By rescaling $s^{-1} d_{\mathbf{R}^{k}}$ and [21, Claim 4.4], there exists a $\Psi(\delta ; n) s$-Gromov-Hausdorff approximation $f$ from $\left(\bar{B}_{s}\left(w_{s}\right), w_{s}\right)$ to $\left(\bar{B}_{s}\left(0_{k-1}\right), 0_{k-1}\right)$. Define a map $g$ from $\bar{B}_{s}(w)$ to $\mathbf{R}^{k}$ by $g(z)=(\overline{x, z}, f \circ \hat{\Phi})$. Let $\pi_{s}$ be the canonical retraction from $\mathbf{R}^{k}$ to $\bar{B}_{s}(g(w))$. Put $\hat{g}=\pi_{s} \circ g$. Then, it is easy to check that $\hat{g}$ is an $\Psi(\delta ; n) s$-Gromov-Hausdorff approximation to $\left(\bar{B}_{s}(\hat{g}(w)), \bar{g}(w)\right)$. Since $\delta$ is arbitrary, we have the assertion.

Put $\mathcal{D}_{x}^{\tau}=\{w \in X$; There exists $\alpha \in X$ such that $\overline{\alpha, w} \geq \tau$ and $\overline{x, w}+\overline{w, \alpha}=$ $\overline{x, \alpha}\}$ for a proper geodesic space $X$, a point $x \in X$ and a positive number $\tau>0$. It is easy to check that $\mathcal{D}_{x}^{\tau}$ is closed. By the definition, we have $\bigcup_{\tau>0} \mathcal{D}_{x}^{\tau}=X \backslash C_{x}$. Let Leb $A=\left\{a \in A ; \lim _{r \rightarrow 0} v\left(B_{r}(a) \cap A\right) / v\left(B_{r}(a)\right)=1\right\}$ for a metric measure space $(X, v)$ and a Borel subset $A$ of $X$.

We shall give a fundamental result about rectifiability of limit spaces by distance functions. The essential idea of the proof is to replace harmonic functions giving rectifiability in [6, Theorem 3.26] with suitable distance functions via the Poincaré inequality.

Lemma 3.3. Let $(Y, y, v)$ be a Ricci limit space, $k$ a positive integer satisfying $k \leq n, \delta, r$ positive numbers satisfying $\delta<1, r<1, x$ a point in $Y$ 
and $w$ a point in $\left(\mathcal{R}_{k}\right)_{\delta, r}^{x} \cap \operatorname{Leb}\left(\left(\mathcal{R}_{k}\right)_{\delta, r}\right) \backslash\left(C_{x} \cup\{x\}\right)$. Then, there exists $\eta(w)>0$ such that the following property holds: For every $0<s \leq \eta(w)$, there exists a compact subset $L$ of $\bar{B}_{s}(w) \cap\left(\mathcal{R}_{k}\right)_{\delta, r}$ and a collection of points $\left\{x_{j}\right\}_{2 \leq j \leq k}$ in $Y$ such that $v(L) / v\left(B_{s}(w)\right) \geq 1-\Psi(\delta ; n)$ and that the map $\Phi=\left(r_{x}, r_{x_{2}}, \ldots, r_{x_{k}}\right)$ from $L$ to $\mathbf{R}^{k}$, is an $(1 \pm \Psi(\delta ; n))$-bi-Lipschitz equivalent to the image $\Phi(L)$.

Proof. There exists $0<\tau<r$ such that $w \in \mathcal{D}_{x}^{\tau} \backslash B_{\tau}(x)$ and $v\left(B_{s}(w) \cap\right.$ $\left.\left(\mathcal{R}_{k}\right)_{\delta, r}\right) / v\left(B_{s}(w)\right) \geq 1-\delta$ for every $0<s<\tau$. Let $\left(M_{i}, m_{i}, \underline{\text { vol }}\right) \rightarrow(Y, y, v)$, and let $\left\{x_{i}\right\}_{i},\left\{w_{i}\right\}_{i}$ be sequences of points $x_{i}, w_{i}$ in $M_{i}$ satisfying that $w_{i} \rightarrow w$ and $x_{i} \rightarrow x$. Fix $0<s \ll \min \{\delta, \tau\}$. Then, for every sufficiently large $i$, there exists a $\delta s$-Gromov-Hausdorff approximation $\Phi^{i}=\left(\Phi_{1}^{i}, \ldots, \Phi_{k}^{i}\right)$ from $\left(\bar{B}_{s}\left(w_{i}\right), w_{i}\right)$ to $\left(\bar{B}_{s}\left(0_{k}\right), 0_{k}\right)$ such that $\Phi_{1}^{i}=r_{x_{i}}-r_{x_{i}}\left(w_{i}\right)$. Put $s_{0}=\sqrt{\delta} s$. For convenience, we shall use the following notations for rescaled metrics $s_{0}^{-1} d_{M_{i}}, s_{0}^{-1} d_{Y}: \hat{\mathrm{vol}}=\mathrm{vol}^{s_{0}^{-1} d_{M_{i}}}, \hat{r}_{w}(\alpha)=s_{0}^{-1} r_{w}(\alpha), \hat{B}_{t}(\alpha)=B_{t}^{s_{0}^{-1} d_{M_{i}}}(\alpha)=$ $B_{s_{0} t}(\alpha), \hat{v}=v / v\left(B_{s_{0}}(y)\right), \hat{g}=s_{0}^{-1} g$ for a Lipschitz function $g$ and so on. We also denote the differential section of $g$ as rescaled manifolds $\left(M_{i}, s_{0}^{-1} d_{M_{i}}\right)$ by $\hat{d} g: M_{i} \rightarrow T^{*} M_{i}$ and denote the Riemannian metric of $\left(M_{i}, s_{0}^{-1} d_{M_{i}}\right)$ by $\langle\cdot, \cdot\rangle_{s_{0}}=s_{0}^{-2}\langle\cdot, \cdot\rangle$. We remark that $\left(M_{i}, m_{i}, s_{0}^{-1} d_{M_{i}}, \underline{\mathrm{vol}}^{s_{0}^{-1}} d_{M_{i}}\right) \rightarrow\left(Y, y, s_{0}^{-1}\right.$ $\left.d_{Y}, \hat{v}\right)$. The following claim follows from the proof of the splitting theorem on limit spaces (see for instance [2, Lemmas 9.8, 9.10 and 9.13] or [3]).

Claim 3.3. For every sufficiently large $i$, there exist collections of harmonic functions $\left\{\hat{\mathbf{b}}_{j}^{i}\right\}_{1 \leq j \leq k}$ on $\hat{B}_{100^{2}}\left(w_{i}\right)$, and of points $\left\{x_{j}^{i}\right\}_{2 \leq j \leq k}$ in $\hat{B}_{\sqrt{\delta}^{-1}}\left(w_{i}\right)$ such that $\left|\hat{\mathbf{b}}_{j}^{i}-\hat{r}_{x_{j}^{i}}\right|_{L^{\infty}\left(\hat{B}_{100^{2}}\left(w_{i}\right)\right)} \leq \Psi(\delta ; n)$,

$$
\frac{1}{\hat{\operatorname{vol}} \hat{B}_{100^{2}}\left(w_{i}\right)} \int_{\hat{B}_{100^{2}}\left(w_{i}\right)}\left(\left|\hat{d} \hat{\mathbf{b}}_{j}^{i}-\hat{d} \hat{r}_{x_{j}^{i}}\right|_{s_{0}}^{2}+\left|\operatorname{Hess}_{\hat{\mathbf{b}}_{j}^{i}}\right|_{s_{0}}^{2}\right) d \hat{\operatorname{vol}} \leq \Psi(\delta ; n),
$$

and

$$
\frac{1}{\operatorname{vol} \hat{B}_{100^{2}}\left(w_{i}\right)} \int_{\hat{B}_{100^{2}}\left(w_{i}\right)}\left|\left\langle\hat{d} \hat{\mathbf{b}}_{j}^{i}, \hat{\mathbf{b}}_{l}^{i}\right\rangle_{s_{0}}\right| d \hat{\operatorname{vol}}=\delta_{j l} \pm \Psi(\delta ; n)
$$

for every $1 \leq j \leq l \leq k$, where $x=x_{1}^{i}$ for every $i$.

Define a non-negative valued Borel function $F_{i}$ on $\hat{B}_{100^{2}}\left(w_{i}\right)$ by

$$
F_{i}=\sum_{l=1}^{k} \operatorname{Lip}\left(\hat{\mathbf{b}}_{l}^{i}-\hat{r}_{x_{l}^{i}}\right)^{2}+\sum_{l \neq j}\left|\left\langle\hat{d} \hat{\mathbf{b}}_{l}^{i}, \hat{d} \hat{\mathbf{b}}_{j}^{i}\right\rangle_{s_{0}}\right|+\sum_{l=1}^{k}\left|\operatorname{Hess}_{\hat{\mathbf{b}}_{l}^{i}}\right|_{s_{0}}^{2}
$$


By Lemma 3.1, for every sufficiently large $i$, there exists a compact subset $K_{i}$ of $\hat{\bar{B}}_{100}\left(w_{i}\right)$ such that $\hat{\operatorname{vol}} K_{i} / \hat{\operatorname{vol}} \hat{B}_{100}\left(w_{i}\right) \geq 1-\Psi(\delta ; n)$ and

$$
\frac{1}{\operatorname{vol} \hat{B}_{t}(\alpha)} \int_{\hat{B}_{t}(\alpha)} F_{i} d \hat{\text { vol }} \leq \Psi(\delta ; n)
$$

for every $\alpha \in K_{i}$ and every $0<t<100$.

Claim 3.4. For every sufficiently large $i$, every $\alpha \in K_{i} \cap \hat{B}_{50}\left(w_{i}\right)$, every $1 \leq j \leq k$, and every $0<t<50$, there exists a constant $C_{j}^{i}$ such that $\hat{\mathbf{b}}_{j}^{i}=$ $\hat{r}_{x_{j}^{i}}+C_{j}^{i} \pm \Psi(\delta ; n) t$ on $\hat{B}_{t}(\alpha)$.

The proof is as follows. By the Poincaré inequality, we have

$$
\begin{aligned}
& \frac{1}{\hat{\operatorname{vol}} \hat{B}_{t}(\alpha)} \int_{\hat{B}_{t}(\alpha)}\left|\left(\hat{\mathbf{b}}_{j}^{i}-\hat{r}_{x_{j}^{i}}\right)-\frac{1}{\hat{\operatorname{vol}} \hat{B}_{t}(\alpha)} \int_{\hat{B}_{t}(\alpha)}\left(\hat{\mathbf{b}}_{j}^{i}-\hat{r}_{x_{j}^{i}}\right) d \hat{\operatorname{vol}}\right| d \hat{\operatorname{vol}} \\
& \quad \leq t C(n) \sqrt{\frac{1}{\hat{\operatorname{vol}} \hat{B}_{t}(\alpha)} \int_{\hat{B}_{t}(\alpha)}\left(\operatorname{Lip}\left(\hat{\mathbf{b}}_{1}^{i}-\hat{r}_{x_{i}}\right)\right)^{2} d \hat{\operatorname{vol}}} \\
& \quad \leq t \Psi(\delta ; n) .
\end{aligned}
$$

For $C>0$, let $A_{j}(C)$ be the set of points $\beta \in \hat{B}_{t}(\alpha)$ satisfying that

$$
\left|\left(\hat{\mathbf{b}}_{j}^{i}(\beta)-\hat{r}_{x_{j}^{i}}(\beta)\right)-\frac{1}{\hat{\operatorname{vol}} \hat{B}_{t}(\alpha)} \int_{\hat{B}_{t}(\alpha)}\left(\hat{\mathbf{b}}_{j}^{i}-\hat{r}_{x_{j}^{i}}\right) d \hat{\operatorname{vol}}\right| \geq C .
$$

Then, we have

$$
\begin{aligned}
\Psi(\delta ; n) t & \geq \frac{1}{\hat{\operatorname{vol}} \hat{B}_{t}(\alpha)} \int_{\hat{B}_{t}(\alpha)}\left|\left(\hat{\mathbf{b}}_{j}^{i}-\hat{r}_{x_{j}^{i}}\right)-\frac{1}{\hat{\operatorname{vol}} \hat{B}_{t}(\alpha)} \int_{\hat{B}_{t}(\alpha)}\left(\hat{\mathbf{b}}_{j}^{i}-\hat{r}_{x_{j}^{i}}\right) d \hat{\operatorname{vol}}\right| d \hat{\operatorname{vol}} \\
& \geq C \frac{\hat{\operatorname{vol} A_{j}(C)}}{\hat{\operatorname{vol}} \hat{B}_{t}(\alpha)} .
\end{aligned}
$$

Put $C=\sqrt{\Psi(\delta ; n)} t$ for $\Psi(\delta ; n)$ as above. Then we have vol $A_{j}(C) / \hat{\operatorname{vol}} \hat{B}_{t}(\alpha)$ $\leq \sqrt{\Psi(\delta ; n)}$.

Assume that there exist $\beta \in \hat{B}_{t}(\alpha)$ and $\epsilon>0$ such that $\hat{B}_{\epsilon t}(\beta) \subset A_{j}(C)$. Then, by the Bishop-Gromov volume comparison theorem, we have $C(n) \epsilon^{n} \leq$ $\hat{\operatorname{vol}} B_{\epsilon t}(\beta) / \hat{\operatorname{vol}} \hat{B}_{t}(\alpha) \leq \hat{\operatorname{vol}} A_{j}(C) / \hat{\operatorname{vol}} \hat{B}_{t}(\alpha) \leq \sqrt{\Psi(\delta ; n)}$. Therefore, by letting $\epsilon=\left(2 C(n)^{-1} \sqrt{\Psi(\delta ; n)}\right)^{1 / n}$, we have a contradiction. 
Put $\epsilon=\left(2 C(n)^{-1} \sqrt{\Psi(\delta ; n)}\right)^{1 / n}$. Let $\beta \in \hat{B}_{t}(\alpha)$ and $\hat{\beta} \in \hat{B}_{(1-\epsilon) t}(\alpha)$ with $\hat{r}_{\beta}(\hat{\beta})<\epsilon t$. Then, there exists $\gamma \in \hat{B}_{\epsilon t}(\hat{\beta}) \backslash A_{j}(C)$. Especially, we have $\gamma \in$ $\hat{B}_{t}(\alpha)$. By the definition of $A_{j}(C)$, we have

$$
\hat{\mathbf{b}}_{j}^{i}(\gamma)=\hat{r}_{x_{j}^{i}}(\gamma)+\frac{1}{\hat{\operatorname{vol}} \hat{B}_{100}(\alpha)} \int_{\hat{B}_{100}(\alpha)}\left(\hat{\mathbf{b}}_{j}^{i}-\hat{r}_{x_{j}^{i}}\right) d \hat{\operatorname{vol}} \pm \sqrt{\Psi(\delta ; n)} t .
$$

By Cheng-Yau's gradient estimate (see [7] or [36]), we have $\left|\hat{\nabla} \hat{\mathbf{b}}_{j}^{i}\right|_{s_{0}} \leq C(n)$. Thus, we have

$$
\hat{\mathbf{b}}_{j}^{i}(\beta)=\hat{r}_{x_{j}^{i}}(\beta)+\frac{1}{\hat{\operatorname{vol} \hat{B}_{100}(\alpha)}} \int_{\hat{B}_{100}(\alpha)}\left(\hat{\mathbf{b}}_{j}^{i}-\hat{r}_{x_{j}^{i}}\right) d \hat{\operatorname{vol}} \pm \Psi(\epsilon ; n) t .
$$

Therefore, we have Claim 3.4.

By an argument similar to the proof of [6, Theorem 3.3], we have the following:

Claim 3.5. For every sufficiently large $i$, every $\alpha \in K_{i} \cap \hat{B}_{50}\left(w_{i}\right)$ and every $0<t \leq 10^{-5}$, there exist a compact subset $Z_{t}$ of $M_{i}$, a point $z_{t}$ in $Z_{t}$ and $a$ map $\phi$ from $\left(\hat{\bar{B}}_{t}(\alpha), \alpha\right)$ to $\left(\hat{\bar{B}}_{t}\left(z_{t}\right), z_{t}\right)$ such that the map $\Phi=\left(\hat{\mathbf{b}}_{1}^{i}, \ldots, \hat{\mathbf{b}}_{k}^{i}, \phi\right)$ from $\hat{\bar{B}}_{t}(\alpha)$ to $\hat{\bar{B}}_{t+\Psi(\delta ; n) t}(\Phi(\alpha)) \subset\left(\mathbf{R}^{k} \times Z_{t}, \sqrt{d_{\mathbf{R}^{k}}^{2}+\left(s_{0}-1 d_{M_{i}}\right)^{2}}\right)$, is a $\Psi$ $(\delta ; n)$ t-Gromov-Hausdorff approximation.

Put $\hat{K}_{i}=K_{i} \cap \hat{\bar{B}}_{40}\left(w_{i}\right)$. Then, we have vol $K_{i} / \hat{\operatorname{vol}} \hat{B}_{40}\left(w_{i}\right) \geq 1-\Psi(\delta ; n)$. By Gromov's compactness theorem, without loss of generality, we can assume that there exists a compact subset $K_{\infty}$ of $\hat{\bar{B}}_{40}(w)$ and a collection $\left\{x_{j}^{\infty}\right\}_{2 \leq j \leq k}$ of points in $Y$ such that $x_{j}^{i} \rightarrow x_{j}^{\infty}$ and $K_{i} \rightarrow K_{\infty}$. By Proposition 2.3, we have $\hat{v}\left(K_{\infty}\right) / \hat{v}\left(\hat{B}_{40}(w)\right) \geq 1-\Psi(\delta ; n)$. On the other hand, by Claims 3.4 and 3.5, for every $\alpha \in K_{\infty}$ and every $0<t \leq 10^{-5}$, there exists a compact metric space $Z_{\infty}$, a point $z_{\infty}$ in $Z_{\infty}$, and a map $\phi$ from $\left(\hat{\bar{B}}_{t}(\alpha), \alpha\right)$ to $\left(\bar{B}_{t}\left(z_{\infty}\right), z_{\infty}\right)$ such that the map $\hat{\phi}=\left(\hat{r}_{x}, \hat{r}_{x_{2}^{\infty}}, \ldots, \hat{r}_{x_{k}^{\infty}}, \phi\right)$ from $\hat{\bar{B}}_{t}(\alpha)$ to $\hat{\bar{B}}_{t+\Psi(\delta ; n) t}(\hat{\phi}(\alpha))$, is a $\Psi(\delta ; n) t$-Gromov-Hausdorff approximation. Put $\hat{K}_{\infty}=K_{\infty} \cap\left(\mathcal{R}_{k}\right)_{\delta, r} \cap$ $\bar{B}_{10^{-10} s_{0}}(w)$. Then, we have $v\left(\hat{K}_{\infty}\right) / v\left(\bar{B}_{10^{-10} s_{0}}(w)\right) \geq 1-\Psi(\delta ; n)$. On the other hand, for every $\alpha \in \hat{K}_{\infty}$ and every $0<t \leq 10^{-5}$, let $\phi, Z_{\infty}, z_{\infty}$ as above. Then, since $\alpha \in\left(\mathcal{R}_{k}\right)_{\delta, r}$, we have $\operatorname{diam} Z_{\infty} \leq \Psi(\delta ; n) t$. Especially, the map $f=\left(\hat{r}_{x}, \hat{r}_{x_{2}^{\infty}}, \ldots, \hat{r}_{x_{k}^{\infty}}\right)$ from $\hat{\bar{B}}_{t}(\alpha)$ to $\bar{B}_{t+\Psi(\delta ; n) t}(f(\alpha))$, is a $\Psi(\delta ; n) t$ Gromov-Hausdorff approximation. Especially, for every $\alpha, \beta \in \hat{K}_{\infty}$ with $\alpha \neq$ 
$\beta$, by letting $t=\hat{r}_{\alpha}(\beta)\left(\leq 10^{-5}\right)$, we have

$$
\begin{aligned}
& \sqrt{\left(\overline{x, \alpha} s^{-1} d_{Y}-\overline{x, \beta}^{s_{0}^{-1} d_{Y}}\right)^{2}+\sum_{l=2}^{k}\left(\overline{x_{l}^{\infty}, \alpha} s^{-1} d_{Y}-\bar{x}_{l}^{\infty}, \beta^{s_{0}^{-1}} d_{Y}\right)^{2}} \\
& \quad=\overline{\alpha, \beta}^{s_{0}^{-1} d_{Y}} \pm \Psi(\delta ; n) t \\
& =(1 \pm \Psi(\delta ; n)) \overline{\alpha, \beta}^{s_{0}^{-1} d_{Y}} .
\end{aligned}
$$

Therefore, we have the assertion.

Lemma 3.4. Let $(Y, y, v)$ be a Ricci limit space and $x$ a point in $Y$. Then, there exist collections of compact subsets $\left\{C_{k, i}^{x}\right\}_{1 \leq k \leq n, i \in \mathbf{N}}$ of $Y$, and of points $\left\{x_{k, i}^{l}\right\}_{2 \leq l \leq k \leq n, i \in \mathbf{N}}$ in $Y$ such that the following properties hold:

1. $\bigcup_{i \in \mathbf{N}} C_{k, i}^{x} \subset \mathcal{R}_{k}$ and $v\left(\mathcal{R}_{k} \backslash \bigcup_{i \in \mathbf{N}} C_{k, i}^{x}\right)=0$ for every $k$.

2. For every $z \in \bigcup_{i \in \mathbf{N}} C_{k, i}^{x}$ and every $0<\delta<1$, there exists $C_{k, i}^{x}$ such that $z \in C_{k, i}^{x}$ and that the map $\Phi_{k, i}^{x}=\left(r_{x}, r_{x_{k, i}^{2}}, \ldots, r_{x_{k, i}^{k}}\right)$ from $C_{k, i}^{x}$ to $\mathbf{R}^{k}$, is $(1 \pm \delta)$-bi-Lipschitz to the image $\Phi_{k, i}^{x}\left(C_{k, i}^{x}\right)$.

Proof. Put

$$
A_{k}=\bigcap_{m_{1} \in \mathbf{N}}\left(\bigcup_{m_{2} \in \mathbf{N}}\left(\left(\mathcal{R}_{k}\right)_{1 / m_{1}, 1 / m_{2}}^{x} \cap \operatorname{Leb}\left(\left(\mathcal{R}_{k}\right)_{1 / m_{1}, 1 / m_{2}}\right) \backslash\left(C_{x} \cup\{x\}\right)\right)\right) .
$$

Claim 3.6. We have $A_{k} \subset \mathcal{R}_{k}$ and $v\left(\mathcal{R}_{k} \backslash A_{k}\right)=0$.

The proof is as follows. Put

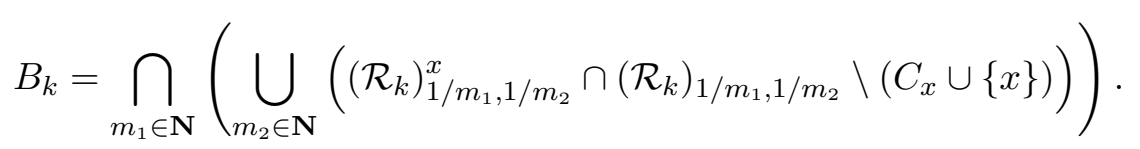

Then we have $A_{k} \subset B_{k}$ and $v\left(B_{k} \backslash A_{k}\right)=0$. On the other hand, by Lemma 3.2, we have $B_{k}=\mathcal{R}_{k} \backslash\left(C_{x} \cup\{x\}\right)$. Since $v\left(C_{x}\right)=0$, we have Claim 3.6.

For every $z \in A_{k}$ and every $N \in \mathbf{N}$, there exists $m_{2}=m_{2}(z, N)$ such that $z \in\left(\mathcal{R}_{k}\right)_{1 / N, 1 / m_{2}}^{x} \cap \operatorname{Leb}\left(\left(\mathcal{R}_{k}\right)_{1 / N, 1 / m_{2}}\right) \backslash\left(C_{x} \cup\{x\}\right)$. By Lemma 3.3, there exists $\eta(z, N)>0$ such that for every $0<s \leq \eta(z, N)$, there exists a compact subset $L(z, s, N)$ of $\bar{B}_{s}(z) \cap\left(\mathcal{R}_{k}\right)_{1 / N, 1 / m_{2}}$ and a collection of points $\left\{x_{j}(z, s, N)\right\}_{1 \leq j \leq k}$ in $Y$ such that $v(L(z, s, N)) / v\left(\bar{B}_{s}(z)\right) \geq 1-\Psi\left(N^{-1} ; n\right)$ and that the map $\Phi_{z, s, N}=\left(r_{x}, r_{x_{2}(z, s, N)} \ldots, r_{x_{k}(z, s, N)}\right)$ from $L(z, s, N)$ to 
$\mathbf{R}^{k}$, is $\left(1 \pm \Psi\left(N^{-1} ; n\right)\right)$-bi-Lipschitz to the image. Fix $R>1$ and $N \in \mathbf{N}$. By Lemma 2.2, there exists a pairwise disjoint collection $\left\{\bar{B}_{s_{i}^{N . R}}\left(z_{i}^{N, R}\right)\right\}_{i \in \mathbf{N}}$ such that $z_{i}^{N, R} \in A_{k} \cap \bar{B}_{R}(y), 0<s_{i}^{N, R} \leq \eta\left(z_{i}^{N, R}, N\right) / 100$ and $A_{k} \cap \bar{B}_{R}(y) \backslash$ $\bigcup_{i=1}^{m} \bar{B}_{s_{i}^{N, R}}\left(z_{i}^{N, R}\right) \subset \bigcup_{i=m+1}^{\infty} \bar{B}_{5 s_{i}^{N, R}}\left(z_{i}^{N, \bar{R}}\right)$ for every $m$. Put $\hat{L}(i, N, R)=$ $L\left(z_{i}^{N, R}, 5 s_{i}^{N, R}, N\right) \cap A_{k} \cap \bar{B}_{R}(y) \subset A_{k} \cap \bar{B}_{R}(y)$.

Claim 3.7. $v\left(A_{k} \cap \bar{B}_{R}(y) \backslash \bigcup_{N \geq N_{0}, i \in \mathbf{N}} \hat{L}(i, N, R)\right)=0$ for every $N_{0} \in \mathbf{N}$.

Because we have

$$
\begin{aligned}
& v\left(A_{k} \cap \bar{B}_{R}(y) \backslash \bigcup_{i \in \mathbf{N}} \hat{L}(i, N, R)\right) \\
& \leq v\left(\bigcup_{i \in \mathbf{N}}\left(\bar{B}_{5 s_{i}^{N, R}}\left(z_{i}^{N, R}\right) \cap A_{k} \cap \bar{B}_{R}(y)\right) \backslash\right. \\
& \left.\quad \bigcup_{i \in \mathbf{N}}\left(L\left(z_{i}^{N, R}, 5 s_{i}^{N, R}, N\right) \cap A_{k} \cap \bar{B}_{R}(y)\right)\right) \\
& \leq \sum_{i \in \mathbf{N}} v\left(\bar{B}_{5 s_{i}^{N, R}}\left(z_{i}^{N, R}\right) \backslash L\left(z_{i}^{N, R}, 5 s_{i}^{N, R}, N\right)\right) \\
& \leq \Psi\left(N^{-1} ; n\right) \sum_{i \in \mathbf{N}} v\left(\bar{B}_{5 s_{i}^{N, R}}\left(z_{i}^{N, R}\right)\right) \leq \Psi\left(N^{-1} ; n\right) \sum_{i \in \mathbf{N}} v\left(B_{s_{i}^{N, R}}\left(z_{i}^{N, R}\right)\right) \\
& \quad \leq \Psi\left(N^{-1} ; n\right) v\left(B_{2 R}(y)\right)
\end{aligned}
$$

for every $N \geq N_{0}$. Therefore, by letting $N \rightarrow \infty$, we have Claim 3.7.

By Claim 3.7, we have $v\left(A_{k} \cap \bar{B}_{R}(y) \backslash \bigcap_{N_{0}}\left(\bigcup_{N \geq N_{0}, i \in \mathbf{N}} \hat{L}(i, N, R)\right)\right)=$ 0 . Put $E(i, N, R)=\hat{L}(i, N, R) \cap \bigcap_{N_{0} \in \mathbf{N}}\left(\bigcup_{N \geq N_{0}, j \in \mathbf{N}} \hat{L}(j, N, R)\right)$. Then, we have $v\left(A_{k} \cap \bar{B}_{R}(y) \backslash \bigcup_{i, N \in \mathbf{N}} E(i, N, R)\right)=0$. Fix $z \in \bigcup_{i, N \in \mathbf{N}} E(i, N, R)$ and $0<\delta<1$. Then there exist $i, N$ such that $z \in E(i, N, R)$. Let $N_{0} \in \mathbf{N}$ with $N_{0}^{-1} \ll \delta$. Then there exist $\hat{N} \geq N_{0}$ and $\hat{i} \in \mathbf{N}$ such that $z \in \hat{L}(\hat{i}, \hat{N}, R)$. By the definition, the map $\phi=\left(r_{x}, r_{x_{2}\left(z_{\hat{i}}^{\hat{N}, R}, s_{\hat{i}}^{\hat{N}, R}\right)}, \ldots, r_{x_{k}\left(z_{\hat{i}}^{\hat{N}, R}, s_{\hat{\imath}}^{\hat{N}, R}\right)}\right)$ from $L\left(z_{\hat{i}}^{\hat{N}, R}, s_{\hat{i}}^{\hat{N}, R}, \hat{N}\right)$ to $\mathbf{R}^{k}$, is $\Psi\left(N^{-1}, n\right)$-bi-Lipschitz to the image. Especially, the map is $(1 \pm \delta)$-bi-Lipschitz to the image. We remark that $\hat{L}(\hat{i}, \hat{N}, R) \subset$ $L\left(z_{\hat{i}}^{\hat{N}, R}, s_{\hat{i}}^{\hat{N}, R}, \hat{N}\right)$ and $z \in \hat{L}(\hat{i}, \hat{N}, R) \cap \bigcap_{l \in \mathbf{N}}\left(\bigcup_{j \geq l, p \in \mathbf{N}} \hat{L}(p, j, R)\right)=E(\hat{i}, \hat{N}$, $R)$. Therefore, by letting $x_{j}(i, N, R)=x_{j}\left(z_{i}^{N, R}, s_{i}^{N, R}, R\right)$ for every $2 \leq j \leq k$, we have the following claim: 
Claim 3.8. For every $z \in \bigcup_{i, N \in \mathbf{N}} E(i, N, R)$ and every $0<\delta<1$, there exists $E(i, N, R)$ such that $z \in E(i, N, R)$ and that the map $\phi=\left(r_{x}\right.$, $\left.r_{x_{2}(i, N, R)}, \ldots, r_{x_{k}(i, N, R)}\right)$ from $E(i, N, R)$ to $\mathbf{R}^{k}$, is $(1 \pm \delta)$-bi-Lipschitz to the image.

By Claim 3.8, it is easy to check the assertion.

Lemma 3.5. With the same notaion as in Lemma 3.4, for every $k$, let $\left\{\mathcal{F}_{k, i, j}^{x}\right\}_{j \in \mathbf{N}}$ be a collection of Borel subsets of $C_{k, i}^{x}$ with $v\left(C_{k, i}^{x} \backslash \bigcup_{j \in \mathbf{N}} \mathcal{F}_{k, i, j}^{x}\right)=0$. Then, there exists a collection of Borel subsets $\left\{\mathcal{E}_{k, i, j}^{x}\right\}_{k, i, j}$ of $Y$ such that $\mathcal{E}_{k, i, j}^{x} \subset \mathcal{F}_{k, i, j}^{x}, v\left(\mathcal{F}_{k, i, j}^{x} \backslash \mathcal{E}_{k, i, j}^{x}\right)=0$ and that for every $k$, every $z \in \bigcup_{i, j \in \mathbf{N}} \mathcal{E}_{k, i, j}^{x}$ and every $0<\delta<1$, there exists $\mathcal{E}_{k, i, j}^{x}$ such that $z \in \mathcal{E}_{k, i, j}^{x}$ and that the map $\Phi_{k, i, j}^{x}=\left(r_{x}, r_{x_{k, i}^{2}}, \ldots, r_{x_{k, i}^{k}}\right)$ from $\mathcal{E}_{k, i, j}^{x}$ to $\mathbf{R}^{k}$, is $(1 \pm \delta)$-bi-Lipschitz to the image.

Proof. Fix $1 \leq k \leq n$. For every $M \in \mathbf{N}$, put $\mathcal{B}_{M}=\{i \in \mathbf{N}$; The map $\phi=$ $\left(r_{x}, r_{x_{k, i}^{2}}, \ldots, r_{x_{k, i}^{k}}\right)$ from $C_{k, i}^{x}$ to $\mathbf{R}^{k}$, is $\left(1 \pm M^{-1}\right)$-bi-Lipschitz to the image $\}$ and $\mathcal{E}_{k, i, j}^{x}=\mathcal{F}_{k, i, j}^{x} \cap \bigcap_{M \in \mathbf{N}}\left(\bigcup_{i \in \mathcal{B}_{M}, j \in \mathbf{N}} \mathcal{F}_{k, i, j}^{x}\right)$.

Claim 3.9. $v\left(\mathcal{F}_{k, i, j}^{x} \backslash \mathcal{E}_{k, i, j}^{x}\right)=0$.

The proof is as follows. By Lemma 3.4, we have $\bigcup_{i \in \mathbf{N}} C_{k, i}^{x} \subset \bigcap_{M \in \mathbf{N}}$ $\left(\bigcup_{i \in \mathcal{B}_{M}} C_{k, i}^{x}\right)$. On the other hand, it is easy to check that $\bigcap_{M \in \mathbf{N}}$ $\left(\bigcup_{i \in \mathcal{B}_{M}} C_{k, i}^{x}\right) \subset \bigcup_{i \in \mathbf{N}} C_{k, i}^{x}$. Therefore, we have $\bigcap_{M \in \mathbf{N}}\left(\bigcup_{i \in \mathcal{B}_{M}} C_{k, i}^{x}\right)=$ $\bigcup_{i \in \mathbf{N}} C_{k, i}^{x}$. Thus, $v\left(\mathcal{F}_{k, i, j}^{x} \backslash \mathcal{E}_{k, i, j}^{x}\right)=v\left(\mathcal{F}_{k, i, j}^{x} \cap \bigcup_{l \in \mathbf{N}} C_{k, l}^{x} \backslash \mathcal{E}_{k, i, j}^{x}\right)=v\left(\mathcal{F}_{k, i, j}^{x} \cap\right.$ $\left.\bigcap_{M \in \mathbf{N}}\left(\bigcup_{l \in \mathcal{B}_{M}} C_{k, l}^{x}\right) \backslash \mathcal{E}_{k, i, j}^{x}\right)=v\left(\mathcal{F}_{k, i, j}^{x} \cap \bigcap_{M \in \mathbf{N}}\left(\bigcup_{l \in \mathcal{B}_{M}, j \in \mathbf{N}} \mathcal{F}_{k, l, j}^{x}\right) \backslash \mathcal{E}_{k, i, j}^{x}\right)$ $=0$. Therefore, we have Claim 3.9.

Claim 3.10. For every $z \in \bigcup_{i, j \in \mathbf{N}} \mathcal{E}_{k, i, j}^{x}$ and every $0<\delta<1$, there exists $\mathcal{E}_{k, i, j}^{x}$ such that $z \in \mathcal{E}_{k, i, j}^{x}$ and that the map $\phi=\left(r_{x}, r_{x_{k, i}^{2}}, \ldots, r_{x_{k, i}^{k}}\right)$ from $\mathcal{E}_{k, i, j}^{x}$ to $\mathbf{R}^{k}$, is $(1 \pm \delta)$-bi-Lipschitz to the image.

The proof is as follows. Let $M, i, j$ be positive integers with $M^{-1} \ll \delta$, $z \in \mathcal{E}_{k, i, j}^{x}$. There exist $N_{0} \in \mathcal{B}_{M}$ and $N_{1} \in \mathbf{N}$ such that $z \in \mathcal{F}_{k, N_{0}, N_{1}}^{x}$. Therefore, we have $z \in \mathcal{F}_{k, N_{0}, N_{1}}^{x} \cap \bigcap_{\hat{M} \in \mathbf{N}}\left(\bigcup_{\hat{i} \in \mathcal{B}_{\hat{M}}, \hat{j} \in \mathbf{N}} \mathcal{F}_{k, \hat{i}, \hat{j}}^{x}\right)=\mathcal{E}_{k, N_{0}, N_{1}}^{x}$ and that the map $\phi=\left(r_{x}, r_{x_{k, j}^{2}}, \ldots, r_{x_{k, j}^{k}}\right)$ from $\mathcal{E}_{k, N_{0}, N_{1}}^{x}$ to $\mathbf{R}^{k}$, is $\left(1 \pm M^{-1}\right)$-biLipschitz to the image. Thus, we have Claim 3.10.

By Claims 3.9 and 3.10, we have the assertion. 
The following theorem is the main result in this subsection. See (2.2) in [5] or [22, Definition 4.1] for the definition of the measure $v_{-1}$.

Theorem 3.1 Radial rectifiability. Let $(Y, y, v)$ be a Ricci limit space with $Y \neq\{y\}$, and $x$ a point in $Y$. Then, there exist collections of Borel subsets $\left\{C_{k, i}^{x}\right\}_{1 \leq k \leq n, i \in \mathbf{N}}$ of $Y$, of points $\left\{x_{k, i}^{l}\right\}_{2 \leq l \leq k \leq n, i \in \mathbf{N}}$ in $Y$, a positive number $0<\alpha(n)<1$ and a Borel subset $A$ of $[0, \operatorname{diam} Y)$ such that the following properties hold:

1. $\bigcup_{i \in \mathbf{N}} C_{k, i}^{x} \subset \mathcal{R}_{k, \alpha(n)} \backslash C_{x}$ and $v\left(\mathcal{R}_{k} \backslash \bigcup_{i \in \mathbf{N}} C_{k, i}^{x}\right)=0$ for every $k$.

2. $\lim _{r \rightarrow 0} v\left(B_{r}(z) \cap C_{k, i}^{x}\right) / v\left(B_{r}(z)\right)=1$ for every $C_{k, i}^{x}$ and every $z \in C_{k, i}^{x}$.

3. For every $C_{k, i}^{x}$, there exists $A_{k, i}^{x}>1$ such that $\left(A_{k, i}^{x}\right)^{-1} \leq v\left(B_{r}(z)\right) /$ $r^{k} \leq A_{k, i}^{x}$ for every $z \in C_{k, i}^{x}$ and every $0<r<1$.

4. The limit measure $v$ and the $k$-dimensional Hausdorff measure $H^{k}$ are mutually absolutely continuous on $C_{k, i}^{x}$.

5. For every $z \in \bigcup_{i \in \mathbf{N}} C_{k, i}^{x}$ and every $0<\delta<1$, there exists $C_{k, i}^{x}$ such that $z \in C_{k, i}^{x}$ and that the map $\Phi_{k, i}^{x}=\left(r_{x}, r_{x_{k, i}^{2}}, \ldots, r_{x_{k, i}^{k}}\right)$ from $C_{k, i}^{x}$ to $\mathbf{R}^{k}$, is $(1 \pm \delta)$-bi-Lipschitz to the image.

6. $H^{1}([0, \operatorname{diam} Y) \backslash A)=0$.

7. For every $R \in A$, the collection $\left\{\partial B_{R}(x) \cap C_{k, i}^{x}\right\}_{k, i} \subset \partial B_{R}(x) \backslash C_{x}$ satisfies the following properties:

(a) $v_{-1}\left(\left(\partial B_{R}(x) \backslash C_{x}\right) \backslash \bigcup_{1 \leq k \leq n, i \in \mathbf{N}} C_{k, i}^{x}\right)=0$.

(b) For every $\partial B_{R}(x) \cap C_{k, i}^{x}$, there exist $B_{k, i}^{x}>1$ and $\tau_{k, i}^{x}>0$ such that $\left(B_{k, i}^{x}\right)^{-1} \leq v_{-1}\left(\partial B_{R}(x) \cap B_{r}(z) \backslash C_{x}\right) / r^{k-1} \leq v_{-1}\left(\partial B_{R}(x) \cap \bar{B}_{r}(z)\right) /$ $r^{k-1} \leq B_{k, i}^{x}$ for every $z \in \partial B_{R}(x) \cap C_{k, i}^{x}$ and every $0<r<\tau_{k, i}^{x}$.

(c) For every $z \in \bigcup_{i \in \mathbf{N}}\left(\partial B_{R}(x) \cap C_{k, i}^{x}\right)$ and every $0<\delta<1$, there exists $\partial B_{R}(x) \cap C_{k, i}^{x}$ such that $z \in \partial B_{R}(x) \cap C_{k, i}^{x}$ and that the map $\hat{\Phi}_{k, i}^{x}=$ $\left(r_{x_{k, i}^{2}}, \ldots, r_{x_{k, i}^{k}}\right)$ from $\partial B_{R}(x) \cap C_{k, i}^{x}$ to $\mathbf{R}^{k-1}$, is $(1 \pm \delta)$-bi-Lipschitz to the image.

Especially, $\partial B_{R}(x) \backslash C_{x}$ is $v_{-1}$-rectifiable.

Proof. First, we shall prove the following claim:

Claim 3.11. We have $v_{-1}\left(\partial B_{\overline{x, z}}(x) \cap \bar{B}_{\epsilon}(z)\right) \leq C(n) v\left(B_{\epsilon}(z)\right) / \epsilon$ for every $R>0$, every $z \in \bar{B}_{R}(x) \backslash\{x\}$ and every $\epsilon>0$ with $\epsilon<\min \{\overline{z, x} / 100,1\}$. 
The proof is as follows. By [23, Corollary 5.7], we have

$$
\frac{v_{-1}\left(\partial B_{\overline{x, z}}(x) \cap \bar{B}_{\epsilon}(z)\right)}{\operatorname{vol} \partial B_{\overline{x, z}}(\underline{p})} \leq C(n) \frac{v\left(C_{x}\left(\partial B_{\overline{x, z}}(x) \cap \bar{B}_{\epsilon}(z)\right) \cap A_{\overline{x, z}-2 \epsilon, \overline{x, z}}(x)\right)}{\operatorname{vol} A_{\overline{x, z}-2 \epsilon, \overline{x, z}}(\underline{p})} .
$$

Here $C_{x}(A)=\{z \in Y$; There exists $a \in A$ such that $\overline{x, z}+\overline{z, a}=\overline{z, a}\}$ for every subset $A$ of $Y, \underline{p}$ is a point in the $n$-dimensional hyperbolic space form. On the other hand, by triangle inequality, we have $C_{x}\left(\partial B_{\overline{x, z}}(x) \cap\right.$ $\left.\bar{B}_{\epsilon}(z)\right) \cap A_{\overline{x, z}-2 \epsilon, \overline{x, z}}(x) \subset \bar{B}_{100 \epsilon}(z)$. Thus, we have

$v_{-1}\left(\partial B_{\overline{x, z}}(x) \cap \bar{B}_{\epsilon}(z)\right) \leq \frac{\operatorname{vol} \partial B_{\overline{x, z}}(\underline{p})}{\operatorname{vol} A_{\overline{x, z}-2 \epsilon, \overline{x, z}}(\underline{p})} v\left(B_{100 \epsilon}(z)\right) C(n) \leq C(n, R) \frac{1}{\epsilon} v\left(B_{\epsilon}(z)\right)$.

Therefore, we have Claim 3.11.

Let $\left\{C_{k, i}^{x}\right\}_{k, i}$ be a collection of Borel subsets of $Y$ and $\left\{x_{k, i}^{l}\right\}_{k, i, l}$ a collection of points in $Y$ as in Lemma 3.4. By Lemma 3.5, without loss of generality, we can assume that for every $C_{k, i}^{x}$, there exists $\tau>0$ such that $C_{k, i}^{x} \subset \mathcal{D}_{x}^{\tau} \backslash B_{\tau}(x)$. Moreover, by [6, Theorems 3.23 and 4.6], we can assume that for every $C_{k, i}^{x}$, there exists $A_{k, i}^{x}>1$ such that $\left(A_{k, i}^{x}\right)^{-1} \leq v\left(B_{r}(z)\right) / r^{k} \leq$ $A_{k, i}^{x}$ for every $0<r<1$ and every $z \in C_{k, i}^{x}$, and that $\lim _{r \rightarrow 0} v\left(B_{r}(z) \cap C_{k, i}^{x}\right) /$ $v\left(B_{r}(z)\right)=1$ for every $C_{k, i}^{x}$ and every $z \in C_{k, i}^{x}$.

Claim 3.12. Let $(Y, y, v)$ be a Ricci limit space, $x$ a point in $Y, \tau, R$ positive numbers with $0<\tau<1<R$, and $z$ a point in $\mathcal{D}_{x}^{\tau} \cap B_{R}(x) \backslash B_{\tau}(x)$. Then, we have $v_{-1}\left(\partial B_{\overline{x, z}}(x) \cap B_{\epsilon}(z) \backslash C_{x}\right) \geq C(n, R) v\left(B_{\epsilon}(z)\right) / \epsilon$ for every $0<\epsilon<$ $\tau / 100$.

The proof is as follows. Let $w \in Y$ with $\overline{z, w}=\epsilon / 100, \overline{x, z}+\overline{z, w}=\overline{x, w}$. By [23, Theorem 4.6 ], we have

$$
\frac{v\left(B_{\overline{1} \epsilon}(w)\right)}{\operatorname{vol} A_{\overline{x, z}, \overline{x, z}+\epsilon}(\underline{p})} \leq C(n) \frac{v_{-1}\left(C_{x}\left(B_{\frac{\epsilon}{1000}}(w)\right) \cap \partial B_{\overline{x, z}}(x)\right)}{\operatorname{vol} \partial B_{\overline{x, z}}(\underline{p})} .
$$

By triangle inequality, we have $C_{x}\left(B_{\epsilon / 1000}(w)\right) \cap \partial B_{\overline{x, z}}(x) \subset \partial B_{\overline{x, z}}(x) \cap B_{\epsilon}(z)$. Thus, by the Bishop-Gromov volume comparison theorem for $v$, we have

$$
\begin{aligned}
v_{-1}\left(\partial B_{\overline{x, z}}(x) \cap B_{\epsilon}(z) \backslash C_{x}\right) & \geq C(n) \frac{\operatorname{vol} \partial B_{\overline{x, z}}(\underline{p})}{\operatorname{vol} A_{\overline{x, z}, \overline{x, z}+\epsilon}(\underline{p})} v\left(B_{\epsilon / 1000}(w)\right) \\
& \geq C(n, R) \frac{1}{\epsilon} v\left(B_{\overline{\frac{\epsilon}{1000}}}(w)\right) \\
& \geq C(n, R) \frac{1}{\epsilon} v\left(B_{5 \epsilon}(w)\right) \geq C(n, R) \frac{v\left(B_{\epsilon}(z)\right)}{\epsilon} .
\end{aligned}
$$


Therefore, we have Claim 3.12.

By Claims 3.11 and 3.12, for every $C_{k, i}^{x}$, there exist $B_{k, i}^{x}>1$ and $\tau_{k, i}^{x}>$ 0 such that $\left(B_{k, i}^{x}\right)^{-1} \leq v_{-1}\left(\partial B_{\overline{x, z}}(x) \cap B_{r}(z) \backslash C_{x}\right) / r^{k} \leq B_{k, i}^{x}$ for every $z \in$ $C_{k, i}^{x}$ and every $0<r<\tau_{k, i}^{x}$. Put $\hat{A}=\left\{t \in[0, \operatorname{diam} Y) ; v_{-1}\left(\partial B_{t}(x) \backslash \bigcup C_{k, i}^{x}\right)=\right.$ $0\}$. Since $v\left(Y \backslash \cup C_{k, i}^{x}\right)=0$, it follows from [23, Proposition 5.1 and Theorem 5.2] that $\hat{A}$ is Lebesgue measurable and that $H^{1}([0, \operatorname{diam} Y) \backslash \hat{A})=0$. Since $H^{1}$ is a Radon measure on $\mathbf{R}$, we have the assertion.

\subsection{Calculation of radial derivatives of Lipschitz functions}

The purpose in this subsection is to calculate the radial derivative from a given point $x$, of a given Lipschitz function $f:\left\langle d r_{x}, d f\right\rangle$ explicitly. The main result in this subsection is Theorem 3.3.

Lemma 3.6. Let $(Y, y)$ be a Ricci limit space with $Y \neq\{y\}$, z a point in $Y \backslash C_{y}$, $f$ a Lipschitz function on $Y, \tau$ a positive number and $\gamma_{i}$ an isometric embedding from $[0, \overline{y, z}+\tau]$ to $Y$ satisfying $\gamma_{i}(0)=y, \gamma_{i}(\overline{y, z})=z$ for every $i \in\{1,2\}$. Put $f_{i}=f \circ \gamma_{i}$. Then, we have $\operatorname{lip} f_{1}(\overline{y, z})=\operatorname{lip} f_{2}(\overline{y, z})$ and $\operatorname{Lip} f_{1}(\overline{y, z})=\operatorname{Lip} f_{2}(\overline{y, z})$.

Proof. For every real number $\epsilon$ with $0<|\epsilon| \ll \tau$, by the splitting theorem on limit space, we have $\overline{\gamma_{1}(\overline{x, z}+\epsilon), \gamma_{2}(\overline{x, z}+\epsilon)} \leq \Psi(|\epsilon| ; n)|\epsilon|$. Therefore, we have

$$
\frac{\left|f_{1}(\overline{x, z}+\epsilon)-f a_{1}(\overline{x, z})\right|}{|\epsilon|} \leq \frac{\left|f_{2}(\overline{x, z}+\epsilon)-f_{2}(\overline{x, z})\right|}{|\epsilon|}+\operatorname{Lip} f \Psi(|\epsilon| ; n) .
$$

Thus, we have $\operatorname{Lip} f_{1}(\overline{y, z}) \leq \operatorname{Lip} f_{2}(\overline{y, z})$ and $\operatorname{lip} f_{1}(\overline{y, z}) \leq \operatorname{lip} f_{2}(\overline{y, z})$. Therefore, we have $\operatorname{Lip} f_{1}(\overline{y, z})=\operatorname{Lip} f_{2}(\overline{y, z})$ and $\operatorname{lip} f_{1}(\overline{y, z})=\operatorname{lip} f_{2}(\overline{y, z})$.

Let $(Y, y)$ be a Ricci limit space, $z$ a point in $Y \backslash C_{y}, \tau$ a positive number, $\gamma$ an isometric embedding from $[0, \overline{y, z}+\tau]$ to $Y$ satisfying $\gamma(0)=y$, $\gamma(\overline{y, z})=z$. Put $F=f \circ \gamma, \operatorname{lip} y_{y}^{\operatorname{rad}} f(z)=\operatorname{lip} F(\overline{y, z})$ and $\operatorname{Lip}_{y}^{\operatorname{rad}} f(z)=\operatorname{Lip} F(\overline{y, z})$.

It is not difficult to check the following lemma:

Lemma 3.7. Let $(Z, v)$ be a metric measure space. Assume that the following properties hold:

1. $v\left(B_{r}(z)\right)>0$ for every $z \in Z$ and every $r>0$.

2. There exist $r_{0}>0$ and $\kappa>1$ such that $v\left(B_{2 r}(z)\right) \leq 2^{\kappa} v\left(B_{r}(z)\right)$ for every $z \in Z$ and every $0<r<r_{0}$. 
Then, we have $\operatorname{Lip} f(a)=\operatorname{Lip}\left(\left.f\right|_{A}\right)(a)$ and $\operatorname{lip} f(a)=\operatorname{lip}\left(\left.f\right|_{A}\right)(a)$ for every $a \in \operatorname{Leb}(A)$, every Lipschitz function $f$ on $Z$ and every Borel subset $A$ of $Z$.

The following theorem implies that $\partial B_{R}(x) \perp \nabla r_{x}$ in some sense:

Theorem 3.2. Let $(Y, y, v)$ be a Ricci limit space, $x$ a point in $Y$ and $f a$ Lipschitz function on $Y$. Then, we have the following:

1. $\operatorname{lip} f(z)^{2}=\operatorname{lip} p_{x}^{\mathrm{rad}} f(z)^{2}+\operatorname{lip}\left(\left.f\right|_{\partial B_{\bar{x}, z}(x)}\right)(z)^{2}$ for a.e. $z \in Y$.

2. $\operatorname{Lip} f(z)^{2}=\operatorname{Lip}_{x}^{\mathrm{rad}} f(z)^{2}+\operatorname{Lip}\left(\left.f\right|_{\partial B_{\bar{x}, z}(x)}\right)(z)^{2}$ for a.e. $z \in Y$.

3. $\operatorname{Lip}\left(\left.f\right|_{\partial B_{\overline{x, z}}(x)}\right)(z)=\operatorname{lip}\left(\left.f\right|_{\partial B_{\bar{x}, z}(x) \backslash C_{x}}\right)(z)$ for a.e. $z \in Y \backslash C_{x}$.

Proof. First, we shall remark the following:

Claim 3.13. Let $f$ be a Lipschitz function on $\mathbf{R}^{k}$. Then, we have $\operatorname{Lip} f(z)^{2}=$ $\left(\operatorname{Lip}\left(\left.f\right|_{\mathbf{R} \times\left\{z_{2}, \ldots, z_{k}\right\}}\right)(z)\right)^{2}+\left(\operatorname{Lip}\left(\left.f\right|_{\left\{z_{1}\right\} \times \mathbf{R}^{k-1}}\right)(z)\right)^{2}=\left(\operatorname{lip}\left(\left.f\right|_{\mathbf{R} \times\left\{z_{2}, \ldots, z_{k}\right\}}\right)(z)\right)^{2}+$ $\left(\operatorname{lip}\left(\left.f\right|_{\left\{z_{1}\right\} \times \mathbf{R}^{k-1}}\right)(z)\right)^{2}=\operatorname{lipf}(z)^{2}$ for a.e $z=\left(z_{1}, \ldots, z_{k}\right) \in \mathbf{R}^{k}$.

Because, by Rademacher's theorem about differentiability of Lipschitz functions on $\mathbf{R}^{k}, f$ is totally differentiable at a.e $z \in \mathbf{R}^{k}$. Therefore, we have Claim 3.13.

The next claim is clear:

Claim 3.14. Let $\left\{Z_{i}\right\}_{i=1,2}$ be metric spaces, $\delta$ a positive number with $0<\delta<1$, and $\Phi$ a map from $Z_{1}$ to $Z_{2}$ satisfying that $\Phi\left(Z_{1}\right)=Z_{2}$ and $(1-\delta) \overline{x_{1}, x_{2}} \leq \overline{\Phi\left(x_{1}\right), \Phi\left(x_{2}\right)} \leq(1+\delta) \overline{x_{1}, x_{2}}$ for every $x_{1}, x_{2} \in Z_{1}$. Then, for every Lipschitz function $f$ on $Z_{2}$, we have, $(1-\Psi(\delta)) \operatorname{Lip} f\left(\Phi\left(z_{1}\right)\right) \leq \operatorname{Lip}(f \circ$ $\Phi)\left(z_{1}\right) \leq(1+\Psi(\delta)) \operatorname{Lip} f\left(z_{1}\right),(1-\Psi(\delta)) \operatorname{lip} f\left(\Phi\left(z_{1}\right)\right) \leq \operatorname{lip}(f \circ \Phi)\left(z_{1}\right) \leq(1+$ $\Psi(\delta)) \operatorname{lip} f\left(\Phi\left(z_{1}\right)\right)$ for every $z_{1} \in Z_{1}$.

We will give a proof of the following claim in the Appendix:

Claim 3.15. For every Lebesgue measurable subset $A$ of $\mathbf{R}^{k}$, put $s l_{1}-\operatorname{Leb} A=\left\{a=\left(a_{1}, \ldots, a_{k}\right) \in A ; \lim _{r \rightarrow 0} H^{k-1}\left(\left(\left\{a_{1}\right\} \times \bar{B}_{r}\left(a_{2}, \ldots, a_{k}\right)\right) \cap\right.\right.$ A) $\left./ H^{k-1}\left(\left\{a_{1}\right\} \times \bar{B}_{r}\left(a_{2}, \ldots, a_{k}\right)\right)=1\right\}$. Then the following properties hold:

1. $s l_{1}-\operatorname{Leb} A$ is a Lebesgue measurable set.

2. $H^{k-1}\left(A \cap\left(\{t\} \times \mathbf{R}^{k-1} \backslash s l_{1}-\operatorname{Leb} A\right)\right)=0$ for every $t \in \mathbf{R}$.

3. $H^{k}\left(A \backslash s l_{1}-\operatorname{Leb} A\right)=0$. 
Put $L=\operatorname{Lip} f$. Let $\left\{C_{k, i}^{x}\right\}_{1 \leq k \leq n, i \in \mathbf{N}}$ be a collection of Borel subsets of $Y$, and $\left\{x_{k, i}^{l}\right\}_{2 \leq k \leq n, i \in \mathbf{N}, 2 \leq l \leq k}$ a collection of points in $Y$ as in Theorem 3.1. Fix a sufficiently small $\delta>0$ and $C_{k, i}$ satisfying that the map $\Phi_{k, i}^{x}=$ $\left(r_{x}, r_{x_{k, i}^{2}}, \ldots, r_{x_{k, i}^{k}}\right)$ from $C_{k, i}^{x}$ to $\mathbf{R}^{k}$, is $(1 \pm \delta)$-bi-Lipschitz to the image. Put $f_{k, i}^{x}=f \circ\left(\Phi_{k, i}^{x}\right)^{-1}$ on $\Phi_{k, i}^{x}\left(C_{k, i}^{x}\right)$. Let $F_{k, i}^{x}$ be a Lipschitz function on $\mathbf{R}^{k}$ satisfying that $\left.F_{k, i}^{x}\right|_{\Phi_{k, i}^{x}\left(C_{k, i}^{x}\right)}=f_{k, i}^{x}$ and $\mathbf{L i p} F_{k, i}^{x}=\mathbf{L i p} f_{k, i}^{x}$.

Claim 3.16. With the notation as above, we have the following:

1. $(1-\Psi(\delta ; n)) \operatorname{Lip} F_{k, i}^{x}(w) \leq \operatorname{Lip} f\left(\left(\Phi_{k, i}^{x}\right)^{-1}(w)\right) \leq(1+\Psi(\delta ; n)) \operatorname{Lip}$ $F_{k, i}^{x}(w)$ for a.e $w \in \Phi_{k, i}^{x}\left(C_{k, i}^{x}\right)$.

2. $(1-\Psi(\delta ; n)) \operatorname{lip} F_{k, i}^{x}(w) \leq \operatorname{lipf}\left(\left(\Phi_{k, i}^{x}\right)^{-1}(w)\right) \leq(1+\Psi(\delta ; n)) \operatorname{lip} F_{k, i}^{x}(w)$ for a.e $w \in \Phi_{k, i}^{x}\left(C_{k, i}^{x}\right)$.

3. $\operatorname{Lip}\left(\left.F_{k, i}^{x}\right|_{\mathbf{R} \times\left\{w_{2}, \ldots, w_{k}\right\}}\right)(w)-L \Psi(\delta ; n) \leq \operatorname{Lip}_{x}^{\mathrm{rad}} f\left(\left(\Phi_{k, i}^{x}\right)^{-1}(w)\right) \leq \operatorname{Lip}$ $\left(\left.F_{k, i}^{x}\right|_{\mathbf{R} \times\left\{w_{2}, \ldots, w_{k}\right\}}\right)(w)+L \Psi(\delta ; n)$ for a.e $w=\left(w_{1}, \ldots, w_{k}\right) \in \Phi_{k, i}^{x}\left(C_{k, i}^{x}\right)$.

4. $\operatorname{lip}\left(\left.F_{k, i}^{x}\right|_{\mathbf{R} \times\left\{w_{2}, \ldots, w_{k}\right\}}\right)(w)-L \Psi(\delta ; n) \leq \operatorname{lip}_{x}^{\mathrm{rad}} f\left(\left(\Phi_{k, i}^{x}\right)^{-1}(w)\right) \leq \operatorname{lip}$ $\left(F_{k, i}^{x} \mid \mathbf{R} \times\left\{w_{2}, \ldots, w_{k}\right\}\right)(w)+L \Psi(\delta ; n)$ for a.e $w=\left(w_{1}, \ldots, w_{k}\right) \in \Phi_{k, i}^{x}\left(C_{k, i}^{x}\right)$.

5. $(1-\Psi(\delta ; n)) \operatorname{Lip}\left(\left.F_{k, i}^{x}\right|_{\left\{w_{1}\right\} \times \mathbf{R}^{k-1}}\right)(w) \leq \operatorname{Lip}\left(\left.f\right|_{\left.\partial B_{\overline{x,\left(\Phi_{k, i}^{x}\right)^{-1}(w)}}(x) \cap C_{k, i}^{x}\right)}\right)$ $\left(\left(\Phi_{k, i}^{x}\right)^{-1}(w)\right) \leq(1+\Psi(\delta ; n)) \operatorname{Lip}\left(\left.F_{k, i}^{x}\right|_{\left.\left\{w_{1}\right\} \times \mathbf{R}^{k-1}\right)(w)}\right.$ for a.e. $w=$ $\left(w_{1}, \ldots, w_{k}\right) \in \Phi_{k, i}^{x}\left(C_{k, i}^{x}\right)$.

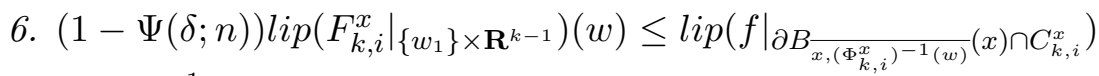

$\left(\left(\Phi_{k, i}^{x}\right)^{-1}(w)\right) \leq(1+\Psi(\delta ; n)) \operatorname{lip}\left(\left.F_{k, i}^{x}\right|_{\left.\left\{w_{1}\right\} \times \mathbf{R}^{k-1}\right)(w)}\right.$ for a.e. $w=$ $\left(w_{1}, \ldots, w_{k}\right) \in \Phi_{k, i}^{x}\left(C_{k, i}^{x}\right)$.

The proof is as follows. First, we shall give a proof of Statement 1 . Put $\mathbf{C}_{k, i}^{x}=\operatorname{Leb}\left(\Phi_{k, i}^{x}\left(C_{k, i}^{x}\right)\right) \cap \Phi_{k, i}^{x}\left(\operatorname{Leb} C_{k, i}^{x}\right)$. Then, we have $H^{k}\left(\Phi_{k, i}^{x}\left(C_{k, i}^{x}\right) \backslash\right.$ $\left.\mathbf{C}_{k, i}^{x}\right)=0$. By Lemma 3.7 and Claim 3.14 , we have $(1-\Psi(\delta))$ Lip $\left(\left.F_{k, i}^{x}\right|_{\Phi_{k, i}\left(C_{k, i}^{x}\right)}\right)(w) \leq \operatorname{Lip}\left(\left.f\right|_{C_{k, i}^{x}}\right)\left(\left(\Phi_{k, i}^{x}\right)^{-1}(w)\right) \leq(1+\Psi(\delta)) \operatorname{Lip}\left(\left.F_{k, i}^{x}\right|_{\Phi_{k, i}^{x}\left(C_{k, i}^{x}\right)}\right)$ $(w), \operatorname{Lip}\left(\left.F_{k, i}^{x}\right|_{\Phi_{k, i}^{x}\left(C_{k, i}^{x}\right)}\right)(w)=\operatorname{Lip} F_{k, i}^{x}(w)$ and $\operatorname{Lip}\left(\left.f\right|_{C_{k, i}^{x}}\right)\left(\left(\Phi_{k, i}^{x}\right)^{-1}(w)\right)=\operatorname{Lip}$ $f\left(\left(\Phi_{k, i}^{x}\right)^{-1}(w)\right)$ for every $w \in \mathbf{C}_{k, i}^{x}$. Therefore, we have statement 1 . Similarly, we have Statement 2.

Next, we shall give a proof of Statement 3. Put $\mathbf{C}_{k, i}^{x, f}=s l_{1}-\operatorname{Leb}_{k, i}^{x} \cap$ $\left\{w \in \mathbf{R}^{k} ; F_{k, i}^{x}\right.$ is totally differentiable at $\left.w\right\}$. Then, by Claim 3.15 , we have $H^{k}\left(\mathbf{C}_{k, i}^{x} \backslash \mathbf{C}_{k, i}^{x, f}\right)=0$. Fix $w \in \mathbf{C}_{k, i}^{x, f}$ and put $w_{\epsilon}=w+(\epsilon, 0, \ldots, 0)$ for every $\epsilon>0$. Since $w \in s l_{1}-\operatorname{Leb}_{k, i}^{x}$, for every $\epsilon>0$, there exist $\hat{w}_{\epsilon} \in \mathbf{C}_{k, i}^{x}$ and $a(\epsilon)>0$ such that $\overline{w_{\epsilon}, \hat{w}_{\epsilon}} \leq a(\epsilon) \epsilon$ and $a(\tau) \rightarrow 0$ as $\tau \rightarrow 0$. h is clear that (1$\delta)(\epsilon-a(\epsilon) \epsilon) \leq(1-\delta) \overline{w, \hat{w}_{\epsilon}} \leq \overline{\left(\Phi_{k, i}^{x}\right)^{-1}(w),\left(\Phi_{k, i}^{x}\right)^{-1}\left(\hat{w}_{\epsilon}\right)} \leq(1+\delta) \overline{w, \hat{w}_{\epsilon}} \leq(1$ 
$+\delta)(\epsilon+a(\epsilon) \epsilon)$. Let $\pi_{1}$ be the projection from $\mathbf{R}^{k}$ to $\mathbf{R}$ defined by $\pi_{1}(w)=$ $w_{1}$. Then we have $\overline{x,\left(\Phi_{k, i}^{x}\right)^{-1}\left(\hat{w}_{\epsilon}\right)}=\pi_{1}\left(\hat{w}_{\epsilon}\right)=\pi_{1}\left(w_{\epsilon}\right) \pm a(\epsilon) \epsilon=\pi_{1}(w)+\epsilon \pm$ $a(\epsilon) \epsilon=\overline{x,\left(\Phi_{k, i}^{x}\right)^{-1}(w)}+\overline{\left(\Phi_{k, i}^{x}\right)^{-1}(w),\left(\Phi_{k, i}^{x}\right)^{-1}\left(\hat{w}_{\epsilon}\right)} \pm(\delta+a(\epsilon)) \epsilon$. By Lemma 3.5 , without loss of generality, we can assume that there exists $\tau_{0}>0$ such that $C_{k, i} \subset \mathcal{D}_{x}^{\tau_{0}}$. Fix an isometric embedding $\gamma$ from $\left[0, \overline{x,\left(\Phi_{k, i}^{x}\right)^{-1}(w)}+\right.$ $\tau_{0}$ ] to $Y$ with $\gamma(0)=x, \gamma\left(\overline{x,\left(\Phi_{k, i}^{x}\right)^{-1}(w)}\right)=\left(\Phi_{k, i}^{x}\right)^{-1}(w)$. Then, by rescaling $\epsilon^{-1} d_{Y}$ and the splitting theorem on limit spaces, we have $\overline{\left(\Phi_{k, i}^{x}\right)^{-1}\left(\hat{w}_{\epsilon}\right), \gamma\left(\overline{x,\left(\Phi_{k, i}^{x}\right)^{-1}(w)+\epsilon}\right)} \leq \Psi(a(\epsilon), \delta ; n) \epsilon$. Thus, we have

$$
\begin{aligned}
\frac{\left|F_{k, i}^{x}(w)-F_{k, i}^{x}\left(w_{\epsilon}\right)\right|}{\epsilon} \leq & \frac{\left|F_{k, i}^{x}(w)-F_{k, i}^{x}\left(\hat{w}_{\epsilon}\right)\right|}{\epsilon}+L a(\epsilon) \\
\leq & \frac{\left|f\left(\left(\Phi_{k, i}^{x}\right)^{-1}(w)\right)-f\left(\gamma\left(\overline{x,\left(\Phi_{k, i}^{x}\right)^{-1}(w)}+\epsilon\right)\right)\right|}{\epsilon} \\
& +L \Psi(a(\epsilon), \delta ; n)
\end{aligned}
$$

for every $\epsilon>0$ with $\epsilon \ll \tau_{0}$. By letting $\epsilon \rightarrow 0$, we have $\operatorname{Lip}\left(\left.F_{k, i}^{x}\right|_{\mathbf{R} \times\left\{w_{2}, \ldots, w_{k}\right\}}\right)$ $(w) \leq \operatorname{Lip}_{x}^{\mathrm{rad}} f\left(\left(\Phi_{k, i}^{x}\right)^{-1}(w)\right)+L \Psi(\delta ; n)$. Let $\left\{\epsilon_{i}\right\}_{i}$ be a sequence of real numbers such that $\epsilon_{j} \rightarrow 0$ and

$$
\lim _{j \rightarrow \infty} \frac{\left|f \circ\left(\Phi_{k, i}^{x}\right)^{-1}(w)-f\left(\gamma\left(\overline{x,\left(\Phi_{k, i}^{x}\right)^{-1}(w)}+\epsilon_{j}\right)\right)\right|}{\left|\epsilon_{j}\right|}=\operatorname{Lip}_{x}^{\operatorname{rad}} f\left(\left(\Phi_{k, i}^{x}\right)^{-1}(w)\right)
$$

Since $\left(\Phi_{k, i}^{x}\right)^{-1}(w) \in \operatorname{Leb} C_{k, i}^{x}$, there exist sequences $\{\hat{w}(j)\}_{j} \subset C_{k, i}^{x},\left\{\tau_{j}\right\}_{j} \subset$ $\mathbf{R}_{>0}$ such that $\overline{\hat{w}(j), \gamma\left(\overline{x,\left(\Phi_{k, i}^{x}\right)^{-1}(w)}+\epsilon_{j}\right)} \leq \tau_{j} \epsilon_{j}$ and $\tau_{j} \rightarrow 0$ as $j \rightarrow \infty$. Fix $j \in \mathbf{N}$. Assume that $\epsilon_{j}>0$. Then, we have

$$
\begin{aligned}
& \pi_{1}(\hat{w}(j))-\pi_{1}(w)=\overline{x, \hat{w}(j)}-\overline{x,\left(\Phi_{k, i}^{x}\right)^{-1}(w)} \\
& =\overline{x, \gamma\left(\overline{x,\left(\Phi_{k, i}^{x}\right)^{-1}(w)}+\epsilon_{j}\right)} \pm \tau_{j} \epsilon_{j} \\
& =\epsilon_{j} \pm \tau_{j} \epsilon_{j} \\
& =\overline{\gamma\left(\overline{x,\left(\Phi_{k, i}^{x}\right)^{-1}(w)}+\epsilon_{j}\right),\left(\Phi_{k, i}^{x}\right)^{-1}(w)} \pm \tau_{j} \epsilon_{j} \\
& \geq(1-\delta) \overline{\Phi_{k, i}^{x}(\hat{w}(j)), w}-\tau_{j} \epsilon_{j} \text {. }
\end{aligned}
$$

On the other hand, since $\overline{\Phi_{k, i}^{x}(\hat{w}(j)), w} \leq(1+\delta) \epsilon_{j}+\tau_{j} \epsilon_{j}$, we have $\overline{w+\left(\epsilon_{j}, 0, \ldots, 0\right), \Phi_{k, i}^{x}(\hat{w}(j))} \leq \Psi\left(\left|\epsilon_{j}\right|, \delta ; n\right)\left|\epsilon_{j}\right|$. Similarly, we have $\overline{w+\left(\epsilon_{j}, 0, \ldots, 0\right), \Phi_{k, i}^{x}(\hat{w}(j))} \leq \Psi\left(\left|\epsilon_{j}\right|, \delta ; n\right)\left|\epsilon_{j}\right|$ in the case $\epsilon_{j}<0$. Put $w(j)=$ 
$w+\left(\epsilon_{j}, 0, \ldots, 0\right)$. Then, we have

$$
\begin{aligned}
& \frac{\mid f\left(\left(\Phi_{k, i}^{x}\right)^{-1}(w)\right)-f\left(\gamma\left(\overline{x,\left(\Phi_{k, i}^{x}\right)^{-1}(w)}+\epsilon_{j}\right)\right)}{\left|\epsilon_{j}\right|} \\
& \quad \leq \frac{\left|F_{k, i}^{x}(w)-F_{k, i}^{x}\left(\Phi_{k, i}^{x}(\hat{w}(j))\right)\right|}{\left|\epsilon_{j}\right|}+L \tau_{j} \\
& \quad \leq \frac{\left|F_{k, i}^{x}(w)-F_{k, i}^{x}(w(j))\right|}{\left|\epsilon_{j}\right|}+L \Psi\left(\left|\epsilon_{j}\right|, \tau_{j}, \delta ; n\right) .
\end{aligned}
$$

By letting $j \rightarrow \infty$, we have statement 3. Similarly, we have statement 4 .

We shall give a proof of statement 5. Fix $w \in \mathbf{C}_{k, i}^{x, f}$. By Claim 3.14, we have

$$
\begin{aligned}
(1 & -\Psi(\delta)) \operatorname{Lip}\left(\left.F_{k, i}^{x}\right|_{\left(\left\{w_{1}\right\} \times \mathbf{R}^{k-1}\right) \cap \mathbf{C}_{k, i}^{x}}\right)(w) \\
& \leq \operatorname{Lip}\left(\left.f\right|_{\left(\Phi_{k, i}^{x}\right)^{-1}}\left(\left(\left\{w_{1}\right\} \times \mathbf{R}^{k-1}\right) \cap \mathbf{C}_{k, i}^{x}\right)\right. \\
& \leq\left(\left(\Phi_{k, i}^{x}\right)^{-1}(w)\right) \\
& \leq(1+\Psi(\delta)) \operatorname{Lip}\left(\left.F_{k, i}^{x}\right|_{\left(\left\{w_{1}\right\} \times \mathbf{R}^{k-1}\right) \cap \mathbf{C}_{k, i}^{x}}\right)(w) .
\end{aligned}
$$

We remark that $\left(\Phi_{k, i}^{x}\right)^{-1}\left(\left(\left\{w_{1}\right\} \times \mathbf{R}^{k-1}\right) \cap \mathbf{C}_{k, i}^{x}\right)=\partial B \overline{x,\left(\Phi_{k, i}^{x}\right)^{-1}(w)}(x) \cap$ $\left(\Phi_{k, i}^{x}\right)^{-1}\left(\mathbf{C}_{k, i}^{x}\right)$. By Proposition 3.7, we have $\operatorname{Lip}\left(\left.F_{k, i}^{x}\right|_{\left\{w_{1}\right\} \times \mathbf{R}^{k-1} \cap \mathbf{C}_{k, i}^{x}}\right)(w)=$

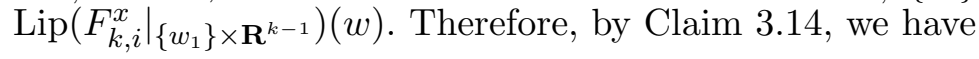

$$
\begin{aligned}
& (1-\Psi(\delta)) \operatorname{Lip}\left(\left.F_{k, i}^{x}\right|_{\left.\left\{w_{1}\right\} \times \mathbf{R}^{k-1}\right)(w)}\right. \\
& \leq \operatorname{Lip}\left(\left.f\right|_{\partial B_{\overline{x,\left(\Phi_{k, i}^{x}\right)^{-1}(w)}}(x) \cap\left(\Phi_{k, i}^{x}\right)^{-1}\left(\mathbf{C}_{k, i}^{x}\right)}\right)\left(\left(\Phi_{k, i}^{x}\right)^{-1}(w)\right) \\
& \leq \operatorname{Lip}\left(\left.f\right|_{\partial B_{\frac{x,\left(\Phi_{k, i}^{x}\right)^{-1}(w)}{x}}(x) \cap C_{k, i}^{x}}\right)\left(\left(\Phi_{k, i}^{x}\right)^{-1}(w)\right) \\
& \leq(1+\Psi(\delta)) \operatorname{Lip}\left(\left.F_{k, i}^{x}\right|_{\left(\left\{w_{1}\right\} \times \mathbf{R}^{k-1}\right) \cap \Phi_{k, i}^{x}\left(C_{k, i}^{x}\right)}\right)(w) \\
& \leq(1+\Psi(\delta)) \operatorname{Lip}\left(\left.F_{k, i}^{x}\right|_{\left\{w_{1}\right\} \times \mathbf{R}^{k-1}}\right)(w) \text {. }
\end{aligned}
$$

Thus, we have Statement 5. Similarly, we have Statement 6.

Therefore, we have Claim 3.16.

Claim 3.17. With the same notation as in Claim 3.16, we have

$$
\begin{aligned}
& \operatorname{lip}\left(\left.f\right|_{\partial B_{\overline{x,\left(\Phi_{k, i}^{x}\right)^{-1}(w)}}(x) \cap C_{k, i}^{x}}\right)\left(\left(\Phi_{k, i}^{x}\right)^{-1}(w)\right) \\
& \quad \geq \operatorname{Lip}\left(\left.f\right|_{\partial B_{\overline{x,\left(\Phi_{k, i}^{x}\right)^{-1}(w)}}(x)}\right)\left(\left(\Phi_{k, i}^{x}\right)^{-1}(w)\right)-\Psi(\delta ; n, L)
\end{aligned}
$$

for a.e $w \in \Phi_{k, i}^{x}\left(C_{k, i}^{x}\right)$. 
The proof is as follows. We shall use the same notaion as in the proof of Claim 3.16. Fix $w \in \Phi_{k, i}^{x}\left(\operatorname{Leb}\left(\Phi_{k, i}^{x}\right)^{-1}\left(\mathbf{C}_{k, i}^{x, f}\right)\right)$ and put $z=\left(\Phi_{k, i}^{x}\right)^{-1}(w)$.

First, assume $k \geq 2$. Then we shall prove that $z$ is not an isolated point in $\partial B_{\overline{x, z}}(x) \backslash C_{x}$. Because, by the definition of $s l_{1}-\operatorname{Leb}\left(\mathbf{C}_{k, i}^{x}\right)$, there exists a sequence of points $\{\beta(j)\}_{j}$ in $\mathbf{C}_{k, i}^{x}$ such that $\pi_{1}(\beta(j))=\pi_{1}(w)$, $\beta(j) \neq w$ for every $j$, and $\beta(j) \rightarrow w$. Then, we have $\left(\Phi_{k, i}^{x}\right)^{-1}(\beta(j)) \neq z$, $\left(\Phi_{k, i}^{x}\right)^{-1}(\beta(j)) \in \partial B_{\overline{x, z}}(x) \backslash C_{x}$ and $\left(\Phi_{k, i}^{x}\right)^{-1}(\beta(j)) \rightarrow z$. Especially, $z$ is not an isolated point in $\partial B_{\overline{x, z}}(x) \backslash C_{x}$. Let $\{z(j)\}_{j} \subset \partial B_{\overline{x, z}}(x) \backslash\{z\}$ with $z(j) \rightarrow$ $z,|f(z(j))-f(z)| \overline{z(j), z} \rightarrow \operatorname{Lip}\left(\left.f\right|_{\partial B_{\bar{x}, z}(x)}\right)(z)$. Put $\eta_{j}=\overline{z(j), z}>0$. Since $z \in \operatorname{Leb}\left(\Phi_{k, i}^{x}\right)^{-1}\left(\mathbf{C}_{k, i}^{x, f}\right)$, there exist sequences $\{\hat{z}(j)\}_{j} \subset\left(\Phi_{k, i}^{x}\right)^{-1}\left(\mathbf{C}_{k, i}^{x, f}\right)$ and $\left\{\hat{\tau}_{j}\right\}_{j} \mathbf{R}_{>0}$ such that $\overline{z(j), \hat{z}(j)} \leq \hat{\tau}_{j} \eta_{j}$ and $\hat{\tau}_{j} \rightarrow 0$ as $j \rightarrow \infty$. Put $\alpha(j)=$ $\Phi_{k, i}^{x}(\hat{z}(j))$. Then we have $\left|\pi_{1}(\alpha(j))-\pi_{1}(w)\right| \leq(1+\delta) \hat{\tau}_{j} \eta_{j}$. Therefore, there exists $\hat{\alpha}(j) \in\left\{w_{1}\right\} \times \mathbf{R}^{k-1}$ such that $\overline{w(j), \hat{\alpha}(j)} \leq \Psi\left(\hat{\tau}_{j} ; n\right) \eta_{j}$. Then, we have

$$
\begin{aligned}
\frac{|f(z(j))-f(z)|}{\overline{z(j), z}} & \leq \frac{|f(\hat{z}(j))-f(z)|}{\eta_{j}}+L \hat{\tau}_{j} \\
& \leq \frac{\left|F_{k, i}^{x}(w(j))-F_{k, i}^{x}(w)\right|}{\eta_{j}}+\Psi\left(\hat{\tau}_{j} ; n, L\right) \\
& \leq \frac{\left|F_{k, i}^{x}(\hat{\alpha}(j))-F_{k, i}^{x}(w)\right|}{\overline{\hat{\alpha}(j), w}} \frac{\overline{\hat{\alpha}(j), w}}{\eta_{j}}+L \Psi\left(\hat{\tau}_{j} ; n, L\right) .
\end{aligned}
$$

By letting $j \rightarrow \infty$, we have Claim 3.17 for the case $k \geq 2$.

Next, assume $k=1$. It suffices to check that $z$ is an isolated point in $\partial B_{\overline{x, z}}(x)$. The proof is done by a contradiction. Assume that $z$ is not an isolated point in $\partial B_{\overline{x, z}}(x)$. Then, there exists a sequence $\{z(i)\}_{i}$ of points in $\partial B_{\overline{x, z}}(x) \backslash\{z\}$ such that $z(i) \rightarrow z$. On the other hand, there exist $\tau_{0}>0$ and an isometric embedding $\gamma$ from $\left[0, \overline{x, z}+\tau_{0}\right]$ to $Y$ such that $\gamma(0)=x$ and $\gamma(\overline{x, z})=z$. Put $\epsilon(i)=\overline{z, z(i)}$. Then we have $\overline{z(i), \gamma\left(\overline{x, z}-\epsilon_{i}\right)} \geq \overline{x, z(i)}-$ $\overline{x, \gamma\left(\overline{x, z}-\epsilon_{i}\right)}=\epsilon_{i}$ and $\overline{z(i), \gamma\left(\overline{x, z}+\epsilon_{i}\right)} \geq \overline{x, \gamma\left(\overline{x, z}+\epsilon_{i}\right)}-\overline{x, z(i)}=\epsilon_{i}$. By Gromov's compactness theorem, without loss of generality, we can assume that $\left(Y, \epsilon_{i}^{-1} d_{Y}, z\right)$ converges to a tangent cone $\left(T_{z} Y, 0_{z}\right)$ at $z$. By the argument above and the splitting theorem on limit spaces, there exists a pointed proper geodesic space $(W, w)$ such that $T_{z} Y=\mathbf{R} \times W$ and $W \neq\{w\}$. However, since $z \in C_{1, i} \subset \mathcal{R}_{1}$, this is a contradiction. Therefore, we have the Claim 3.17.

By Claims 3.13, 3.16 and 3.17, for every $N \in \mathbf{N}$, we have $\operatorname{Lip} f(z)^{2}=$ $\operatorname{Lip}_{x}^{\mathrm{rad}} f(z)^{2}+\operatorname{Lip}\left(\left.f\right|_{\partial B_{\bar{x}, z(x)}}\right)(z)^{2} \pm N^{-1}=\operatorname{lip}_{x}^{\mathrm{rad}} f(z)^{2}+\operatorname{lip}\left(\left.f\right|_{\partial B_{\bar{x}, z}(x) \backslash C_{x}}\right)$ $(z)^{2} \pm N^{-1}=\operatorname{lipf}(z)^{2} \pm N^{-1}$ for a.e. $z \in Y \backslash C_{x}$. Therefore, we have the assertion. 
Remark 3.1. For every Ricci limit space $(Y, y, v)$ and every Lipschitz function $f$ on $Y$, we have $\operatorname{lip} f(x)=\operatorname{Lip} f(x)$ for a.e. $x \in Y$. See [2, Corollary 6.36]

By an argument similar to the proof of Lemma 3.6, we have the following:

Lemma 3.8. Let $(Y, y)$ be a Ricci limit space with $Y \neq\{y\}$, z a point in $Y \backslash$ $C_{y}, f$ a Lipschitz function on $Y, \tau$ a positive number and $\left\{\gamma_{i}\right\}_{i=1,2}$ isometric embeddings from $[0, \overline{y, z}+\tau]$ to $Y$ with $\gamma_{i}(0)=y, \gamma_{i}(\overline{y, z})=z$. Then, we have $\liminf _{r \rightarrow 0}\left|f \circ \gamma_{1}(\overline{y, z}+r)-f(z)\right| /|r|=\liminf _{r \rightarrow 0}\left|f \circ \gamma_{2}(\overline{y, z}+r)-f(z)\right| /|r|$. Moreover, if the limit $\lim _{r \rightarrow 0}\left(f \circ \gamma_{1}(\overline{y, z}+r)-f(z)\right) / r$ exists, then, we have $\lim _{r \rightarrow 0}\left(f \circ \gamma_{2}(\overline{y, z}+r)-f(z)\right) / r=\lim _{r \rightarrow 0}\left(f \circ \gamma_{1}(\overline{y, z}+r)-\right.$ $f(z)) / r$.

With the same notaion as in Lemma 3.8, put $\operatorname{Lip}_{x}^{\text {rad }} f(z)=\liminf _{r \rightarrow 0} \mid f \circ$ $\gamma_{1}(\overline{y, z}+r)-f(z)|/| r \mid$. Let $(Y, y)$ be a Ricci limit space with $Y \neq\{y\}$, and $f$ a Lipschitz function on $Y$. Put

$$
A_{y}=\left\{x \in Y \backslash C_{y} ; \text { The limit } \lim _{r \rightarrow 0} \frac{f \circ \gamma(\overline{x, y}+r)-f(x)}{r} \text { exists }\right\} .
$$

Here $\gamma$ is an isometric embedding from $[0, \overline{y, x}+\tau](\tau>0)$ to $Y$ with $\gamma(0)=$ $y, \gamma(\overline{y, x})=x$. Put

$$
\frac{d f}{d r_{y}}(x)=\lim _{r \rightarrow 0} \frac{f \circ \gamma(\overline{x, y}+r)-f(x)}{r}
$$

for every $x \in A_{y}$.

Lemma 3.9. Let $(Y, y, v)$ be a Ricci limit space, $x$ a point in $Y$ and $f$ a Lipschitz function on $Y$. Then, we have $\underline{\operatorname{Lip}}_{x}^{\mathrm{rad}} f(z)=\operatorname{Lip}_{x}^{\mathrm{rad}} f(z)$ for a.e. $z \in Y$.

Proof. We will use the same notaion as in the proof of Claim 3.16. Put $L=$ $\operatorname{Lip} f$. Let $\delta$ be a sufficiently small positive number and $C_{k, i}^{x}$ a Borel subset of $Y$ satisfying that the map $\Phi_{k, i}^{x}=\left(r_{x}, r_{x_{k, i}^{2}}, \ldots, r_{x_{k, i}^{k}}\right)$ from $C_{k, i}^{x}$ to $\mathbf{R}^{k}$, is $(1 \pm$ $\delta)$-bi-Lipschitz to the image. Fix $w \in \mathbf{C}_{k, i}^{x, f}$ and put $z=\left(\Phi_{k, i}^{x}\right)^{-1}(w)$. There exists a positive number $\tau$ and an isometric embedding $\gamma$ from $[0, \overline{x, z}+\tau]$ to $Y$ such that $\gamma(0)=x$ and $\gamma(\overline{x, z})=z$. Let $\left\{\epsilon_{i}\right\}_{i}$ be a sequence of real numbers satisfying that $\epsilon_{i} \rightarrow 0$ and $\lim _{i \rightarrow \infty}\left|f \circ \gamma\left(\overline{x, z}+\epsilon_{i}\right)-f(z)\right| /\left|\epsilon_{i}\right|=\underline{\operatorname{Lip}}_{x}^{\operatorname{rad}} f(z)$. By an argument similar to the proof of Claim 3.8, there exist sequences 
$\{\hat{w}(j)\}_{j} \subset C_{k, i}^{x}$ and $\left\{\tau_{j}\right\}_{j} \subset \mathbf{R}_{>0}$ such that $\overline{\hat{w}(j), \gamma\left(\overline{x, z}+\epsilon_{j}\right)} \leq \tau_{j}\left|\epsilon_{j}\right|, \tau_{j} \rightarrow 0$ as $j \rightarrow \infty$, and

$$
\begin{aligned}
\frac{\left|f(z)-f\left(\gamma\left(\overline{x, z}+\epsilon_{j}\right)\right)\right|}{\left|\epsilon_{j}\right|} & =\frac{\left|F_{k, i}^{x}(w)-F_{k, i}^{x}\left(\Phi_{k, i}^{x}(\hat{w}(j))\right)\right|}{\left|\epsilon_{j}\right|}-2 L \tau_{j} \\
& \geq \frac{\left|F_{k, i}^{x}(w)-F_{k, i}^{x}\left(w_{j}\right)\right|}{\left|\epsilon_{j}\right|}-\Psi\left(\tau_{j}, \delta ; n, L\right) .
\end{aligned}
$$

By letting $j \rightarrow \infty$, we have $\operatorname{Lip}_{x}^{\operatorname{rad}} f(z) \geq \operatorname{Lip}\left(\left.F_{k, i}^{x}\right|_{\mathbf{R} \times\left\{w_{2}, \ldots, w_{k}\right\}}\right)(w)-$ $\Psi(\delta ; n, L) \geq \operatorname{Lip}_{x}^{\mathrm{rad}} f(z)-\Psi(\delta ; n, L)$. Therefore, we have the assertion.

We shall state the main theorem in this subsection:

Theorem 3.3 (Radial derivatives of Lipschitz functions). Let $(Y, y, v)$ be a Ricci limit space with $Y \neq\{y\}, x$ a point in $Y$ and $f$ a Lipschitz function on $Y$. Then, we have $v\left(Y \backslash A_{x}\right)=0$ and

$$
\frac{d f}{d r_{x}}(z)=\left\langle d f, d r_{x}\right\rangle(z)
$$

for a.e. $z \in A_{x}$.

Proof. For every $w \in Y \backslash C_{x}$, there exist $\tau>0$ and an isometric embedding $\gamma$ from $[0, \overline{x, z}+\tau]$ to $Y$ such that $\gamma(0)=x$ and $\gamma(\overline{x, w})=w$. Then, by Theorem 3.2 and Lemma 3.9, for a.e. $w \in Y \backslash C_{x}$, we have

$$
\begin{aligned}
\left\langle d r_{x}, d f\right\rangle(w)= & \frac{1}{2}\left(\operatorname{Lip}\left(r_{x}+f\right)(w)^{2}-\operatorname{Lip} f(w)^{2}-\operatorname{Lip}_{x}(w)^{2}\right) \\
= & \frac{1}{2}\left(\operatorname{Lip}_{x}^{\mathrm{rad}}\left(r_{x}+f\right)(w)^{2}+\operatorname{Lip}\left(\left.\left(r_{x}+f\right)\right|_{\partial B_{\bar{x}, z}(x) \backslash C_{x}}\right)(w)^{2}\right. \\
& \left.-\operatorname{Lip}_{x}^{\mathrm{rad}} f(w)^{2}-\operatorname{Lip}\left(\left.f\right|_{\partial B_{\overline{x, z}} \backslash C_{x}}\right)(w)^{2}-1\right) \\
= & \frac{1}{2}\left(\operatorname{Lip}_{x}^{\mathrm{rad}}\left(r_{x}+f\right)(w)^{2}+\operatorname{Lip}\left(\left.f\right|_{\partial B_{\overline{x, z}}}(x) \backslash C_{x}\right)(w)^{2}\right. \\
& \left.-\operatorname{Lip}_{x}^{\mathrm{rad}} f(w)^{2}-\operatorname{Lip}\left(\left.f\right|_{\partial B_{\overline{x, z}} \backslash C_{x}}\right)(w)^{2}-1\right) \\
= & \frac{1}{2}\left(\operatorname{Lip}_{x}^{\mathrm{rad}}\left(r_{x}+f\right)(w)^{2}-\operatorname{Lip}_{x}^{\mathrm{rad}} f(w)^{2}-1\right) \\
= & \frac{1}{2}\left(\lim _{h \rightarrow 0} \frac{\left|\left(r_{x}+f\right) \circ \gamma(\overline{x, w}+h)-\left(r_{x}+f\right)(w)\right|^{2}}{|h|^{2}}\right. \\
& \left.-\lim _{h \rightarrow 0} \frac{|f \circ \gamma(\overline{x, w}+h)-f(w)|^{2}}{|h|^{2}}-1\right)
\end{aligned}
$$




$$
\begin{aligned}
= & \frac{1}{2}\left(\lim _{h \rightarrow 0}\left|1+\frac{f \circ \gamma(\overline{x, w}+h)-f(w)}{h}\right|^{2}\right. \\
& \left.-\lim _{h \rightarrow 0} \frac{|f \circ \gamma(\overline{x, w}+h)-f(w)|^{2}}{|h|^{2}}-1\right)
\end{aligned}
$$

(Here, we have the existence of the limit $\lim _{h \rightarrow 0} \frac{f \circ \gamma(\overline{x, w}+h)-f(w)}{h}$ )

$$
\begin{aligned}
= & \frac{1}{2}\left(1+2 \lim _{h \rightarrow 0} \frac{f \circ \gamma(\overline{x, w}+h)-f(w)}{h}\right. \\
& +\lim _{h \rightarrow 0} \frac{|f \circ \gamma(\overline{x, w}+h)-f(w)|^{2}}{|h|^{2}} \\
& \left.-\lim _{h \rightarrow 0} \frac{|f \circ \gamma(\overline{x, w}+h)-f(w)|^{2}}{|h|^{2}}-1\right) \\
= & \lim _{h \rightarrow 0} \frac{f \circ \gamma(\overline{x, w}+h)-f(w)}{h}=\frac{d f}{d r_{x}}(w) .
\end{aligned}
$$

\subsection{Rectifiability associated with Lipschitz functions}

In this section, we will give a generalization of Theorem 3.1. The main result in this subsection is Theorem 3.4.

Lemma 3.10. Let $\delta$ be a positive number, $\left\{\left(M_{i}, m_{i}\right)\right\}_{i}$ a sequence of $n$ dimensional complete Riemannian manifolds with $\operatorname{Ric}_{M_{i}} \geq-\delta(n-1)$, $(Y, y, v)$ a $(n,-\delta)$-Ricci limit space of $\left\{\left(M_{i}, m_{i}, \underline{\mathrm{vol}}\right)\right\}_{i}, x, x_{1}, x_{2}$ points in $Y, x(i), x_{1}(i), x_{2}(i)$ points in $M_{i}$ for every $i<\infty, \boldsymbol{b}_{1}^{i}$ a harmonic function on $B_{100}(x(i))$ for every $i<\infty$, and $\boldsymbol{b}_{1}^{\infty}$ a Lipschitz function on $B_{100}(x)$. Assume that $\overline{x, x_{1}} \geq \delta^{-1}, \overline{x, x_{2}} \geq \delta^{-1}, \overline{x, x_{1}}+\overline{x, x_{2}}-\overline{x_{1}, x_{2}} \leq \delta, x(i) \rightarrow x$, $x_{j}(i) \rightarrow x_{j}(i)$ for every $j \in\{1,2\}, \sup _{i} \mathbf{L i p} \boldsymbol{b}_{1}^{i}<\infty, \boldsymbol{b}_{1}^{i} \rightarrow \boldsymbol{b}_{1}^{\infty}$ on $B_{100}(x)$, $\left|\mathbf{b}_{1}^{i}-r_{x_{1}(i)}\right|_{L^{\infty}\left(B_{100}(x(i))\right)} \leq \delta$ and

$$
\frac{1}{\operatorname{vol} B_{100}(x(i))} \int_{B_{100}(x(i))}\left(\left|\nabla \mathbf{b}_{1}^{i}-\nabla r_{x_{1}(i)}\right|^{2}+\left|\operatorname{Hess}_{\mathbf{b}_{1}^{i}}\right|^{2}\right) d \operatorname{vol} \leq \delta
$$

Then, we have

$$
\frac{1}{v\left(B_{1}(x)\right)} \int_{B_{1}(x)}\left|d \mathbf{b}_{1}^{\infty}-d r_{x_{1}}\right|^{2} d v<\Psi(\delta ; n) .
$$


We remark that Lemma 3.10 does not follow from [2, Lemma 9.10] directly. We shall give a proof of Lemma 3.10 in the proof of the following Lemma 3.11.

Lemma 3.11. Let $\delta$ be a positive number, $\left\{\left(M_{i}, m_{i}\right)\right\}_{i}$ a sequence of $n$ dimensional complete Riemannian manifolds with $\operatorname{Ric}_{M_{i}} \geq-\delta(n-1)$, $(Y, y, v)$ a $(n,-\delta)$-Ricci limit space of $\left\{\left(M_{i}, m_{i}, \underline{\mathrm{vol}}\right)\right\}_{i}, x$ a point in $Y$, $\left\{x_{j}\right\}_{1 \leq j \leq 4}$ a collection of points in $Y$, and $\{x(i)\} \cup\left\{x_{j}(i)\right\}_{1 \leq j \leq 4}$ of points in $M_{i}$ for every $i$. Assume that $x(i) \rightarrow x, x_{j}(i) \rightarrow x_{j}$ for every $j, \overline{x, x_{j}} \geq \delta^{-1}$ for every $j, \overline{x, x_{1}}+\overline{x, x_{2}}-\overline{x_{1}, x_{2}} \leq \delta$ and $\overline{x, x_{3}}+\overline{x, x_{4}}-\overline{x_{3}, x_{4}} \leq \delta$. Then, we have

$$
\begin{aligned}
& \frac{1}{v\left(B_{1}(x)\right)} \int_{B_{1}(x)} \mid\left\langle d r_{x_{1}}, d r_{x_{3}}\right\rangle d v-\frac{1}{\operatorname{vol} B_{1}(x(i))} \\
& \times \int_{B_{1}(x(i))}\left\langle d r_{x_{1}(i)}, d r_{x_{3}(i)}\right\rangle d \operatorname{vol} \mid d v<\Psi(\delta ; n)
\end{aligned}
$$

and

$$
\begin{aligned}
& \frac{1}{\operatorname{vol} B_{1}(x(i))} \int_{B_{1}(x(i))} \mid\left\langle d r_{x_{1}(i)}, d r_{x_{3}(i)}\right\rangle-\frac{1}{v\left(B_{1}(x)\right)} \\
& \times \int_{B_{1}(x)}\left\langle d r_{x_{1}}, d r_{x_{3}}\right\rangle d v \mid d \operatorname{vol}<\Psi(\delta ; n)
\end{aligned}
$$

for every sufficiently large $i$.

Proof. First, we remark the following claim:

Claim 3.18. For every sufficiently large $i$, there exist harmonic functions $\mathbf{b}_{1}^{i}, \mathbf{b}_{3}^{i}$ on $B_{100}(x(i))$ such that $\mathbf{L i p b}_{j}^{i} \leq C(n),\left|\mathbf{b}_{j}^{i}-r_{x_{j}(i)}\right|_{L^{\infty}\left(B_{100}(x(i))\right)} \leq$ $\Psi(\delta ; n)$ and

$$
\frac{1}{\operatorname{vol} B_{100}(x(i))} \int_{B_{100}(x(i))}\left(\left|d \mathbf{b}_{j}^{i}-d r_{x_{j}(i)}\right|^{2}+\left|\operatorname{Hess}_{\mathbf{b}_{j}^{i}}\right|^{2}\right) d \operatorname{vol} \leq \Psi(\delta ; n)
$$

for every $j \in\{1,3\}$.

See for instance [2, Lemma 9.8, Lemma 9.10 and Lemma 9.13], for a proof of Claim 3.18. 
Since $C(n)\left(\left|\operatorname{Hess}_{\mathbf{b}_{1}^{i}}\right|^{2}+\left|\operatorname{Hess}_{\mathbf{b}_{3}^{i}}\right|^{2}\right)$ is an upper gradient of $\left\langle d \mathbf{b}_{1}^{i}, d \mathbf{b}_{3}^{i}\right\rangle$, by the Poincaré inequality, we have

$$
\begin{aligned}
& \frac{1}{\operatorname{vol} B_{100}(x(i))} \int_{B_{100}(x(i))} \mid\left\langle d \mathbf{b}_{1}^{i}, d \mathbf{b}_{3}^{i}\right\rangle-\frac{1}{\operatorname{vol} B_{100}(x(i))} \\
& \times \int_{B_{100}(x(i))}\left\langle d \mathbf{b}_{1}^{i}, d \mathbf{b}_{3}^{i}\right\rangle d \mathrm{vol} \mid d \mathrm{vol} \\
& \leq C(n) \sqrt{\frac{1}{\operatorname{vol} B_{100}(x(i))} \int_{B_{100}(x(i))}\left(\left|\operatorname{Hess}_{\mathbf{b}_{1}^{i}}\right|^{2}+\left|\operatorname{Hess}_{\mathbf{b}_{3}^{i}}\right|^{2}\right) d \operatorname{vol}} \leq \Psi(\delta ; n) .
\end{aligned}
$$

Therefore, we have

$$
\begin{aligned}
& \frac{1}{\operatorname{vol} B_{100}(x(i))} \int_{B_{100}(x(i))} \mid\left\langle d \mathbf{b}_{3}^{i}, d r_{x_{1}(i)}\right\rangle-\frac{1}{\operatorname{vol} B_{100}(x(i))} \\
& \times \int_{B_{100}(x(i))}\left\langle d \mathbf{b}_{3}^{i}, d r_{x_{1}(i)}\right\rangle d \operatorname{vol} \mid d \operatorname{vol} \leq \Psi(\delta ; n) .
\end{aligned}
$$

Without loss of generality, we can assume that there exist Lipschitz functions $\mathbf{b}_{1}^{\infty}, \mathbf{b}_{3}^{\infty}$ on $B_{100}(x)$ such that $\mathbf{b}_{j}^{i} \rightarrow \mathbf{b}_{j}^{\infty}$ on $B_{100}(x)$. By Theorem 3.3, there exists a Borel subset $A$ of $B_{100}(x) \backslash C_{x_{1}}$ such that $v\left(B_{100}(x) \backslash A\right)=0$ and $\lim _{h \rightarrow 0}\left(f \circ \gamma\left(\overline{x_{1}, a}+h\right)-f(a)\right) / h=\left\langle d r_{x_{1}}, d \mathbf{b}_{3}^{\infty}\right\rangle(a)$ for every $a \in A$ and every minimal geodesic $\gamma$ from $x_{1}$ to $a$. By Lusin's theorem, there exists a Borel subset $A(\delta)$ of $A$ such that $v(A \backslash A(\delta))<\delta v\left(B_{1}(x)\right)$ and that the function $\left\langle d r_{x_{1}}, d f\right\rangle$ is continuous on $A(\delta)$. Define a function $f_{\eta}^{\delta}$ on $A(\delta) \backslash B_{2 \delta}(x)$ by

$$
f_{\eta}^{\delta}(z)=\sup _{w \in C_{z}\left(\left\{x_{1}\right\}\right) \cap \bar{B}_{\eta}(z)}\left|\frac{f(z)-f(w)}{\overline{z, w}}-\left\langle d r_{x_{1}}, d f\right\rangle(z)\right|
$$

for every $0<\eta<\delta$. It is easy to check that $f_{\eta}^{\delta}$ is an upper semi-continuous function. Especially, $f_{\eta}^{\delta}$ is a Borel function. We also have $\lim _{\eta \rightarrow 0} f_{\eta}^{\delta}(a)=0$ for every $a \in A$. Thus, by Egoroff's theorem, there exists a Borel subset $X=X(\delta)$ of $A(\delta)$ such that $v(A(\delta) \backslash X(\delta))<\delta v\left(B_{1}(x)\right)$ and $\lim _{\eta \rightarrow 0}\left(\sup _{a \in X}\right.$ $\left.f_{\eta}^{\delta}(a)\right)=0$. Let $\eta=\eta(\delta)$ be a positive number satisfying that $\eta \ll \delta$, and $\sup _{a \in X} f_{\eta_{0}}^{\delta}(a)<\delta$ for every $\eta_{0} \leq \eta$. For every $i$, let $X_{i}$ be the set of points $w \in B_{1}(x(i))$ satisfying that

$$
\left|\left\langle d \mathbf{b}_{3}^{i}, d r_{x_{1}(i)}\right\rangle(w)-\frac{1}{\operatorname{vol} B_{100}(x(i))} \int_{B_{100}(x(i))}\left\langle d \mathbf{b}_{3}^{i}, d r_{x_{1}(i)}\right\rangle d \operatorname{vol}\right| \leq \Psi(\delta ; n) .
$$


Then, we have $\operatorname{vol}\left(B_{1}(x(i)) \backslash X_{i}\right) / \operatorname{vol} B_{1}(x(i)) \leq \Psi(\delta ; n)$ for every sufficiently large $i$. Define a Borel function $F_{i}$ on $B_{100}(x(i)) \backslash C_{x_{1}(i)}$ by

$$
F_{i}(w)=\frac{\mathbf{b}_{3}^{i}\left(\gamma\left(\overline{x_{1}(i), w}-\eta^{2}\right)\right)-\mathbf{b}_{3}^{i}(w)}{-\eta^{2}}
$$

for every $i$, where $\gamma$ is the minimal geodesic from $x_{1}(i)$ to $w$.

Claim 3.19. We have

$$
\frac{1}{\operatorname{vol} B_{10}(x(i))} \int_{B_{10}(x(i)) \backslash C_{x_{1}(i)}}\left|\left\langle d \boldsymbol{b}_{3}^{i}, d r_{x_{1}(i)}\right\rangle-F_{i}(w)\right| d \operatorname{vol} \leq \Psi(\delta ; n)
$$

for every sufficiently large $i$.

The proof is as follows. It is easy to check that

$$
f(t)=f(c)+f^{\prime}(t)(t-c)-\int_{c}^{t}(s-c) f^{\prime \prime}(s) d s
$$

for every $a<b$, every $C^{2}$-function $f$ on $(a, b)$, and every $c \in(a, b)$. Therefore, we have

$$
\begin{aligned}
& \frac{\mathbf{b}_{3}^{i}\left(\gamma\left(\overline{x_{1}(i), w}-\eta^{2}\right)\right)-\mathbf{b}_{3}^{i}(w)}{-\eta^{2}} \\
& \quad=\frac{d \mathbf{b}_{3}^{i}}{d r_{x_{1}(i)}}(w)-\frac{1}{\eta^{2}} \int_{\overline{x_{1}(i), w}-\eta^{2}}^{\overline{x_{1}(i), w}}\left(s-\left(\overline{x_{1}(i), w}-\eta^{2}\right)\right) \frac{d^{2} \mathbf{b}_{3}^{i}}{d r_{x_{1}(i)}^{2}}(\gamma(s)) d s .
\end{aligned}
$$

Thus, by an argument similar to the proof of [21, Estimate 2.6], we have

$$
\begin{aligned}
& \frac{1}{\operatorname{vol} B_{10}(x(i))} \int_{B_{10}(x(i)) \backslash C_{x_{1}(i)}}\left|\left\langle d \mathbf{b}_{3}^{i}, d r_{x_{1}(i)}\right\rangle-F_{i}(w)\right| d \mathrm{vol} \\
& \quad \leq \frac{1}{\eta^{2}} \frac{1}{\operatorname{vol} B_{10}(x(i))} \int_{B_{10}(x(i))} \int_{\overline{x_{1}(i), w}-\eta^{2}}^{\overline{x_{1}(i), w}} \eta^{2}\left|\operatorname{Hess}_{\mathbf{b}_{3}^{i}}\right|(\gamma(s)) d s d \underline{\mathrm{vol}} \\
& \quad \leq \eta^{2} C(n) \frac{1}{\operatorname{vol} B_{100}(x(i))} \int_{B_{100}(x(i))}^{\left|\mathrm{Hess}_{\mathbf{b}_{3}^{i}}\right| d \mathrm{vol}} \\
& \quad \leq \eta^{2} C(n) \sqrt{\frac{1}{\operatorname{vol} B_{100}(x(i))} \int_{B_{100}(x(i))}\left|\operatorname{Hess}_{\mathbf{b}_{3}^{i}}\right|^{2} d \mathrm{vol}} \leq \eta^{2} C(n) \Psi(\delta ; n) .
\end{aligned}
$$

Therefore, we have Claim 3.19 
Claim 3.20. We have

$$
\begin{aligned}
& \frac{1}{v\left(B_{1}(x)\right)} \int_{B_{1}(x)} \mid\left\langle d \boldsymbol{b}_{3}^{\infty}, d r_{x_{1}}\right\rangle \\
& \quad-\frac{1}{\operatorname{vol} B_{1}(x(i))} \int_{B_{1}(x(i))}\left\langle d \boldsymbol{b}_{3}^{i}, d r_{x_{1}(i)}\right\rangle d \operatorname{vol} \mid d v \leq \Psi(\delta ; n)
\end{aligned}
$$

for sufficiently large $i$.

The proof is as follows. Let $Y_{i}=\left\{w \in \bar{B}_{1}(x(i)) \backslash C_{x_{1}(i)} ; \mid\left\langle d \mathbf{b}_{3}^{i}, d r_{x_{1}(i)}\right\rangle\right.$ $\left.(w)-F_{i}(w) \mid \leq \Psi(\delta ; n)\right\}$. By Claim 3.19, we have vol $\left(\bar{B}_{1}(x(i)) \backslash Y_{i}\right) /$ vol $\bar{B}_{1}(x(i)) \leq \Psi(\delta ; n)$ for every sufficiently large $i$. Put $Z_{i}=X_{i} \cap Y_{i}$. There exists a compact subset $W_{i}$ of $Z_{i}$ such that $\operatorname{vol}\left(Z_{i} \backslash W_{i}\right) / \operatorname{vol} \bar{B}_{1}(x(i)) \leq$ $\Psi(\delta ; n)$. Then, we have $\operatorname{vol}\left(\bar{B}_{1}(x(i)) \backslash W_{i}\right) / \operatorname{vol} \bar{B}_{1}(x(i)) \leq \Psi(\delta ; n)$ for every sufficiently large $i$. Without loss of generality, we can assume that there exists a compact subset $W_{\infty}$ of $\bar{B}_{1}(x)$ such that $W_{j} \rightarrow W_{\infty}$. By Lemma 2.3, we have $v\left(W_{\infty}\right) / v\left(\bar{B}_{1}(x)\right) \geq 1-\Psi(\delta ; n)$. Put $E=W_{\infty} \cap X$. Then we have $v\left(\bar{B}_{1}(x) \backslash E\right) \leq \Psi(\delta ; n) v\left(\bar{B}_{1}(x)\right)$. For every $w_{i} \in W_{i}$ and every $w \in E$, let $\gamma_{w_{i}}$ be the minimal geodesic from $x_{1}(i)$ to $w_{i}$, and $\gamma_{w}$ a minimal geodesic from $x_{1}$ to $w$. Then, there exists $i_{0}$ such that $\epsilon_{i} \ll \eta$,

$$
\left|\left\langle d \mathbf{b}_{3}^{i}, d r_{x_{1}(i)}\right\rangle(w)-\frac{\mathbf{b}_{3}^{i}\left(\gamma_{i}\left(\overline{x_{1}(i), w_{i}}-\eta^{2}\right)\right)-\mathbf{b}_{3}^{i}\left(w_{i}\right)}{-\eta^{2}}\right| \leq \Psi(\delta ; n)
$$

and

$$
\left|\left\langle d \mathbf{b}_{3}^{i}, d r_{x_{1}(i)}\right\rangle\left(w_{i}\right)-\frac{1}{\operatorname{vol} B_{100}(x(i))} \int_{B_{100}(x(i))}\left\langle d \mathbf{b}_{3}^{i}, d r_{x_{1}(i)}\right\rangle d \operatorname{vol}\right| \leq \Psi(\delta ; n)
$$

for every $i \geq i_{0}$, every $w \in E$ and every $w_{i} \in W_{i}$ with $w_{i} \rightarrow w$. Now, we shall consider the rescaled metric $\eta^{-2} d_{Y}$. Since

$$
\overline{x_{1}, \phi_{i}\left(\gamma_{i}\left(\overline{x_{1}(i), w_{i}}-\eta^{2}\right)\right)} \eta^{-2} d_{Y} \geq \eta^{-1}, \overline{\phi_{i}\left(\gamma_{i}\left(\overline{x_{1}(i), w_{i}}-\eta^{2}\right)\right), w} \eta^{-2} d_{Y} \geq \eta^{-1}
$$

and

$$
\begin{aligned}
& \overline{x_{1}, \phi_{i}\left(\gamma_{i}\left(\overline{x_{1}(i), w_{i}}-\eta^{2}\right)\right)} \eta^{-2} d_{Y}+\overline{\phi_{i}\left(\gamma_{i}\left(\overline{x_{1}(i), w_{i}}-\eta^{2}\right)\right), w} \eta^{-2} d_{Y} \\
& -\bar{x}_{1}, w^{-2} d_{Y} \leq \eta
\end{aligned}
$$


by the splitting theorem on limit spaces, we have

$$
\overline{\phi_{i}\left(\gamma_{i}\left(\overline{x_{1}(i), w_{i}}-\eta^{2}\right)\right), \gamma\left(\overline{x_{1}, w}-\eta^{2}\right)} \eta^{-2} d_{Y} \leq \Psi(\delta ; n) .
$$

Therefore, we have

$$
\left|\frac{\mathbf{b}_{3}^{i}\left(\gamma_{i}\left(\overline{x_{1}(i), w_{i}}-\eta^{2}\right)\right)-\mathbf{b}_{3}^{i}\left(w_{i}\right)}{-\eta^{2}}-\frac{\mathbf{b}_{3}^{\infty}\left(\gamma\left(\overline{x_{1}, w}-\eta^{2}\right)\right)-\mathbf{b}_{3}^{\infty}(w)}{-\eta^{2}}\right| \leq \Psi(\delta ; n) .
$$

Thus, for every $i \geq i_{0}$, we have

$$
\left|\left\langle d \mathbf{b}_{3}^{\infty}, d r_{x_{1}}\right\rangle(w)-\frac{1}{\operatorname{vol} B_{100}(x(i))} \int_{B_{100}(x(i))}\left\langle d \mathbf{b}_{3}^{i}, d r_{x_{1}(i)}\right\rangle d \operatorname{vol}\right| \leq \Psi(\delta ; n) .
$$

Let

$$
C_{i}=\frac{1}{\operatorname{vol} B_{100}(x(i))} \int_{B_{100}(x(i))}\left\langle d \mathbf{b}_{3}^{i}, d r_{x_{1}(i)}\right\rangle d \mathrm{vol}
$$

Then

$$
\begin{aligned}
& \frac{1}{v\left(B_{1}(x)\right)} \int_{B_{1}(x)}\left|\left\langle d \mathbf{b}_{3}^{\infty}, d r_{x_{1}}\right\rangle-C_{i}\right| d v \\
& =\frac{1}{v\left(B_{1}(x)\right)} \int_{B_{1}(x) \backslash E}\left|\left\langle d \mathbf{b}_{3}^{\infty}, d r_{x_{1}}\right\rangle-C_{i}\right| d v \\
& \quad+\frac{1}{v\left(B_{1}(x)\right)} \int_{E}\left|\left\langle d \mathbf{b}_{3}^{\infty}, d r_{x_{1}}\right\rangle-C_{i}\right| d v \\
& \leq \frac{C(n) v\left(B_{1}(x) \backslash E\right)}{v\left(B_{1}(x)\right)}+\frac{v(E)}{v\left(B_{1}(x)\right)} \Psi(\delta ; n) \leq \Psi(\delta ; n) .
\end{aligned}
$$

Therefore, we have Claim 3.20.

Claim 3.21. We have

$$
\frac{1}{v\left(B_{1}(x)\right)} \int_{B_{1}(x)}\left|d \boldsymbol{b}_{3}^{\infty}\right|^{2} d v \leq 1+\Psi(\delta ; n) .
$$

This proof is as follows. Since

$$
\frac{1}{\operatorname{vol} B_{1}(x(i))} \int_{B_{1}(x(i))}|| d \mathbf{b}_{3}^{i}|-1| d \operatorname{vol} \leq \Psi(\delta ; n)
$$

for every sufficiently large $i$, by [1, Lemma 16.2], there exists a compact subset $K_{i}$ of $\bar{B}_{1}(x(i))$ such that $\underline{\operatorname{vol}}\left(B_{1}(x(i)) \backslash K_{i}\right) / \underline{\operatorname{vol}} B_{1}(x(i)) \leq \Psi(\delta ; n)$ and 
$\operatorname{Lip}\left(\left.\mathbf{b}_{3}^{i}\right|_{K_{i}}\right) \leq 1+\Psi(\delta ; n)$. Without loss of generality, we can assume that there exists a compact subset $K_{\infty}$ of $\bar{B}_{1}(x)$ such that $K_{i} \rightarrow K_{\infty}$. By Lemma 2.3 , we have $v\left(K_{\infty}\right) / v\left(B_{1}(x)\right) \geq 1-\Psi(\delta ; n)$. Then, we have $\operatorname{Lip}\left(\left.\mathbf{b}_{3}^{\infty}\right|_{K_{\infty}}\right) \leq$ $1+\Psi(\delta ; n)$. Put $\hat{K}_{\infty}=\operatorname{Leb} K_{\infty}$. Then by Lemma 3.7 , we have

$$
\begin{aligned}
& \frac{1}{v\left(B_{1}(x)\right)} \int_{B_{1}(x)}\left|d \mathbf{b}_{3}^{\infty}\right|^{2} d v \\
& \quad=\frac{1}{v\left(B_{1}(x)\right)} \int_{\hat{K}_{\infty}}\left|d \mathbf{b}_{3}^{\infty}\right|^{2} d v+\frac{1}{v\left(B_{1}(x)\right)} \int_{B_{1}(x) \backslash K_{\infty}}\left|d \mathbf{b}_{3}^{\infty}\right|^{2} d v \\
& \quad \leq \frac{1}{v\left(B_{1}(x)\right)} \int_{\hat{K}_{\infty}}\left(\operatorname{Lip}_{3}^{\infty}\right)^{2} d v+C(n) \frac{v\left(B_{1}(x) \backslash K_{\infty}\right)}{v\left(B_{1}(x)\right)} \\
& \quad \leq \frac{1}{v\left(B_{1}(x)\right)} \int_{\hat{K}_{\infty}}\left(\operatorname{Lip}\left(\left.\mathbf{b}_{3}^{\infty}\right|_{K_{\infty}}\right)\right)^{2} d v+\Psi(\delta ; n) \\
& \quad \leq \frac{1}{v\left(B_{1}(x)\right)} \int_{\hat{K}_{\infty}}(1+\Psi(\delta ; n)) d v+\Psi(\delta ; n) \leq 1+\Psi(\delta ; n) .
\end{aligned}
$$

Therefore, we have Claim 3.21.

Assume that $x_{1}=x_{3}$ and $x_{2}=x_{4}$. Then, by Claims 3.18, 3.20 and 3.21, we have

$$
\begin{aligned}
& \frac{1}{v\left(B_{1}(x)\right)} \int_{B_{1}(x)}\left|d \mathbf{b}_{3}^{\infty}-d r_{x_{3}}\right|^{2} d v \\
& =\frac{1}{v\left(B_{1}(x)\right)} \int_{B_{1}(x)}\left|d \mathbf{b}_{3}^{\infty}\right|^{2} d v-2 \frac{1}{v\left(B_{1}(x)\right)} \int_{B_{1}(x)}\left\langle d \mathbf{b}_{3}^{\infty}, d r_{x_{3}}\right\rangle d v \\
& \quad+\frac{1}{v\left(B_{1}(x)\right)} \int_{B_{1}(x)}\left|d r_{x_{3}}\right|^{2} d v \\
& \leq 1+\Psi(\delta ; n)-2(1-\Psi(\delta ; n))+1 \leq \Psi(\delta ; n)
\end{aligned}
$$

for every sufficiently large $i$. Therefore, we have Lemma 3.10. On the other hand, Lemma 3.11 follows from Lemma 3.10 and Claim 3.20, directly.

Corollary 3.1. Let $\left\{\left(M_{i}, m_{i}\right)\right\}_{i}$ be a sequence of $n$-dimensional complete Riemannian manifolds with $\operatorname{Ric}_{M_{i}} \geq-(n-1),(Y, y, v)$ a Ricci limit space of $\left\{\left(M_{i}, m_{i}, \underline{\mathrm{vol}}\right)\right\}_{i}, \quad \tau \quad$ a positive number, $x, x_{1}, x_{2}$ points in $Y$, $\{x(i)\}_{i},\left\{x_{1}(i)\right\}_{i},\left\{x_{2}(i)\right\}_{i}$ sequences of points $x(i), x_{1}(i), x_{2}(i)$ in $M_{i}$. Assume that $x \in \bigcap_{j=1,2}\left(\mathcal{D}_{x_{j}}^{\tau} \backslash B_{\tau}\left(x_{j}\right)\right), x(i) \rightarrow x$, and $x_{j}(i) \rightarrow x_{j}$ for every $j$. Then, 
we have

$$
\begin{aligned}
& \frac{1}{v\left(B_{r}(x)\right)} \int_{B_{r}(x)} \mid\left\langle d r_{x_{1}}, d r_{x_{2}}\right\rangle \\
& \quad-\frac{1}{\operatorname{vol} B_{r}(x(i))} \int_{B_{r}(x)}\left\langle d r_{x_{1}(i)}, d r_{x_{2}(i)}\right\rangle d \operatorname{vol} \mid d v \leq \Psi\left(r, \frac{r}{\tau} ; n\right)
\end{aligned}
$$

and

$$
\begin{aligned}
& \frac{1}{\operatorname{vol} B_{r}(x(i))} \int_{B_{r}(x(i))} \mid\left\langle d r_{x_{1}(i)}, d r_{x_{2}(i)}\right\rangle \\
& -\frac{1}{v\left(B_{r}(x)\right)} \int_{B_{r}(x)}\left\langle d r_{x_{1}}, d r_{x_{2}}\right\rangle d v \mid d \operatorname{vol} \leq \Psi\left(r, \frac{r}{\tau} ; n\right)
\end{aligned}
$$

for every sufficiently large $i$.

Proof. By rescaling $r^{-1} d_{Y}$ and Lemma 3.11, it is easy to check the assertion.

Lemma 3.12. Let $\left\{\left(M_{i}, m_{i}\right)\right\}_{i}$ be a sequence of $n$-dimensional complete Riemannian manifolds with $\operatorname{Ric}_{M_{i}} \geq-(n-1),(Y, y, v)$ a Ricci limit space of $\left\{\left(M_{i}, m_{i}, \underline{\mathrm{vol}}\right)\right\}_{i}, l$ a positive integer, $r, \epsilon, \tau, L$ positive real numbers, $x$ a point in $Y,\{x(i)\}_{i}$ a sequence of points $x_{i}$ in $M_{i},\left\{k_{\alpha}\right\}_{1 \leq \alpha \leq l}$ a collection of positive integers, $\left\{x_{t}^{s}\right\}_{1 \leq s \leq l, 1 \leq t \leq k_{s}}$ of points in $Y,\left\{x_{t}^{s}(\bar{i})\right\}_{1 \leq s \leq l, 1 \leq t \leq k_{s}}$ of points in $M_{i}$ for every $i<\infty$, and $\left\{a_{t}^{s}\right\}_{1 \leq s \leq l, 1 \leq t \leq k_{s}}$ of real numbers. Let $f_{j}=\sum_{m=1}^{k_{j}} a_{m}^{j} r_{x_{m}^{j}}$ and $f_{j}^{i}=\sum_{m=1}^{k_{j}} a_{m}^{j} r_{x_{m}^{j}(i)}$. Assume that $l \leq n, k_{i} \leq n$ for every $1 \leq i \leq l, x \in \bigcap_{1 \leq i \leq l, 1 \leq j \leq k_{i}}^{k}\left(\mathcal{D}_{x_{j}^{i}}^{\tau} \backslash B_{\tau}\left(x_{j}^{i}\right)\right), x(i) \rightarrow x, x_{t}^{s}(i) \rightarrow x_{t}^{s}$, $\sum_{i, j}\left(a_{j}^{i}\right)^{2} \leq L$ and

$$
\frac{1}{v\left(B_{r}(x)\right)} \int_{B_{r}(x)}\left\langle d f_{j}, d f_{i}\right\rangle d v=\delta_{i j} \pm \epsilon
$$

Then, for every sufficiently large $i$, there exists a compact subset $K_{r}^{i}$ of $\bar{B}_{r / 10}(x(i))$ such that the following properties hold:

1. $\operatorname{vol}\left(B_{r / 10}(x(i)) \backslash K_{r}^{i}\right) / \operatorname{vol} B_{r / 10}(x(i)) \leq \Psi(r, r / \tau, \epsilon ; n, L)$.

2. For every $w \in K_{r}^{i}$ and every $0<s<r / 10^{6}$, there exist a compact subset $Z$ of $\bar{B}_{s}(w)$, a point $z$ in $Z$, and a map $\phi$ from $\left(\bar{B}_{s}(w), w\right)$ to $(Z, z)$ such that the map $\Phi=\left(f_{1}^{i}, f_{2}^{i}, \ldots, f_{l}^{i}, \phi\right)$ from $\bar{B}_{s}(w)$ to $\bar{B}_{s+\Psi(r, r / \tau, \epsilon ; n, L) s} \quad\left(f_{1}^{i}(w), \ldots, f_{l}^{i}(w), \phi(w)\right), \quad$ is $\quad$ a $\Psi(r, r / \tau, \epsilon ; n, L) s-$ Gromov-Hausdorff approximation. 
3. We have

$$
\frac{1}{\operatorname{vol} B_{s}(w)} \int_{B_{s}(w)}\left|\left\langle d f_{\alpha}^{i}, d f_{\beta}^{i}\right\rangle-\delta_{\alpha \beta}\right| d \underline{\operatorname{vol}}<\Psi\left(r, \frac{r}{\tau}, \epsilon ; n, L\right)
$$

for every $w \in K_{r}^{i}$ and every $0<s<r / 10^{6}$.

Proof. By Corollary 3.1, we have

$$
\frac{1}{\operatorname{vol} B_{r}(x(i))} \int_{B_{r}(x(i))}\left|\left\langle d f_{j}^{i}, d f_{\hat{l}}^{i}\right\rangle-\delta_{j, \hat{l}}\right| d \operatorname{vol} \leq \Psi\left(r, \frac{r}{\tau}, \epsilon ; n, L\right)
$$

for every sufficiently large $i$. We shall consider rescaled distances $r^{-1} d_{Y}$ and $r^{-1} d_{M_{i}}$ below. For convenience, we shall use the following notations: $\hat{\mathrm{vol}}=\mathrm{vol}^{r^{-1}} d_{M_{i}}, \hat{v}=v / v\left(B_{r}(y)\right), \hat{r}_{z}(w)=r^{-1} \overline{w, z} d_{Y}, \hat{B}_{s}(w)=B_{s}^{r^{-1}} d_{Y}(w)=$ $B_{s r}(w), \hat{g}=r^{-1} g$ for a Lipschitz function $g$ and so on. We remark that $\left(M_{i}, m_{i}, r^{-1} d_{M_{i}}, \underline{\mathrm{vol}}^{r^{-1}} d_{M_{i}}\right) \rightarrow\left(Y, y, r^{-1} d_{Y}, \hat{v}\right)$. We also denote the differential of a Lipschitz function $f$ on $Y$ as a metric measure space $(Y, \hat{v})$ by $\hat{d} f: Y \rightarrow T^{*} Y$, and the Riemannian metric of rescaled Ricci limit space $\left(Y, y, r^{-1} d_{Y}, \hat{v}\right)$ by $\langle\cdot, \cdot\rangle_{r}$. Thus, we have $\langle\cdot, \cdot\rangle_{r}=r^{-2}\langle\cdot, \cdot\rangle$. Then, we have

$$
\frac{1}{\operatorname{vol} \hat{B}_{1}(x(i))} \int_{\hat{B}_{1}(x(i))}\left|\left\langle\hat{d} \hat{f}_{j}^{i}, \hat{d} \hat{f}_{\hat{l}}^{i}\right\rangle_{r}-\delta_{j, \hat{l}}\right| d \hat{\operatorname{vol}} \leq \Psi\left(r, \frac{r}{\tau}, \epsilon ; n, L\right)
$$

for every sufficiently large $i$. On the other hand, by [2, Lemmas 9. 8, 9.10, 9.13], for every sufficiently large $i$, there exists a collection of harmonic functions $\left\{\hat{\mathbf{b}}_{j}^{m, i}\right\}_{1 \leq m \leq l, 1 \leq j \leq k_{m}}$ on $\hat{B}_{100}(x(i))$ such that $\left|\hat{\mathbf{b}}_{j}^{m, i}-\hat{r}_{x_{j}^{m}(i)}\right|_{L^{\infty}\left(\hat{B}_{100}(x(i))\right)} \leq$ $\Psi(r, r / \tau ; n)$ and

$$
\begin{aligned}
& \frac{1}{\hat{\operatorname{vol}} \hat{B}_{100}(x(i))} \int_{\hat{B}_{100}(x(i))}\left(\left|\hat{d} \hat{\mathbf{b}}_{j}^{m, i}-\hat{d} \hat{r}_{x_{j}^{m}(i)}\right|_{r}^{2}+\left|\operatorname{Hess}_{\hat{\mathbf{b}}_{j}^{m, i}}\right|_{r}^{2}\right) d \hat{\operatorname{vol}} \\
& \quad \leq \Psi\left(r, \frac{r}{\tau} ; n\right) .
\end{aligned}
$$

Let $\hat{\mathbf{b}}_{j}^{i}=\sum_{m=1}^{k_{j}} a_{m}^{j} \hat{\mathbf{b}}_{j}^{m, i}$

$$
\hat{F}_{i}=\sum_{j=1}^{l}\left|\hat{d} \hat{\mathbf{b}}_{j}^{i}-\hat{d} \hat{f}_{j}^{i}\right|_{r}^{2}+\left.\sum_{j=1}^{l}|| \hat{d}_{\mathbf{b}_{j}^{i}}^{i}\right|_{r} ^{2}-\left.1\left|+\sum_{j<\hat{l}}\right|\left\langle\hat{d} \mathbf{b}_{j}^{i}, \hat{d} \mathbf{b}_{\hat{l}}^{i}\right\rangle_{r}\left|+\sum_{j=1}^{l}\right| \operatorname{Hess}_{\hat{\mathbf{b}}_{j}^{i}}\right|_{r} ^{2}
$$

The next claim follows from Lemma 3.1, directly: 
Claim 3.22. For every sufficiently large $i$, there exists a compact subset $K_{r}^{i}$ of $\hat{B}_{1 / 10}(x(i))$ such that $\hat{\operatorname{vol}}\left(\hat{B}_{\frac{1}{10}}(x(i)) \backslash K_{r}^{i}\right) / \hat{\operatorname{vol}} \hat{B}_{\frac{1}{10}}(x(i)) \leq \Psi(r, r / \tau$, $\epsilon ; n, L)$ and

$$
\frac{1}{\hat{\operatorname{vol}} \hat{B}_{5 s}(w)} \int_{\hat{B}_{5 s}(w)} \hat{F}_{i} d \hat{\operatorname{vol}} \leq \Psi\left(r, \frac{r}{\tau}, \epsilon ; n, L\right)
$$

for every $w \in K_{r}^{i}$ and every $0<s<1 / 10$.

Fix $w \in K_{r}^{i}$ and $0<s \leq 1 / 10$. By an argument same to the proof of $[6$, Theorem 3.3], we have the following:

Claim 3.23. There exist a compact subset $Z$ of $\hat{\bar{B}}_{s}(w)$, a point $z$ in $Z$ and a map $\phi$ from $\hat{\bar{B}}_{s / 10^{5}}(w)$ to $Z$ such that the map $\Phi(\alpha)=\left(\hat{\mathbf{b}}_{1}^{i}(\alpha), \ldots, \hat{\mathbf{b}}_{l}^{i}(\alpha)\right.$, $\phi(\alpha))$ from $\hat{\bar{B}}_{s / 10^{5}}(w)$ to $\bar{B}_{s / 10^{5}+\Psi s}\left(\hat{\mathbf{b}}_{1}^{i}(w), \ldots, \hat{\mathbf{b}}_{l}^{i}(w), \phi(w)\right) \subset \mathbf{R}^{k} \times Z$, is a $\Psi$ s-Gromov-Hausdorff approximation. Here, $\Psi=\Psi(r, r / \tau, \epsilon ; n, L)$.

Since

$$
\frac{1}{\operatorname{vol} \hat{B}_{5 s}(w)} \int_{\hat{B}_{5 s}(w)}\left|\hat{d} \hat{\mathbf{b}}_{j}^{i}-\hat{d} \hat{f}_{j}^{i}\right|_{r}^{2} d \hat{\mathrm{vol}} \leq \Psi\left(r, \frac{r}{\tau}, \epsilon ; n, L\right),
$$

by the segment inequality on manifolds [6, Theorem 2.15], for every $z_{1} \in$ $\hat{\bar{B}}_{s}(w)$, there exist $\hat{z}_{1} \in \hat{\bar{B}}_{5 s}(w), \hat{w} \in \hat{\bar{B}}_{5 s}(w)$ and a minimal geodesic $\gamma$ from $\hat{z}_{1}$ to $\hat{w}$ such that $\overline{z_{1}, \hat{z}_{1}} \leq \Psi(r, r / \tau, \epsilon ; n, L), \overline{w, \hat{w}} \leq \Psi(r, r / \tau, \epsilon ; n, L)$ and

$$
\int_{0}^{\overline{\hat{z}_{1}, \hat{w}}} \operatorname{Lip}\left(\hat{\mathbf{b}}_{j}^{i}-\hat{f}_{j}^{i}\right)(\gamma(t)) d t \leq \Psi\left(r, \frac{r}{\tau}, \epsilon ; n, L\right) s .
$$

Therefore, we have

$$
\begin{aligned}
\left|\hat{\mathbf{b}}_{j}^{i}\left(\hat{z}_{1}\right)-\hat{f}_{j}^{i}\left(\hat{z}_{1}\right)-\left(\hat{\mathbf{b}}_{j}^{i}(\hat{w})-\hat{f}_{j}^{i}(\hat{w})\right)\right| & \leq \int_{0}^{\overline{\hat{z}_{1}, \hat{w}}} \operatorname{Lip}\left(\hat{\mathbf{b}}_{j}^{i}-\hat{f}_{j}^{i}\right)(\gamma(t)) d t \\
& \leq \Psi\left(r, \frac{r}{\tau}, \epsilon ; n, L\right) s
\end{aligned}
$$

By Cheng-Yau's gradient estimate, we have $\mathbf{L} \hat{\mathbf{i}} \mathbf{p}\left(\left.\hat{\mathbf{b}}_{j}^{i}\right|_{\hat{B}_{2 s}(w)}\right) \leq C(n, L)$. Thus, we have $\left|\hat{\mathbf{b}}_{j}^{i}\left(z_{1}\right)-\hat{f}_{j}^{i}\left(z_{1}\right)-\left(\hat{\mathbf{b}}_{j}^{i}(w)-\hat{f}_{j}^{i}(w)\right)\right| \leq \Psi(r, r / \tau, \epsilon ; n, L) s$. Let $C=$ $\hat{\mathbf{b}}_{j}^{i}(w)-\hat{f}_{j}^{i}(w)$. Then we have that $\hat{\mathbf{b}}_{j}^{i}=\hat{f}_{j}^{i}+C \pm \Psi(r, r / \tau, \epsilon ; n, L) s$ on $\hat{\bar{B}}_{s}(w)$. 
Thus, the map $\hat{\Phi}(\alpha)=\left(\hat{f}_{1}^{i}(\alpha), \ldots, \hat{f}_{l}^{i}(\alpha), \phi(\alpha)\right)$ from $\hat{\bar{B}}_{s / 10^{5}}(w)$ to $\bar{B}_{s / 10^{5}+\Psi s}\left(\hat{f}_{1}^{i}(w), \ldots, \hat{f}_{l}^{i}(w), \phi(w)\right)$, is a $\Psi s$-Gromov-Hausdorff approximation. Therefore, we have the assertion.

Lemma 3.13. Let $(Y, y, v)$ be a Ricci limit space, $\tau, \epsilon, \delta, L$ positive numbers, $l, m$ positive integers, $x$ a point in $Y,\left\{k_{s}\right\}_{1 \leq s \leq l}$ a collection of positive integers, $\left\{a_{t}^{s}\right\}_{1 \leq s \leq l, 1 \leq t \leq k_{s}}$ of real numbers, and $\left\{x_{t}^{s}\right\}_{1 \leq s \leq l, 1 \leq t \leq k_{s}}$ of points in $Y$. Let $f_{j}=\sum_{p=1}^{k_{j}} a_{p}^{j} r_{x_{p}^{j}}$. Assume that $x \in \operatorname{Leb}\left(\bigcap_{1 \leq i \leq l, 1 \leq j \leq k_{i}}\left(\mathcal{D}_{x_{j}^{i}}^{\tau} \backslash\left\{x_{j}^{i}\right\}\right) \cap\right.$ $\left.\left(\mathcal{R}_{m}\right)_{\delta, \tau}\right), \sum_{i, j}\left(a_{j}^{i}\right)^{2} \leq L$ and

$$
\limsup _{r \rightarrow 0} \frac{1}{v\left(B_{r}(x)\right)} \int_{B_{r}(x)}\left|\left\langle d f_{j}, d f_{i}\right\rangle-\delta_{i j}\right| d v \leq \epsilon .
$$

Then, for every sufficiently small $s>0$, there exists a compact subset $K_{s}$ of $\bar{B}_{s}(x)$ such that the following properties hold:

1. $v\left(K_{s}\right) / v\left(B_{s}(x)\right) \geq 1-\Psi(\epsilon, \delta ; n, L)$.

2. For every $\alpha \in K_{s}$ and every sufficiently small $t>0$, there exists a collection of points $\left\{w_{j}^{t}(\alpha)\right\}_{1 \leq j \leq m-l}$ in $Y$, and a compact subset $U_{t}$ of $\bar{B}_{t}(\alpha)$ such that $v\left(U_{t}\right) / v\left(B_{t}(\alpha)\right) \geq 1-\Psi(\epsilon, \delta ; n, L)$ and that the map $\Phi_{t}=\left(f_{1}, \ldots, f_{l}, r_{w_{1}^{t}(\alpha)}, \ldots, r_{w_{m-l}^{t}(\alpha)}\right)$ from $U_{t}$ to $\mathbf{R}^{m}$, is $(1 \pm \Psi(\epsilon, \delta$; $n, L)$ )-bi-Lipschitz to the image.

Proof. Let $\left(M_{i}, m_{i}, x_{t}^{s}(i), \underline{\mathrm{vol}}\right) \rightarrow\left(Y, y, x_{t}^{s}, v\right)$ and $f_{j}^{i}=\sum_{p=1}^{k_{j}} a_{p}^{j} r_{x_{p}^{j}(i)}$. There exists $s_{1}>0$ such that $s_{1} \ll \tau$ and

$$
\begin{aligned}
& \frac{1}{v\left(B_{10^{10} s}(x)\right)} \int_{B_{10^{10_{s}}}(x)}\left|\left\langle d f_{j}, d f_{i}\right\rangle-\delta_{i j}\right| d v \\
& +\frac{v\left(B_{10^{10} s}(x) \cap \bigcap_{1 \leq i \leq l, 1 \leq j \leq k_{i}}\left(\mathcal{D}_{x_{j}^{i}}^{\tau} \cap\left(\mathcal{R}_{m}\right)_{\delta, r}\right)\right)}{v\left(B_{10^{10} s}(x)\right)} \leq 3 \epsilon
\end{aligned}
$$

for every $0<s<s_{1}$. By Proposition 2.3 and Lemma 3.12, for every $0<s<$ $s_{1}$, there exists a compact subset $K_{s}$ of $\bar{B}_{10^{9} s}(x)$ such that the following properties hold:

1. $v\left(K_{s}\right) / v\left(B_{10^{9} s}(x)\right) \geq 1-\Psi(\epsilon ; n, L)$.

2. For every $w \in K_{s}$ and every $0<t<10^{4} s$, there exist a compact subset $Z_{t}^{w}$ of $\bar{B}_{t}(w)$ and a map $\phi_{t}^{w}$ from $\bar{B}_{t}(w)$ to $Z_{t}^{w}$ such that the map $\Phi_{t}^{w}=$ $\left(f_{1}, \ldots, f_{l}, \phi_{t}^{w}\right)$ from $\bar{B}_{t}(w)$ to $\bar{B}_{10^{9}(t+\Psi t)}\left(f_{1}(w), \ldots, f_{l}(w), \phi_{t}^{w}(w)\right)$, is a $\Psi t$-Gromov-Hausdorff approximation. Here $\Psi=\Psi(\epsilon ; n, L)$. 
3. We have

$$
\frac{1}{v\left(B_{t}(w)\right)} \int_{B_{t}(w)}\left|\left\langle d f_{j}, d f_{i}\right\rangle-\delta_{i j}\right| d v \leq \Psi(\epsilon ; n, L)
$$

for every $w \in K_{s}$ and every $0<t<10^{4} s$.

Here, with the same notation as in Lemma 3.12, we applied Proposition 4.7 to obtain

$$
\begin{aligned}
\lim _{k \rightarrow \infty} \frac{1}{\underline{\operatorname{vol}} B_{t}(w(k))} \int_{B_{t}(w(k))}\left|\left\langle d f_{j}^{k}, d f_{i}^{k}\right\rangle-\delta_{i j}\right| d \underline{\text { vol }} \\
\quad=\frac{1}{v\left(B_{t}(w)\right)} \int_{B_{t}(w)}\left|\left\langle d f_{j}, d f_{i}\right\rangle-\delta_{i j}\right| d v
\end{aligned}
$$

for every sequence $w(k) \rightarrow w$. Fix $0<s<s_{1}, w \in K_{s} \cap \operatorname{Leb}\left(\bigcap_{1 \leq i \leq l, 1 \leq j \leq k_{i}}\right.$ $\left.\left(\mathcal{D}_{x_{j}^{i}}^{\tau} \backslash\left\{x_{j}^{i}\right\}\right) \cap\left(\mathcal{R}_{m}\right)_{\delta, r}\right), 0<t<10^{4} s, Z_{t}^{w}, \phi_{t}^{w}$ and $\Phi_{t}^{w}$ as above. We remark that $v\left(K_{s} \cap \operatorname{Leb}\left(\bigcap_{1 \leq i \leq l, 1 \leq j \leq k_{i}}\left(\mathcal{D}_{x_{j}^{i}}^{\tau} \backslash\left\{x_{j}^{i}\right\}\right) \cap\left(\mathcal{R}_{m}\right)_{\delta, r}\right)\right) / v\left(B_{10^{9} s}(x)\right) \geq 1-$ $\Psi(\epsilon ; n, L)$. Assume that $t$ is sufficiently small and

$$
\frac{v\left(B_{\hat{t}}(w) \cap \bigcap_{1 \leq i \leq l, 1 \leq j \leq k_{i}}\left(\mathcal{D}_{x_{j}^{i}}^{\tau} \backslash\left\{x_{j}^{i}\right\}\right) \cap\left(\mathcal{R}_{m}\right)_{\delta, r}\right)}{v\left(B_{\hat{t}}(w)\right)} \geq 1-\epsilon
$$

for every $0<\hat{t} \leq t$, below. Then, for every $1 \leq j \leq l$, there exist points $y_{j}^{+}, y_{j}^{-} \in \bar{B}_{t}(w)$ such that $\overline{\Phi_{t}^{w}\left(y_{j}^{+}\right),(\underbrace{0, \ldots, 0, t}_{j}, 0, \ldots, 0, \phi_{t}^{w}(w))} \leq \Psi t$ and $\overline{\Phi_{t}^{w}\left(y_{j}^{-}\right),(\underbrace{0, \ldots, 0,-t}_{j}, 0, \ldots, 0, \phi_{t}^{w}(w))} \leq \Psi t$. Let $\hat{\Phi}_{t}^{w} \quad$ be a $\Psi t$-GromovHausdorff approximation from $\bar{B}_{10^{9}(t+\Psi t)}\left(f_{1}(w), \ldots, f_{l}(w), \phi_{t}^{w}(w)\right)$ to $\bar{B}_{t}(w)$ satisfying that $\overline{\Phi_{t}^{w} \circ \hat{\Phi}_{t}^{w}(\alpha), \alpha} \leq \Psi t \quad$ for $\quad$ every $\quad \alpha \in \bar{B}_{10^{9}(t+\Psi t)}$ $\left(f_{1}(w), \ldots, f_{l}(w), \phi_{t}^{w}(w)\right)$, and that $\overline{\hat{\Phi}_{t}^{w} \circ \Phi_{t}^{w}(\beta), \beta} \leq \Psi t$ for every $\beta \in \bar{B}_{t}(w)$. On the other hand, there exist $\delta t$-Gromov-Hausdorff approximations $\psi_{t}^{w}$ from $\left(\bar{B}_{t}(w), w\right)$ to $\left(\bar{B}_{t}\left(0_{m}\right), 0_{m}\right)$, and $\hat{\psi}_{t}^{w}$ from $\left(\bar{B}_{t}\left(0_{m}\right), 0_{m}\right)$ to $\left(\bar{B}_{t}(w), w\right)$ such that $\overline{\psi_{t}^{w} \circ \hat{\psi}_{t}^{w}(\alpha), \alpha} \leq 5 \delta t$ for every $\alpha \in \bar{B}_{t}\left(0_{m}\right)$, and that $\hat{\psi}_{t}^{w} \circ \psi_{t}^{w}(\beta), \beta$ $\leq 5 \delta t$ for every $\beta \in \bar{B}_{t}(w)$. Especially, there exists a $\Psi t$-Gromov-Hausdorff approximation $\hat{h}_{t}^{w}$ from $\left(\bar{B}_{t}\left(0_{m-l}\right), 0_{m-l}\right)$ to $\left(Z_{t}^{w}, \phi_{t}^{w}(w)\right)$ such that $\overline{(0, \ldots, 0, \alpha), \psi_{t}^{w} \circ \hat{\Phi}_{t}^{w}\left(f_{1}(w), \ldots, f_{l}(w), \hat{h}_{t}^{w}(\alpha)\right)} \leq \Psi t$ for every $\alpha \in Z_{t}^{w}$, where $\Psi=\Psi(\epsilon, \delta ; n, L)$. Without loss of generality, we can assume that $\overline{\psi_{t}^{w}\left(y_{i}^{+}\right),(\underbrace{0, \ldots, 0, t}_{i}, 0, \ldots, 0)} \leq \Psi t$. Then, for every $i \in\{l+1, \ldots, m\}$, there 
$\frac{\text { exist }}{\psi_{t}^{w}\left(z_{i}^{+}\right),(\underbrace{0, \ldots, 0, t}_{i}, 0, \ldots, 0)} \leq \Psi t$ and $z_{i}^{+}, z_{i}^{-} \in \bar{B}_{t}(w) \quad$ such $\quad$ that Let $F_{i}=f_{i}-f_{i}(w)$ and $G_{i}=F_{i} \circ \psi_{t}^{w} \quad$ on $\quad\left(\bar{B}_{t}\left(0_{m}\right), 0_{m}\right)$. Since $\pi_{\mathbf{R}^{m-l}}\left(\psi_{t}^{w} \circ \hat{\Phi}_{t}^{w}\left(f_{1}(w), \ldots, f_{l}(w), \hat{h}_{t}^{w}(\alpha)\right)\right), \alpha \leq \Psi t$ for every $\alpha \in \bar{B}_{t}\left(0_{m-l}\right)$, we have that the map $G=\left(G_{1}, \ldots, G_{l}, \pi_{l+1}, \ldots, \pi_{m}\right)$ from $\left(\bar{B}_{t}\left(0_{m}\right), 0_{m}\right)$ to $\left(\bar{B}_{t+\Psi t}\left(0_{m}\right), 0_{m}\right)$, satisfies $\overline{G((\underbrace{0, \ldots, 0, \pm t}_{i}, 0, \ldots, 0),(\underbrace{0, \ldots, 0, \pm t}_{i}, 0, \ldots, 0)} \leq$ $\Psi t$ for every $i$, and that it is a $\Psi t$-Gromov-Hausdorff approximation, where $\pi_{\mathbf{R}^{m-l}}$ is the canonical projection $\mathbf{R}^{m}=\mathbf{R}^{l} \times \mathbf{R}^{m-l}$ to $\mathbf{R}^{m-l}, \pi_{i}$ is the $i$ th projection from $\mathbf{R}^{m}$ to $\mathbf{R}$. Thus, we have $\overline{\alpha, G(\alpha)} \leq \Psi t$ for every $\alpha \in \bar{B}_{t}\left(0_{m}\right)$. Especially, we have the following claim:

Claim 3.24. We have $\left|G_{i}-\pi_{i}\right| \leq \Psi(\epsilon, \delta ; n, L)$ t on $B_{t}\left(0_{m}\right)$.

Fix $0<\hat{t}<t$. By rescaling $\hat{t}^{-1} d_{Y}, \hat{t}^{-1} d_{\mathbf{R}^{m}}$, Claim 3.24 and the definition of Busemann function, we have the following:

Claim 3.25. We have

$$
\left|F_{i}(\alpha)-\left(r_{y_{i}^{-}}(\alpha)-r_{y_{i}^{-}}(w)\right)\right| \leq \Psi\left(\epsilon, \delta, \frac{\hat{t}}{t}, \frac{\Psi(\epsilon, \delta ; n, L) t}{\hat{t}} ; n, L\right) \hat{t}
$$

for every $\alpha \in \bar{B}_{\hat{t}}(w)$.

Let $y_{j}^{-}(k), z_{j}^{-}(k), w(k)$ be points in $M_{k}$ satisfying that $y_{j}^{-}(k) \rightarrow y_{j}^{-}$, $z_{j}^{-}(k) \rightarrow z_{j}^{-}$and $w(k) \rightarrow w$. Put $r=\sqrt{\Psi} t$ for $\Psi=\Psi(\epsilon, \delta ; n, L)$ as in Claim 3.25. For convenience, for rescaled distances $r^{-1} d_{Y}$ and $r^{-1} d_{M_{i}}$, we shall use the same notation as in the proof of Lemma 3.12: $\hat{f}_{i}^{k}, \hat{d} f$, vol and so on.

Claim 3.26. We have

$$
\frac{1}{\hat{\operatorname{vol}} \hat{B}_{100}(w(k))} \int_{\hat{B}_{100}(w(k))}\left|\hat{d} \hat{f}_{i}^{k}-\hat{d} \hat{r}_{y_{i}^{-}(k)}\right|_{r}^{2} d \hat{\text { vol }} \leq \Psi(\epsilon, \delta ; n, L)
$$

for every sufficiently large $k$.

The proof is as follows. By the assumption and Proposition 4.7, we have

$$
\frac{1}{\hat{\operatorname{vol}} \hat{B}_{1000}(x(k))} \int_{\hat{B}_{1000}(x(k))} \|\left.\hat{d} \hat{f}_{i}^{k}\right|_{r} ^{2}-1 \mid d \hat{\operatorname{vol}} \leq \Psi(\epsilon, \delta ; n, L)
$$


for every sufficiently large $k$. By an argument similar to the proof of Lemma 3.12 , for every sufficiently large $k$, there exists a harmonic function $\hat{\mathbf{b}}_{i}^{k}$ on $\hat{B}_{100}(w(k))$ such that $\operatorname{Lip} \hat{\mathbf{b}}_{i}^{k} \leq C(n),\left|\hat{\mathbf{b}}_{i}^{k}-\hat{f}_{i}^{k}\right|_{L^{\infty}\left(\hat{B}_{100}(w(k))\right)} \leq \Psi(r, r /$ $\tau ; n, L)$ and

$$
\begin{aligned}
& \frac{1}{\hat{\operatorname{vol}} \hat{B}_{1000}(w(k))} \int_{\hat{B}_{1000}(w(k))}\left(\left|\hat{d} \hat{\mathbf{b}}_{i}^{k}-\hat{d} \hat{f}_{i}^{k}\right|_{r}^{2}+\left|\operatorname{Hess}_{\hat{\mathbf{b}}_{i}^{k}}\right|_{r}^{2}\right) d \hat{\operatorname{vol}} \\
& \quad \leq \Psi(r, r / \tau ; n, L) .
\end{aligned}
$$

For every $\alpha \in \hat{B}_{1000}(w(k)) \backslash C_{y_{i}^{-}(k)}$, let $\gamma_{i}^{\alpha}$ be the minimal geodesic from $y_{i}^{-}(k)$ to $\alpha$ on $\left(M_{i}, r^{-1} d_{M_{i}}\right)$. Fix $0<h<1$. By Claim 3.25, there exists $k_{0}$ such that

$$
\begin{aligned}
& \hat{\mathbf{b}}_{i}^{k}(\alpha)-\hat{\mathbf{b}}_{i}^{k}\left(\gamma_{i}^{\alpha}\left({\overline{y_{i}^{-}(k), \alpha}}^{r^{-1} d_{M_{k}}}-h\right)\right) \\
& =\frac{\hat{f}_{i}^{k}(\alpha)-\hat{f}_{i}^{k}\left(\gamma_{i}^{\alpha}\left({\overline{y_{i}^{-}(k), \alpha}}^{-1} d_{M_{k}}-h\right)\right)}{h} \pm \frac{\Psi(\epsilon, \delta ; n, L)}{h} \\
& =\frac{\hat{f}_{i}\left(\phi_{k}(\alpha)\right)-\hat{f}_{i}\left(\phi_{k}\left(\gamma_{i}^{\alpha}\left({\overline{y_{i}^{-}(k), \alpha}}^{-1} d_{M_{k}}-h\right)\right)\right)}{h} \pm \frac{\Psi(\epsilon, \delta ; n, L)}{h}
\end{aligned}
$$

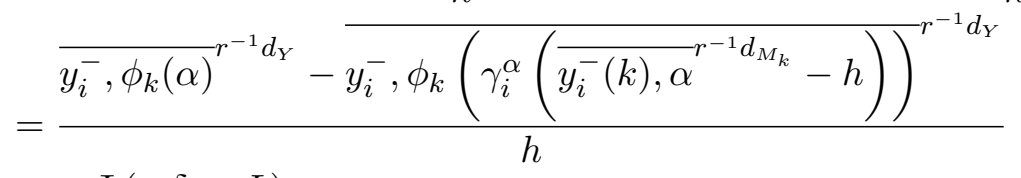

$$
\begin{aligned}
& \pm \frac{\Psi(\epsilon, \delta ; n, L)}{h} \\
& =\frac{{\overline{y_{i}^{-}(k), \alpha}}^{r^{-1} d_{M_{k}}}-\overline{y_{i}^{-}(k), \gamma_{i}^{\alpha}\left({\overline{y_{i}^{-}(k), \alpha}}^{r^{-1} d_{M_{k}}}-h\right)^{r^{-1}} d_{M_{k}}}}{h} \pm \frac{\Psi(\epsilon, \delta ; n, L)}{h} \\
& =1 \pm \frac{\Psi(\epsilon, \delta ; n, L)}{h}
\end{aligned}
$$

for every $k \geq k_{0}$ and every $\alpha \in \hat{B}_{1000}(w(k)) \backslash C_{y_{i}^{-}(k)}$. On the other hand, by an argument similar to the proof of Claim 3.19 , we have

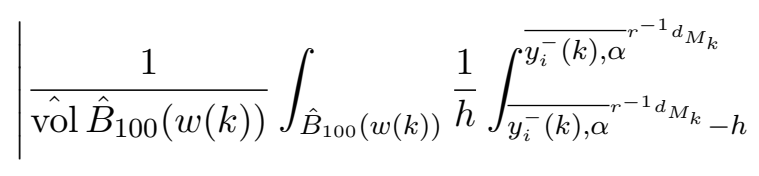




$$
\begin{aligned}
& \left(s-\left(\overline{y_{i}^{-}(k), \alpha} r^{-1} d_{M_{k}}-h\right)\right) \frac{d^{2} \hat{\mathbf{b}}_{i}^{k} \circ \gamma_{i}^{\alpha}}{d s^{2}} d s d \hat{\operatorname{vol}} \mid \\
\leq & C(n) \frac{h}{\hat{\operatorname{vol}} \hat{B}_{1000}(w(k))} \int_{\hat{B}_{1000}(w(k))}\left|\operatorname{Hess}_{\hat{\mathbf{b}}_{i}^{k}}\right|_{r} d \hat{\operatorname{vol}} \leq \Psi(\epsilon, \delta ; n, L) .
\end{aligned}
$$

Since

$$
\begin{aligned}
& \left.\hat{\mathbf{b}}_{i}^{k}(\alpha)=\hat{\mathbf{b}}_{i}^{k}\left(\gamma_{i}^{\alpha}{\overline{y_{i}^{-}(k), \alpha}}^{r^{-1} d_{M_{k}}}-h\right)\right)+\frac{\hat{d} \hat{\mathbf{b}}_{i}^{k}}{\hat{d}_{y_{i}^{-}(k)}}(\alpha) h \\
& -\int{\overline{y_{i}^{-}(k), \alpha}}^{{\overline{y_{i}^{-}(k), \alpha}}^{r^{-1} d_{d_{M_{k}}}}-h}\left(s-\left(\overline{y_{i}^{-}(k), \alpha}{ }^{-1} d_{M_{k}}-h\right)\right) \frac{d^{2} \hat{\mathbf{b}}_{i}^{k} \circ \gamma_{i}^{\alpha}}{d s^{2}} d s
\end{aligned}
$$

for every $\alpha \in \hat{B}_{100}(w(k)) \backslash C_{y_{i}^{-}(k)}$, we have

$$
\frac{1}{\operatorname{vol} \hat{B}_{100}(w(k))} \int_{\hat{B}_{100}(w(k))}\left\langle\hat{d} \hat{\mathbf{b}}_{i}^{k}, \hat{d} \hat{r}_{y_{i}^{-}(k)}\right\rangle_{r} d \hat{\operatorname{vol}}=1 \pm \frac{\Psi(\epsilon, \delta ; n, L)}{h} .
$$

Therefore, we have

$$
\begin{aligned}
& \frac{1}{\operatorname{vol} \hat{B}_{100}(w(k))} \int_{\hat{B}_{100}(x(k))}\left|\hat{d} \hat{f}_{i}^{k}-\hat{d} \hat{r}_{y_{i}^{-}(k)}\right|_{r}^{2} d \hat{\operatorname{vol}} \\
= & \frac{1}{\hat{\operatorname{vol}} \hat{B}_{100}(w(k))} \int_{\hat{B}_{100}(w(k))}\left|\hat{d} \hat{f}_{i}^{k}\right|_{r}^{2} d \hat{\operatorname{vol}} \\
& -\frac{2}{\operatorname{vol} \hat{B}_{100}(w(k))} \int_{\hat{B}_{100}(w(k))}\left\langle\hat{d} \hat{f}_{i}^{k}, \hat{d} \hat{r}_{y_{i}^{-}(k)}\right\rangle_{r} d \hat{\operatorname{vol}}+1 \\
= & 1-2 \frac{1}{\operatorname{vol} \hat{B}_{100}(w(k))} \int_{\hat{B}_{100}(w(k))}\left\langle\hat{d} \hat{\mathbf{b}}_{i}^{k}, \hat{d} \hat{r}_{y_{i}^{-}(k)}\right\rangle_{r} d \hat{\operatorname{vol}}+1 \pm \Psi(\epsilon, \delta ; n, L) \\
= & 2-2\left(1 \pm \frac{\Psi(\epsilon, \delta ; n, L)}{h}\right) \pm \Psi(\epsilon, \delta ; n, L)=\frac{\Psi(\epsilon, \delta ; n, L)}{h} .
\end{aligned}
$$

Therefore, we have Claim 3.26.

Next claim follows from Claim 3.26 and [2, Theorem 9.29] directly:

Claim 3.27. For every sufficiently large $k$, we have

$$
\frac{1}{\operatorname{vol} \hat{B}_{100}(w(k))} \int_{\hat{B}_{1}(w(k))}\left|\left\langle\hat{d} \hat{f}_{i}^{k}, \hat{d} \hat{r}_{z_{j}^{-}(k)}\right\rangle_{r}\right| d \hat{\operatorname{vol}} \leq \Psi(\epsilon, \delta ; n, L)
$$


for every $1 \leq i \leq l$ and every $l+1 \leq j \leq m$. Moreover we have

$$
\frac{1}{\operatorname{vol} \hat{B}_{100}(w(k))} \int_{\hat{B}_{1}(w(k))}\left|\left\langle\hat{d} \hat{f}_{i}^{k}, \hat{d} \hat{f}_{\hat{i}}^{k}\right\rangle_{r}\right| d \hat{\operatorname{vol}} \leq \Psi(\epsilon, \delta ; n, L)
$$

for every $1 \leq i<\hat{i} \leq l$.

For every $i$ with $l+1 \leq i \leq m$, and every sufficiently large $k$, there exists a harmonic function $\hat{\mathbf{b}}_{i}^{k}$ on $\hat{B}_{1000}(w(k))$ such that $\left|\hat{r}_{z_{i}^{-}}-\hat{\mathbf{b}}_{i}^{k}\right|_{L^{\infty}\left(\hat{B}_{1000}(w(k))\right)} \leq$ $\Psi(\epsilon, \delta ; n, L)$ and

$$
\begin{aligned}
& \frac{1}{\hat{\operatorname{vol}} \hat{B}_{1000}(w(k))} \int_{\hat{B}_{1000}(w(k))}\left(\left|\hat{d} \hat{\mathbf{b}}_{i}^{k}-\hat{d} \hat{r}_{z_{i}^{-}(k)}\right|_{r}^{2}+\left|\operatorname{Hess}_{\hat{\mathbf{b}}_{i}^{k}}\right|_{r}^{2}\right) d \hat{\operatorname{vol}} \\
& \quad \leq \Psi(\epsilon, \delta ; n, L) .
\end{aligned}
$$

Let

$$
\begin{aligned}
\hat{F}_{k}= & \sum_{1 \leq i, j \leq m}\left|\left\langle\hat{d} \hat{\mathbf{b}}_{i}^{k}, \hat{d} \hat{\mathbf{b}}_{j}^{k}\right\rangle_{r}-\delta_{i, j}\right|+\sum_{1 \leq i \leq m}\left|\operatorname{Hess}_{\hat{\mathbf{b}}_{i}^{k}}\right|_{r}^{2}+\sum_{i=1}^{l}\left|\hat{d} \hat{\mathbf{b}}_{i}^{k}-\hat{d} \hat{f}_{i}^{k}\right|_{r}^{2} \\
& +\sum_{i=l+1}^{m}\left|\hat{d} \hat{\mathbf{b}}_{i}^{k}-\hat{d} \hat{r}_{z_{i}^{-}}\right|_{r}^{2} .
\end{aligned}
$$

Then, by Lemma 3.1, for every sufficiently large $k$, there exists a compact subset $Z(k)$ of $\hat{\bar{B}}_{1}(w(k))$ such that $\hat{\operatorname{vol}}\left(\hat{B}_{1}(w(k)) \backslash Z(k)\right) / \operatorname{vol} \hat{B}_{1}(w(k)) \leq$ $\Psi(\epsilon, \delta ; n, L)$ and

$$
\frac{1}{\operatorname{vol} \hat{B}_{\hat{s}}(\alpha)} \int_{\hat{B}_{\hat{s}}(\alpha)} \hat{F}_{k} d \hat{\operatorname{vol}} \leq \Psi(\epsilon, \delta ; n, L)
$$

for every $\alpha \in Z(k)$ and every $0<\hat{s}<10$. Thus, by an argument similar to the proof of [6, Theorem 3.3], for every $\alpha \in Z(k)$ and every $0<\hat{s}<1$, there exists a compact subset $P_{s}^{\alpha}$ of $\hat{B}_{\hat{s}}(\alpha)$, a point $p_{\hat{s}}^{\alpha} \in P_{\hat{s}}^{\alpha}$, and a map $q_{\hat{s}}^{\alpha}$ from $\left(\hat{\bar{B}}_{\hat{s}}(\alpha), \alpha\right)$ to $\left(\bar{B}_{\hat{s}}\left(p_{\hat{s}}^{\alpha}\right), p_{\hat{s}}^{\alpha}\right)$ such that the map $Q_{\hat{s}}^{\alpha}=\left(\hat{\mathbf{b}}_{1}^{k}, \ldots, \hat{\mathbf{b}}_{m}^{k}, q_{\hat{s}}^{\alpha}\right)$ from $\hat{\bar{B}}_{\hat{s}}(\alpha)$ to $\hat{\bar{B}}_{\hat{s}+\Psi \hat{s}}\left(\hat{\mathbf{b}}_{1}^{k}(\alpha), \ldots, \hat{\mathbf{b}}_{m}^{k}(\alpha), p_{\hat{s}}^{\alpha}\right)$, is a $\Psi \hat{s}$-Gromov-Hausdorff approximation. By an argument similar to the proof of Claim 3.23, for every $\alpha \in$ $Z(k)$ and every $0<\hat{s}<1$, we have that $\hat{\mathbf{b}}_{i}^{k}=\hat{f}_{i}^{k}+$ constant $\pm \Psi \hat{s}$ on $\hat{B}_{\hat{s}}(\alpha)$ for every $1 \leq i \leq l$, and $\hat{\mathbf{b}}_{i}^{k}=\hat{r}_{z_{i}^{-}(k)}+$ constant $\pm \Psi \hat{s}$ on $\hat{B}_{\hat{s}}(\alpha)$ for every $l+1 \leq i \leq m$. Therefore, the map $\hat{Q}_{\hat{s}}^{\alpha}=\left(\hat{f}_{1}^{k}, \ldots, \hat{f}_{l}^{k}, \hat{r}_{z_{l+1}^{-}(k)}, \ldots, \hat{r}_{z_{m}^{-}(k)}, q_{\hat{s}}^{\alpha}\right)$ from $\hat{\bar{B}}_{\hat{s}}(\alpha)$ to $\hat{\bar{B}}_{\hat{s}+\Psi \hat{s}}\left(\hat{f}_{1}^{k}(\alpha), \ldots, \hat{f}_{l}^{k}(\alpha), \hat{r}_{z_{l+1}^{-}(k)}(\alpha), \ldots, \hat{r}_{z_{m}^{-}(k)}(\alpha), p_{\hat{s}}^{\alpha}\right)$, is a 
$\Psi \hat{s}$-Gromov-Hausdorff approximation. Without loss of generality, we can assume that there exists a compact subset $Z(\infty)$ of $\hat{\bar{B}}_{1}(w)$ such that $Z(k) \rightarrow$ $Z(\infty)$. Let $U=Z(\infty) \cap \bigcap_{1 \leq i \leq l, 1 \leq j \leq k_{i}}\left(\mathcal{D}_{x_{j}^{i}}^{\tau} \backslash\left\{x_{j}^{i}\right\}\right) \cap\left(\mathcal{R}_{m}\right)_{\delta, r}$. By Proposition 2.3 , we have $\hat{v}\left(\hat{B}_{1}(w) \cap U\right) / \hat{v}\left(\hat{B}_{1}(w)\right) \geq 1-\Psi$. Since $\alpha \in\left(\mathcal{R}_{m}\right)_{\tau, \delta}$, we have that the map $T_{\hat{s}}^{\alpha}=\left(\hat{f}_{1}, \ldots, \hat{f}_{l}, \hat{r}_{z_{l+1}^{-}}, \ldots, \hat{r}_{z_{m}^{-}}\right)$from $\hat{B}_{\hat{s}}(\alpha)$ to $\bar{B}_{\hat{s}}\left(T_{\hat{s}}^{\alpha}(\alpha)\right)$, is a $\Psi \hat{s}$-Gromov-Hausdorff approximation for every $\alpha \in U$ and every $0<$ $\hat{s}<1$. Let $\alpha, \beta$ be points in $U \cap \hat{B}_{1 / 2}(w)$ with $\alpha \neq \beta$. Put $\hat{s}=\overline{\alpha, \beta}^{r^{-1} d_{Y}}<1$. Then, we have

$$
\begin{aligned}
& \overline{\left(\hat{f}_{1}(\alpha), \ldots, \hat{f}_{l}(\alpha), \hat{r}_{z_{l+1}^{-}}(\alpha), \ldots, \hat{r}_{z_{m}^{-}}(\alpha)\right),} \\
& \left(\hat{f}_{1}(\beta), \ldots, \hat{f}_{l}(\beta), \hat{r}_{z_{l+1}^{-}}(\beta), \ldots, \hat{r}_{z_{m}^{-}}(\beta)\right) \\
& =\overline{\alpha, \beta}^{r^{-1} d_{Y}} \pm \Psi \hat{s}=(1 \pm \Psi) \overline{\alpha, \beta}^{r^{-1} d_{Y}} \text {. }
\end{aligned}
$$

Therefore, we have the assertion.

Lemma 3.14. Let $(Y, y, v)$ be a Ricci limit space, $l, k, m$ positive integers with $1 \leq l \leq m \leq n, x$ a point in $Y,\left\{h_{i}\right\}_{1 \leq i \leq l}$ a collection of Lipschitz functions on $Y,\left\{x_{i}\right\}_{1 \leq i \leq k}$ of points in $Y$, and $\left\{a_{i}^{j}\right\}_{1 \leq i \leq k, 1 \leq j \leq l}$ of real numbers Let $f_{j}=\sum_{i=1}^{k} a_{i}^{j} r_{x_{i}}$. Assume that the following properties hold:

1. We have

$$
\lim _{r \rightarrow 0} \frac{1}{v\left(B_{r}(x)\right)} \int_{B_{r}(x)}\left|d f_{j}-d h_{j}\right| d v=0
$$

for every $j$.

2. We have

$$
x \in \bigcup_{\tau>0}\left(\bigcap_{\delta>0}\left(\bigcup_{r>0} \operatorname{Leb}\left(\bigcap_{i}\left(\mathcal{D}_{x_{i}}^{\tau} \backslash\left\{x_{i}\right\}\right) \cap\left(\mathcal{R}_{m}\right)_{\delta, r}\right)\right)\right) .
$$

3. The limit

$$
\lim _{r \rightarrow 0} \frac{1}{v\left(B_{r}(x)\right)} \int_{B_{r}(x)}\left\langle d h_{i}, d h_{j}\right\rangle d v \in \mathbf{R}
$$

exists for every $i, j$.

4. We have

$$
\operatorname{det}\left(\lim _{r \rightarrow 0} \frac{1}{v\left(B_{r}(x)\right)} \int_{B_{r}(x)}\left\langle d h_{i}, d h_{j}\right\rangle d v\right)_{i, j} \neq 0
$$


Then, for every $0<\delta<1$, there exists $r_{0}>0$ such that for every $0<s<r_{0}$, there exists compact subset $K_{s}$ of $\bar{B}_{s}(x)$ such that the following properties hold:

1. $v\left(K_{s}\right) / v\left(B_{s}(x)\right) \geq 1-\delta$.

2. For every $\alpha \in K_{s}$ and every sufficiently small $t>0$, there exists a collection $\left\{w_{j}^{t}(\alpha)\right\}_{1 \leq j \leq m-l}$ of points in $Y$, and a compact subset $U_{t}$ of $\bar{B}_{t}(\alpha)$ such that $v\left(U_{t}\right) / v\left(B_{t}(\alpha)\right) \geq 1-\delta$ and that the map $\Phi_{t}=$ $\left(\left(h_{1}, \ldots, h_{l}\right) A, r_{w_{1}^{t}(\alpha)}, \ldots, r_{w_{m-l}^{t}(\alpha)}\right)$ from $U_{t}$ to $\mathbf{R}^{m}$, is a $(1 \pm \delta)-b i$ Lipschitz to the image, where

$$
A=\sqrt{\left(\lim _{r \rightarrow 0} \frac{1}{v\left(B_{r}(x)\right)} \int_{B_{r}(x)}\left\langle d h_{i}, d h_{j}\right\rangle d v\right)_{i, j}^{-1}}
$$

Proof. Define a collection $\left\{g_{i}\right\}_{1 \leq i \leq l}$ of Lipschitz functions $g_{i}$ on $Y$ by $\left(g_{1}, \ldots, g_{l}\right)=\left(h_{1}, \ldots, h_{l}\right) A$. By the definition, we have

$$
\lim _{r \rightarrow 0} \frac{1}{v\left(B_{r}(x)\right)} \int_{B_{r}(x)}\left\langle g_{i}, g_{j}\right\rangle d v=\delta_{i, j} .
$$

By the assumption and Corollary 3.1, we have

$$
\lim _{r \rightarrow 0} \frac{1}{v\left(B_{r}(x)\right)} \int_{B_{r}(x)}\left|\left\langle g_{i}, g_{j}\right\rangle-\delta_{i, j}\right| d v=0 .
$$

Put $\quad\left(F_{1}, \ldots, F_{l}\right)=\left(\sum_{i=1}^{k} b_{i}^{1} r_{x_{i}}, \ldots, \sum_{i=1}^{k} b_{i}^{l} r_{x_{i}}\right)=\left(\sum_{i=1}^{k} a_{i}^{1} r_{x_{i}}, \ldots\right.$, $\left.\sum_{i=1}^{k} a_{i}^{l} r_{x_{i}}\right) A$. Let $L \geq 1$ satisfying $|A|+\sum_{i, j}\left(b_{i}^{j}\right)^{2} \leq L$. Fix $0<\delta<1$. By Lemma 3.13, we have the following claim:

Claim 3.28. There exists $r_{1}>0$ such that for every $0<s \leq r_{1}$, there exist a compact subset $K_{s}$ of $\bar{B}_{s}(x)$ such that the following properties hold:

1. $v\left(K_{s}\right) / v\left(B_{s}(x)\right) \geq 1-\delta$.

2. For every $\alpha \in K_{s}$ and every sufficiently small $t>0$, there exist a collection of points $\left\{w_{j}^{t}(\alpha)\right\}_{1 \leq j \leq m-l}$ in $Y$, and a compact subset $E_{t}$ of $\bar{B}_{t}(\alpha)$ such that $v\left(E_{t}\right) / v\left(B_{t}(\alpha)\right) \geq 1-\delta$ and that the map $\Phi_{t}^{\alpha}=$ $\left(F_{1}, \ldots, F_{l}, r_{w_{1}^{t}(\alpha)}, \ldots, r_{w_{m-l}^{t}(\alpha)}\right)$ from $E_{t}$ to $\mathbf{R}^{m}$, is $(1 \pm \delta)$-bi-Lipschitz to the image. 
On the other hand, there exists $r_{0}>0$ such that

$$
\frac{1}{v\left(B_{s}(x)\right)} \int_{B_{s}(x)} \sum_{j}\left|d F_{j}-d g_{j}\right| d v \leq \delta
$$

for every $0<s<r_{0}$. Thus, by Lemma 3.1, for every $0<s<r_{0} / 100$, there exists a compact subset $X_{s}$ of $\bar{B}_{s}(x)$ such that $v\left(X_{s}\right) / v\left(\bar{B}_{s}(x)\right) \geq 1-\Psi(\delta ; n)$ and

$$
\frac{1}{v\left(B_{5 \hat{s}}(\alpha)\right)} \int_{B_{5 \hat{s}}(\alpha)} \sum_{j}\left|d F_{j}-d g_{j}\right| d v \leq \Psi(\delta ; n)
$$

for every $\alpha \in X_{s}$ and every $0<\hat{s} \leq s$. Put $V_{s}=K_{s} \cap X_{s}$ for every $0<$ $s<\min \left\{r_{0}, r_{1}\right\} / 1000$. Then we have $v\left(V_{s}\right) / v\left(B_{s}(x)\right) \geq 1-\Psi(\delta ; n)$. Fix $0<$ $s<\min \left\{r_{0}, r_{1}\right\} / 1000$ and $\alpha \in V_{s}$. By an argument similar to the proof of Claim 3.23, for every sufficiently small $t>0$, we have $F_{j}=f_{j}+$ constant \pm $\Psi(\delta ; n) t$ on $\bar{B}_{t}(\alpha)$. Fix such $t>0$ and put $U_{t}=B_{t / 2}(\alpha) \cap E_{t}$. Then, we have $v\left(U_{t}\right) / v\left(B_{t / 2}(\alpha)\right) \geq 1-\Psi(\delta ; n)$. Let $p_{1}, p_{2}$ be points in $U_{t}$ with $p_{1} \neq p_{2}$. Put $\hat{t}=\overline{p_{1}, p_{2}}>0$. Then, we have

$$
\begin{aligned}
& \left(f_{1}\left(p_{1}\right), \ldots, f_{l}\left(p_{1}\right), r_{w_{1}^{t}(\alpha)}, \ldots, r_{w_{m-l}^{t}(\alpha)}\left(p_{1}\right)\right), \\
& \left(f_{1}\left(p_{2}\right), \ldots, f_{l}\left(p_{2}\right), r_{w_{1}^{t}(\alpha)}\left(p_{2}\right), \ldots, r_{w_{m-l}^{t}(\alpha)}\left(p_{2}\right)\right)
\end{aligned}
$$

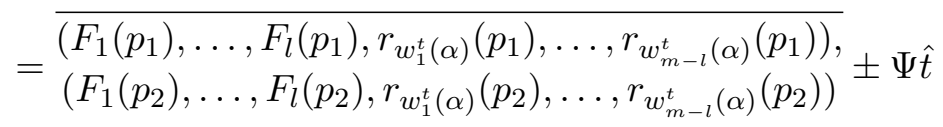

$$
\begin{aligned}
& =(1 \pm \delta) \overline{p_{1}, p_{2}} \pm \Psi \hat{t}=(1 \pm \Psi) \overline{p_{1}, p_{2}} \text {. }
\end{aligned}
$$

Therefore, we have the assertion.

Lemma 3.15. Let $(Y, y, v)$ be a Ricci limit space, $l$ a positive integer, $\left\{f_{i}\right\}_{1 \leq i \leq l}$ a collection of Lipschitz functions on $Y, f$ a Lipschitz function on $Y$, and $A$ a Borel subset of $Y$. Assume that $\operatorname{span}\left\{d f_{1}(x), \ldots, d f_{l}(x)\right\}=T_{x}^{*} Y$ for a.e. $x \in A$. Then, for a.e. $x \in A$, there exists a collection of real numbers $\left\{b_{i}(x)\right\}_{1 \leq i \leq l}$ such that

$$
\lim _{r \rightarrow 0} \frac{1}{v\left(B_{r}(x)\right)} \int_{B_{r}(x)}\left|d f-\sum_{i=1}^{l} b_{i}(x) d f_{i}\right|^{2} d v=0
$$

Proof. Without loss of generality, we can assume that $\left\{d f_{i}(x)\right\}_{i}$ is a basis of $T_{x}^{*} Y$ for every $x \in A$. Put

$$
\left(b_{1}(x), \ldots, b_{l}(x)\right)=\left(\left\langle d f, d f_{1}\right\rangle(x), \ldots,\left\langle d f, d f_{l}\right\rangle(x)\right){\sqrt{\left(\left\langle d f_{i}, d f_{j}\right\rangle(x)\right)_{i, j}}}^{-1}
$$


for every $x \in A$. Then, by Lebesgue's differentiation theorem, for a.e. $x \in A$, we have

$$
\begin{aligned}
& \lim _{r \rightarrow 0} \frac{1}{v\left(B_{r}(x)\right)} \int_{B_{r}(x)}|d f|^{2} d v=|d f|^{2}(x), \\
& \lim _{r \rightarrow 0} \frac{1}{v\left(B_{r}(x)\right)} \int_{B_{r}(x)}\left\langle d f, d f_{i}\right\rangle d v=\left\langle d f, d f_{i}\right\rangle(x)
\end{aligned}
$$

and

$$
\lim _{r \rightarrow 0} \frac{1}{v\left(B_{r}(x)\right)} \int_{B_{r}(x)}\left\langle d f_{i}, d f_{j}\right\rangle d v=\left\langle d f_{i}, d f_{j}\right\rangle(x)
$$

for every $i, j$. Then, since it is easy to check that

$$
\begin{aligned}
\lim _{r \rightarrow 0} \frac{1}{v\left(B_{r}(x)\right)} \int_{B_{r}(x)}|d f|^{2} d v & =\lim _{r \rightarrow 0} \frac{1}{v\left(B_{r}(x)\right)} \int_{B_{r}(x)}\left\langle d f, \sum_{i=1}^{l} b_{i}(x) d f_{i}\right\rangle d v \\
& =\lim _{r \rightarrow 0} \frac{1}{v\left(B_{r}(x)\right)} \int_{B_{r}(x)}\left|\sum_{i=1}^{l} b_{i}(a) d f_{i}\right|^{2} d v \\
& =\left|\sum_{i=1}^{l} b_{i}(x) d f_{i}(x)\right|^{2}
\end{aligned}
$$

for a.e. $x \in A$, we have

$$
\begin{aligned}
& \lim _{r \rightarrow 0} \frac{1}{v\left(B_{r}(x)\right)} \int_{B_{r}(x)}\left|d f-\sum_{i=1}^{l} b_{i}(x) d f_{i}\right|^{2} d v \\
& =\lim _{r \rightarrow 0} \frac{1}{v\left(B_{r}(x)\right)} \int_{B_{r}(x)}|d f|^{2} d v-2 \lim _{r \rightarrow 0} \frac{1}{v\left(B_{r}(x)\right)} \int_{B_{r}(x)}\left\langle d f, \sum_{i=1}^{l} b_{i}(x) d f_{i}\right\rangle d v \\
& \quad+\lim _{r \rightarrow 0} \frac{1}{v\left(B_{r}(x)\right)} \int_{B_{r}(x)}\left|\sum_{i=1}^{l} b_{i}(a) d f_{i}\right|^{2} d v=0
\end{aligned}
$$

for a.e. $x \in A$.

Theorem 3.4 (Rectifiability associated with Lipschitz functions). Let $(Y, y, v)$ be a Ricci limit space, $l$ a positive integer, $\left\{f_{i}\right\}_{1 \leq i \leq l}$ a collection of Lipschitz functions on $Y, A$ a Borel subset of $Y$. Assume that $\left\{d f_{i}(x)\right\}_{1 \leq i \leq l}$ are linearly independent in $T_{x}^{*} Y$ for a.e. $x \in A$. Then, there exist $0<\alpha(n)<$ 1 , collections of compact subsets $\left\{C_{k, i}\right\}_{l \leq k \leq n, i \in N}$ of $A$, of points $\left\{x_{k, i}\right\}_{k, i}$ in 
A, and of points $\left\{x_{k, i}^{s}\right\}_{k, i, 1 \leq s \leq k-l}$ in $Y$ such that the following properties hold:

1. $C_{k, i} \subset \mathcal{R}_{k, \alpha(n)} \cap \bigcap_{j=1}^{k-l}\left(A \backslash\left(C_{x_{k, i}^{j}} \cup\left\{x_{k, i}^{j}\right\}\right)\right) \quad$ and $\quad v\left(A \backslash \bigcup_{l \leq k \leq n, i \in N}\right.$ $\left.C_{k, i}\right)=0$ for evrey $k$.

2. For every $l \leq k \leq n$, every $x \in \bigcup_{i \in \mathbf{N}} C_{k, i}$ and every $0<\delta<1$, there exists $C_{k, i}$ such that $x \in C_{k, i}$ and that the map $\phi_{k, i}=\left(\left(f_{1}, \ldots, f_{l}\right)\right.$ \left.${\sqrt{\left(\left\langle d f_{i}, d f_{j}\right\rangle\left(x_{k, i}\right)\right)_{i, j}}}^{-1}, r_{x_{k, i}^{1}}, \ldots, r_{x_{k, i}^{k-l}}\right)$ from $C_{k, i}$ to $\mathbf{R}^{k}$, is $(1 \pm \delta)-b i$ Lipschitz to the image.

3. The limit measure $v$ and the $k$-dimensional Hausdorff measure $H^{k}$ are mutually absolutely continuous on $C_{k, i}$. Moreover, $v$ is Ahlfors $k$-regular at every $x \in C_{k, i}$.

Proof. Let $\left\{C_{k, i}^{y}\right\}_{k, i}$ be a collection of Borel subset of $Y$, and $\left\{x_{k, i}^{\hat{l}}\right\}_{k, i}$ of points in $Y$ as in Theorem 3.1, where $x_{k, i}^{1}=y$. By Lemma 3.5, without loss of generality, we can assume that $C_{k, i}$ is bounded for every $i, k$. By the construction of $T^{*} Y$, we have $\operatorname{span}\left\{d r_{x_{k, i}^{1}}(x), \ldots, d r_{x_{k, i}^{k}}(x)\right\}=T_{x}^{*} Y$ for a.e.

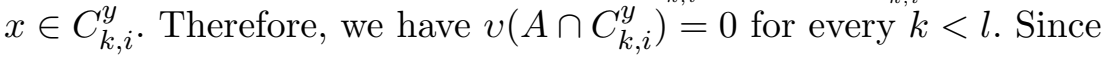

$$
v\left(\mathcal{R}_{k} \backslash \bigcup_{\tau>0}\left(\bigcap_{\delta>0}\left(\bigcup_{r>0} \operatorname{Leb}\left(\bigcap_{i, j}\left(\mathcal{D}_{x_{i}^{j}}^{\tau} \backslash\left\{x_{i}^{j}\right\}\right) \cap\left(\mathcal{R}_{k}\right)_{\delta, r}\right)\right)\right)\right)=0
$$

the following claim follows from Lemmas 3.14 and 3.15, directly:

Claim 3.29. For every $k \geq l$ and every $i \in \mathbf{N}$, there exists a Borel subset $A_{k, i}$ of $A \cap C_{k, i}$ such that the following properties hold:

1. $v\left(A \cap C_{k, i} \backslash A_{k, i}\right)=0$.

2. For every $x \in A_{k, i}$ and every $0<\delta<1$, there exists $r_{x}^{\delta}>0$ such that for every $0<s<r_{x}^{\delta}$, there exists a compact subset $K(x, \delta, s)$ of $\bar{B}_{s}(x)$ such that the following properties hold:

(a) $v(K(x, \delta, s)) / v\left(B_{s}(x)\right) \geq 1-\delta$.

(b) For every $\alpha \in K(x, \delta, s)$ and every sufficiently small $t>0$, there exist a collection of points $\{w(i, x, \delta, s, \alpha, t)\}_{1 \leq i \leq k-l}$ in $Y$, and a compact subset $U(x, \delta, s, \alpha, t)$ of $\bar{B}_{t}(\alpha)$ such that the map $\Phi^{x, \delta, s, \alpha, t}=$ $\left(\left(f_{1}, \ldots, f_{l}\right) A(x), r_{w(1, x, \delta, s, \alpha, t)}, \ldots, r_{w(k-l, x, \delta, s, \alpha, t)}\right)$ from $U(x, \delta, s, \alpha, t)$ 
to $\mathbf{R}^{k}$, is $(1 \pm \delta)$-bi-Lipschitz to the image, where

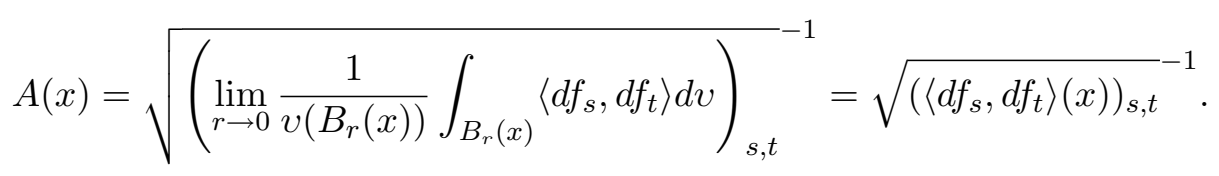

Put $\hat{A}_{k, i}=\operatorname{Leb}\left(A_{k, i}\right)$. For every $N \in \mathbf{N}$ and every $x \in \hat{A}_{k, i}$, let $s_{x}^{N}$ be a positive number satisfying that $0<s_{x}^{N}<\min \left\{r_{x}^{1 / N}, N^{-1}\right\}$ and $v\left(B_{s_{x}^{N}}(x) \cap\right.$ $\left.A_{k, i}\right) / v\left(B_{s_{x}^{N}}(x)\right) \geq 1-N^{-1}$. Let $K\left(x, N^{-1}, s_{x}^{N}\right)$ be a compact subset as in Claim 3.29. Put $\hat{K}\left(x, N^{-1}, s_{x}^{N}\right)=K\left(x, N^{-1}, s_{x}^{N}\right) \cap \hat{A}_{k, i}$. Then, we have $v\left(B_{s_{x}^{N}}(x) \cap \hat{K}\left(x, N^{-1}, s_{x}^{N}\right)\right) / v\left(B_{s_{x}^{N}}(x)\right) \geq 1-100 N^{-1}$. For every $\alpha \in \hat{K}(x$, $\left.N^{-1}, s_{x}^{N}\right)$, there exists $0<t=t(\alpha)<N^{-1}$ such that $v\left(B_{\hat{t}}(\alpha) \cap A_{k, i}\right) / v\left(B_{\hat{t}}(\alpha)\right)$ $\geq 1-N^{-1}$ for every $0<\hat{t}<t$. Take $w\left(i, x, N^{-1}, s_{x}^{N}, \alpha, \hat{t}\right)$ and $U\left(x, N^{-1}\right.$, $\left.s_{x}^{N}, \alpha, \hat{t}\right)$ as in Claim 3.29. Put $\hat{U}\left(x, N^{-1}, s_{x}^{N}, \alpha, \hat{t}\right)=U\left(x, N^{-1}, s_{x}^{N}, \alpha, \hat{t}\right) \cap$ $\hat{A}_{k, i}$. Then, we have $v\left(B_{\hat{t}}(\alpha) \cap \hat{U}\left(x, N^{-1}, s_{x}^{N}, \alpha, \hat{t}\right)\right) / v\left(B_{\hat{t}}(\alpha)\right) \geq 1-1000 N^{-1}$.

By Lemma 2.2, it is not difficult to check that the following claim:

Claim 3.30. There exist $x_{j}^{N} \in \hat{A}_{k, i}, \alpha_{j}^{N} \in \hat{K}\left(x_{j}^{N}, N^{-1}, s_{x_{j}^{N}}^{N}\right)$ and $0<t_{j}^{N}<$ $t\left(\alpha_{j}^{N}\right)$ such that

$$
v\left(A_{k, i} \backslash \bigcup_{j \in \mathbf{N}} \hat{U}\left(x_{j}^{N}, N^{-1}, s_{x_{j}^{N}}^{N}, \alpha_{j}^{N}, t_{j}^{N}\right)\right) \leq \Psi\left(N^{-1} ; n\right) v\left(B_{10}\left(A_{k, i}\right)\right) .
$$

Put $\hat{U}(j, N)=\hat{U}\left(x_{j}^{N}, N^{-1}, s_{x_{j}^{N}}^{N}, \alpha_{j}^{N}, t\left(\alpha_{j}^{N}\right)\right), w(i, j, N)=w\left(i, x_{j}^{N}, N^{-1}\right.$, $\left.s_{x_{j}^{N}}^{N}, \alpha_{j}^{N}, t\left(\alpha_{j}^{N}\right)\right), U(j)=\bigcap_{N_{0} \in \mathbf{N}}\left(\bigcup_{N_{1} \geq N_{0}} \hat{U}\left(j, N_{1}\right)\right)$ and $U(j, N)=\hat{U}(j, N) \cap$ $U(j)$. Then we have $v\left(A_{k, i} \backslash \bigcup_{j \in \mathbf{N}} U(j)\right)=0$ and $\bigcup_{N \in \mathbf{N}} U(j, N)=U(j)$. Fix $j, w \in \bigcup_{N \in \mathbf{N}} U(j, N)$ and $0<\delta<1$. There exists $N_{0}$ such that $w \in$ $U\left(j, N_{0}\right)$. Let $N_{1} \in \mathbf{N}$ with $N_{1}^{-1}<<\delta$. Since $w \in \bigcup_{N_{2} \geq N_{1}} \hat{U}\left(j, N_{2}\right)$, there exists $N_{2} \geq N_{1}$ such that $w \in \hat{U}\left(j, N_{2}\right)$. Especially, we have $w \in U\left(j, N_{2}\right)$. Thus, the map $G_{j, N_{2}}=\left(\left(f_{1}, \ldots, f_{l}\right) A\left(x_{j}^{N_{2}}\right), r_{w\left(1, j, N_{2}\right)}, \ldots, r_{w\left(k-l, j, N_{2}\right)}\right)$ from $U\left(j, N_{2}\right)$ to $\mathbf{R}^{k}$, is $\left(1 \pm N_{2}^{-1}\right)$-bi-Lipschitz to the image. Especially, $G_{j, N_{2}}$ is $(1 \pm \delta)$-bi-Lipschitz to the image. Therefore, we have the assertion.

Remark 3.2. The radial rectifiability theorem, Theorem 3.1, corresponds to Theorem 3.4 for the case: $l=1, f_{1}=r_{x}, A=Y$.

We will end this subsection by giving two corollaries of Theorem 3.4. For a metric space $X$, define a distance on $\mathbf{R}_{\geq 0} \times X /(\{0\} \times X)$ by $\overline{\left(t_{1}, x_{1}\right),\left(t_{2}, x_{2}\right)}=$ 
$\sqrt{t_{1}^{2}+t_{2}^{2}-2 t_{1} t_{2} \cos \min \left\{\overline{x_{1}, x_{2}}, \pi\right\}}$. Denote this metric space by $C(X)$, and put $p=[(0, x)] \in C(X)$. The next corollary is used in [24], essentially.

Corollary 3.2. Let $X$ be a compact geodesic space and $l$ a non-negative integer. Assume that $l \leq n, \operatorname{dim}_{H} X=n-l-1$ and that $\left(\mathbf{R}^{l} \times C(X),\left(0_{l}, p\right)\right)$ is a Ricci limit space. Then, $X$ is $H^{n-l-1}$-rectifiable.

Proof. Define a collection of 1-Lipschitz functions $\{g\} \cup\left\{\pi_{j}\right\}_{1 \leq j \leq l}$ on $\mathbf{R}^{k} \times$ $C(X)$ by $\pi_{j}\left(t_{1}, \ldots, t_{l}, w\right)=t_{j}$ and $g\left(t_{1}, \ldots, t_{l}, w\right)=\overline{p, w}$. By Theorem 3.3 and $\left[4\right.$, Theorem 5.9], we have $\left\langle d \pi_{i}, d \pi_{j}\right\rangle(\alpha)=\delta_{i, j},\left\langle d \pi_{i}, d g\right\rangle(\alpha)=0,|d g|(\alpha)=$ 1 for a.e. $\alpha \in \mathbf{R}^{l} \times C(X)$ with respect to the $n$-dimensional Hausdorff measure $H^{n}$. Therefore, by applying Theorem 3.4 for a collection of Lipschitz functions $\left\{\pi_{j}\right\}_{1 \leq j \leq l} \cup\{g\}$ and $A=\mathbf{R}^{l} \times C(X)$, there exists a collection of Borel subsets $\left\{C_{k, i}\right\}_{i, l+1 \leq k \leq n}$ as in Theorem 3.4. Since the product measure $H^{l} \times H^{n-l}$ on $\mathbf{R}^{l} \times C(X)$ is equal to $H^{n}$ (see the appendix in [24]), by Fubini's theorem, we have

$$
\begin{aligned}
0 & =H^{n}\left(\left(\mathbf{R}^{l} \times C(X)\right) \backslash \bigcup_{k, i} C_{k, i}\right) \\
& =\int_{\mathbf{R}^{l}} H^{n-l}\left(\left(\left\{t_{1}, \ldots, t_{l}\right\} \times C(X)\right) \backslash \bigcup_{k, i} C_{k, i}\right) d H^{l} .
\end{aligned}
$$

Especially, there exists $\left(t_{1}, \ldots, t_{l}\right) \in \mathbf{R}^{l}$ such that $H^{n-l}\left(\left(\left\{t_{1}, \ldots, t_{l}\right\} \times C(X)\right)\right.$ $\left.\backslash \bigcup_{k, i} C_{k, i}\right)=0$. Put $\hat{C}_{k, i}=\left(\left\{t_{1}, \ldots, t_{l}\right\} \times C(X)\right) \cap C_{k, i}$ and regard it as a subset of $C(X)$, canonically. Now, we remark that

$$
\int_{C(X)} f d H^{n-l}=\int_{0}^{\infty} \int_{\partial B_{t}(p)} f d H^{n-l-1} d t
$$

holds for every $f \in L^{1}(C(X))$ (this is the co-area formula for the distance function from the pole in $C(X)$. See for instance the appendix in [24]). Thus, especially, we have $H^{n-l-1}\left(\partial B_{t}(p) \cap C(X) \backslash \bigcup_{k, i} \hat{C}_{k, i}\right)=0$ for a.e. $t>0$. Then it is not difficult to check the assertion.

Remark 3.3. With the same notation as in Corollary 3.2, we have $0<$ $H^{n-l-1}\left(B_{r}(x)\right)<\infty$ for every $x \in X$ and every $r>0$. It follows from [4, Theorem 5.9],[6, Theorem 4.6] and the above co-area formula for the distance function from the pole on $C(X)$. We skipped the proof because it is not difficult to check it. 
Similarly, we have the following:

Corollary 3.3. Let $(X, x)$ be a pointed proper geodesic space, $l$ a nonnegative integer. Assume that $l \leq n, \operatorname{dim}_{H} X=n-l$ and that $\left(\mathbf{R}^{l} \times X,\left(0_{l}, x\right)\right)$ is a Ricci limit space. Then, $X$ is $H^{n-l}$-rectifiable.

\section{Convergence of $L^{\infty}$-functions and of Lipschitz functions}

In this section, we will give two-notions of convergence of a sequence of $L^{\infty}$-functions with respect to the measured Gromov-Hausdorff topology. By using these notions, we will give the definition of a convergence of the differentials of Lipschitz functions (see Definition 4.4). Moreover, by combining with several results given in Section 3, we will discuss convergence of harmonic functions. In [26-30], we can also find related important, interesting results to this section. For harmonic functions, see also $[9,11,24,31-33$, $36,37]$. Throughout the following Subsections 4.1 and 4.2 , we shall fix the following:

1. Let $\left\{\left(Z_{i}, z_{i}\right)\right\}_{1 \leq i \leq \infty}$ be a sequence of pointed proper geodesic spaces, $x_{i} \in Z_{i}$.

2. Let $v_{i}$ be a Radon measure on $Z_{i}$ for every $1 \leq i \leq \infty$.

3. $v_{i}\left(B_{1}\left(z_{i}\right)\right)=1$ holds for every $i$.

4. For every $R \geq 1$, there exists $\kappa=\kappa(R) \geq 1$ such that $v_{i}\left(B_{2 s}(z)\right) \leq$ $2^{\kappa} v_{i}\left(B_{s}(z)\right)$ for every $1 \leq i \leq \infty$, every $z \in Z_{i}$ and every $0<s \leq R$.

5. $\left(Z_{i}, x_{i}, z_{i}, v_{i}\right) \stackrel{\left(\phi_{i}, R_{i}, \epsilon_{i}\right)}{\longrightarrow}\left(Z_{\infty}, x_{\infty}, z_{\infty}, v_{\infty}\right)$.

\subsection{Pointwise strong convergence of $L^{\infty}$-functions}

Our aims in this subsection are to give the following notion and several fundamental properties of it:

Definition 4.1 (Pointwise strong convergence of $L^{\infty}$-functions). Let $R$ be a positive number, $w_{\infty}$ a point in $B_{R}\left(x_{\infty}\right)$ and $\left\{f_{i}\right\}_{1 \leq i \leq \infty}$ a sequence of $L^{\infty}$ functions $f_{i}$ on $B_{R}\left(x_{i}\right)$ with $\sup _{i}\left|f_{i}\right|_{L^{\infty}\left(B_{R}\left(x_{i}\right)\right)}<\infty$. We say that $f_{i}$ converges strongly to $f_{\infty}$ at $w_{\infty}$ if for every $\epsilon>0$, there exists $r>0$ such that 


$$
\limsup _{i \rightarrow \infty} \frac{1}{v_{i}\left(B_{t}\left(w_{i}\right)\right)} \int_{B_{t}\left(w_{i}\right)}\left|f_{i}-\frac{1}{v_{\infty}\left(B_{t}\left(w_{\infty}\right)\right)} \int_{B_{t}\left(w_{\infty}\right)} f_{\infty} d v_{\infty}\right| d v_{i} \leq \epsilon
$$

and

$$
\limsup _{i \rightarrow \infty} \frac{1}{v_{\infty}\left(B_{t}\left(w_{\infty}\right)\right)} \int_{B_{t}\left(w_{\infty}\right)}\left|f_{\infty}-\frac{1}{v_{i}\left(B_{t}\left(w_{i}\right)\right)} \int_{B_{t}\left(w_{i}\right)} f_{i} d v_{i}\right| d v_{\infty} \leq \epsilon
$$

for every $0<t<r$ and every $w_{i} \rightarrow w_{\infty}$.

Example 4.1. Fix $f \in C^{0}\left(B_{R}\left(x_{\infty}\right)\right)$ and put $f_{i}=f \circ \phi_{i}$. Then, it is easy to check that $f_{i}$ converges strongly to $f_{\infty}$ at every $w \in B_{R}\left(x_{\infty}\right)$.

We shall give a fundamental result about this convergence without the proof because it is not difficult to check it:

Proposition 4.1. Let $k$ be a positive integer, $R$ a positive number, $\left\{f_{i}^{l}\right\}_{1 \leq l \leq k}$ a collection of $L^{\infty}$-functions on $B_{R}\left(x_{i}\right)$ for every $1 \leq i \leq \infty$ with $\sup _{i, l}\left|f_{i}^{\bar{l}}\right|_{L^{\infty}\left(B_{R}\left(x_{i}\right)\right)}<\infty, w_{\infty}$ a point in $B_{R}\left(x_{\infty}\right)$ and $\left\{F_{i}\right\}_{1 \leq i \leq \infty}$ a sequence of continuous functions on $\mathbf{R}^{k}$. Assume that $f_{i}^{l}$ converges strongly to $f_{\infty}^{l}$ at $w_{\infty}$ for every $l$, and that $F_{i}$ converges to $F_{\infty}$ with respect to the compact uniformly topology. Then, $F_{i}\left(f_{i}^{1}, \ldots, f_{i}^{k}\right)$ converges strongly to $F_{\infty}\left(f_{\infty}^{1}, \ldots, f_{\infty}^{k}\right)$ at $w_{\infty}$.

Remark 4.1. Let $k$ be a positive integer, $\left\{f_{i}^{l}\right\}_{1 \leq l \leq k}$ a collection of $L^{\infty}$ functions $f_{i}^{l}$ on $B_{R}\left(x_{i}\right)$ for every $1 \leq i \leq \infty, w_{\infty}$ a point in $B_{R}\left(x_{\infty}\right)$, and $\left\{F_{i}\right\}_{1 \leq i \leq \infty}$ a sequence of locally $L^{\infty}$-functions on $\mathbf{R}^{k}$. Assume that the following properties hold:

1. $\sup _{i, l}\left|f_{i}^{l}\right|_{L^{\infty}\left(B_{R}\left(x_{i}\right)\right)}<\infty$.

2. $f_{i}^{l}$ converges strongly to $f_{\infty}^{l}$ at $w_{\infty}$ for every $l$.

3. The limits

$$
a^{l}=\lim _{r \rightarrow 0} \frac{1}{v_{\infty}\left(B_{r}\left(w_{\infty}\right)\right)} \int_{B_{r}\left(w_{\infty}\right)} f_{\infty}^{l} d v_{\infty} \in \mathbf{R}
$$

exist for every $l$.

4. There exists an open neighborhood $U$ at $\left(a^{1}, \ldots, a^{k}\right) \in \mathbf{R}^{k}$ such that $F_{i}$ is continuous on $U$ for every $1 \leq i \leq \infty$, and that $F_{i}$ converges to $F_{\infty}$ on $U$ uniformly. 
Then, we also have that $F_{i}\left(f_{i}^{1}, \ldots, f_{i}^{k}\right)$ converges strongly to $F_{\infty}\left(f_{\infty}^{1}, \ldots, f_{\infty}^{k}\right)$ at $w_{\infty}$.

The following proposition is the main result in this subsection:

Proposition 4.2. Let $\left\{\left(M_{i}, m_{i}\right)\right\}_{i}$ be a sequence of pointed $n$-dimensional complete Riemannian manifolds with $\operatorname{Ric}_{M_{i}} \geq-(n-1),(Y, y, v)$ a Ricci limit space of $\left\{\left(M_{i}, m_{i}, \underline{\mathrm{vol}}\right)\right\}_{i}, R$ a positive number, $x_{\infty}, z_{\infty}$ points in $Y$, $x_{i}, z_{i}$ points in $M_{i}$ for every $i<\infty, f_{i}$ a $C^{2}$-function on $B_{R}\left(x_{i}\right)$ for every $i<\infty$, and $f_{\infty}$ a Lipschitz function on $B_{R}\left(x_{\infty}\right)$. Assume that $\sup _{i} \operatorname{Lip} f_{i}<$ $\infty,\left(M_{i}, m_{i}, x_{i}, z_{i}, f_{i}, \underline{\mathrm{vol}}\right) \stackrel{\left(\phi_{i}, R_{i}, \epsilon_{i}\right)}{\longrightarrow}\left(Y, y, x_{\infty}, z_{\infty}, f_{\infty}, v\right)$ and

$$
\sup _{i} \int_{B_{R}\left(x_{i}\right)}\left|\operatorname{Hess}_{f_{i}}\right| d \underline{\mathrm{vol}}<\infty .
$$

Then, $\left\langle d r_{z_{i}}, d f_{i}\right\rangle$ converges strongly to $\left\langle d r_{z_{\infty}}, d f_{\infty}\right\rangle$ at a.e. $w_{\infty} \in B_{R}\left(x_{\infty}\right)$.

Proof. Fix $\epsilon>0$ and let $L \geq 1$ with

$$
\sup _{i}\left(\frac{1}{\underline{\operatorname{vol}} B_{R}\left(x_{i}\right)} \int_{B_{R}\left(x_{i}\right)}\left|\operatorname{Hess}_{f_{i}}\right| d \underline{\mathrm{vol}}+\operatorname{Lip} f_{i}\right) \leq L
$$

By Theorem 3.3, there exist $0<\eta<<\epsilon$ and a Borel subset $X(\epsilon)$ of $B_{R}\left(x_{\infty}\right) \cap$ $\mathcal{D}_{z}^{\eta} \backslash B_{\eta}\left(z_{\infty}\right)$ such that $v\left(B_{R}\left(x_{\infty}\right) \backslash X(\epsilon)\right) / v\left(B_{R}\left(x_{\infty}\right)\right) \leq \epsilon$ and

$$
\left|\frac{f_{\infty} \circ \gamma(\overline{z, \alpha}+h)-f_{\infty}(\alpha)}{h}-\left\langle d r_{z_{\infty}}, d f_{\infty}\right\rangle(\alpha)\right| \leq \epsilon
$$

for every $\alpha \in X(\epsilon)$, every real number $h$ with $0<|h|<\eta$, and every isometric embedding $\gamma$ from $\left[0, \overline{z_{\infty}, \alpha}+\eta\right]$ to $Y$ with $\gamma(0)=z_{\infty}, \gamma\left(\overline{z_{\infty}, \alpha}\right)=\alpha$. On the other hand, by Lebesgue's differentiation theorem, there exists a Borel subset $\hat{X}(\epsilon)$ of $X(\epsilon)$ such that $v(X(\epsilon) \backslash \hat{X}(\epsilon))=0$ and that for every $\alpha \in \hat{X}(\epsilon)$, there exists $r(\alpha)>0$ such that

$$
\frac{1}{v\left(B_{t}(\alpha)\right)} \int_{B_{t}(\alpha)}\left|\left\langle d r_{z_{\infty}}, d f_{\infty}\right\rangle-\left\langle d r_{z_{\infty}}, d f_{\infty}\right\rangle(\alpha)\right| d v<\epsilon
$$

for every $0<t<r(\alpha)$. Put $l=\eta^{-1 / 4}$. By an argument similar to the proof of Proposition 3.1, for every $1 \leq i<\infty$, there exists a compact subset $K_{i}$ of 
$B_{R-\epsilon}\left(x_{i}\right)$ such that

$\frac{\underline{\operatorname{vol}}\left(B_{R-\epsilon}\left(x_{i}\right) \backslash K_{i}\right)}{\underline{\operatorname{vol}} B_{R-\epsilon}\left(x_{i}\right)} \leq \Psi\left(l^{-1} ; n, R, L\right)$ and $\frac{1}{\underline{\operatorname{vol} B_{t}(w)}} \int_{B_{t}(w)}\left|\operatorname{Hess}_{f_{i}}\right| d \underline{\operatorname{vol}} \leq l$

for every $w \in K_{i}$ and every $0<t<\epsilon / 100$. Without loss of generality, we can assume that there exists a compact subset $K_{\infty}$ of $\bar{B}_{R}\left(x_{\infty}\right)$ such that $K_{i} \rightarrow K_{\infty}$. Put $W(\epsilon)=K_{\infty} \cap X(\epsilon)$. By Proposition 2.3, we have $v(W(\epsilon)) / v$ $\left(B_{R}\left(x_{\infty}\right)\right) \geq 1-\Psi(\epsilon ; n, R, L)$. Fix $\alpha \in W(\epsilon), 0<t<<\min \{\eta, r(\alpha)\}$ and an isometric embedding $\gamma$ from $\left[0, \overline{z_{\infty}, \alpha}+\eta\right]$ to $Y$ with $\gamma(0)=z_{\infty}, \gamma\left(\overline{z_{\infty}, \alpha}\right)=$ $\alpha$. Let $\left\{\alpha_{i}\right\}_{i}$ be a sequence of points $\alpha_{i}$ in $K_{i}$ with $\alpha_{i} \rightarrow \alpha$. Define a Borel function $F_{i}$ on $B_{t}\left(\alpha_{i}\right) \backslash\left(C_{z_{i}} \cup\left\{z_{i}\right\}\right)$ by $F_{i}(\beta)=\left(f_{i} \circ \gamma_{\beta}\left(\overline{z_{i}, \beta}-\eta^{2}\right)-f_{i}(\beta)\right) /$ $\left(-\eta^{2}\right)$, where $\gamma_{\beta}$ is the minimal geodesic from $z_{i}$ to $\beta$. By an argument similar to the proof of Claim 3.19, we have

$$
\begin{aligned}
\frac{1}{\underline{\operatorname{vol}} B_{t}\left(\alpha_{i}\right)} \int_{B_{t}\left(\alpha_{i}\right)}\left|\left\langle d f_{i}, d r_{z_{i}}\right\rangle-F_{i}\right| d \underline{\mathrm{vol}} & \leq \eta^{2} \frac{C(n)}{\underline{\operatorname{vol} B_{10 t}\left(\alpha_{i}\right)}} \int_{B_{10 t}\left(\alpha_{i}\right)}\left|\operatorname{Hess}_{f_{i}}\right| d \underline{\mathrm{vol}} \\
& \leq \eta^{2} C(n) l \leq \Psi(\epsilon ; n)
\end{aligned}
$$

for every $i$. Fix $i_{0}$ with $\epsilon_{i}<<t$ for every $i \geq i_{0}$. We remark that $\overline{\phi_{i}\left(\beta_{i}\right), \alpha} \leq$ $t+\epsilon_{i} \leq \eta^{3}$ for every $i \geq i_{0}$ and every $\beta_{i} \in B_{t}\left(\alpha_{i}\right)$. Then, since

$$
\begin{aligned}
& \overline{z, \phi_{i}\left(\gamma_{\beta_{i}}\left(\overline{z_{i}, \beta_{i}}-\eta^{2}\right)\right)} \eta^{-2} d_{Y}+\overline{\phi_{i}\left(\gamma_{\beta_{i}}\left(\overline{z_{i}, \beta_{i}}-\eta^{2}\right)\right), \phi_{i}\left(\beta_{i}\right)} \eta^{-2} d_{Y}-\overline{z, \phi_{i}\left(\beta_{i}\right)} \eta^{-2} d_{Y} \\
& \quad<3 \epsilon_{i},
\end{aligned}
$$

we have

$$
\overline{z, \phi_{i}\left(\gamma_{\beta_{i}}\left(\overline{z_{i}, \beta_{i}}-\eta^{2}\right)\right)} \eta^{-2} d_{Y}+\overline{\phi_{i}\left(\gamma_{\beta_{i}}\left(\overline{z_{i}, \beta_{i}}-\eta^{2}\right)\right), \alpha} \eta^{-2} d_{Y}-\overline{z, \alpha} \eta^{-2} d_{Y}<5 \eta .
$$

Similarly, we have

$$
\begin{aligned}
& \overline{z, \phi_{i}\left(\gamma_{\beta_{i}}\left(\overline{z_{i}, \beta_{i}}-\eta^{2}\right)\right)} \eta^{-2} d_{Y}+\overline{\phi_{i}\left(\gamma_{\beta_{i}}\left(\overline{z_{i}, \beta_{i}}-\eta^{2}\right)\right), \gamma(\overline{z, \alpha}+\eta)} \eta^{-2} d_{Y} \\
& \quad-\overline{z, \gamma(\overline{z, \alpha}+\eta)} \eta^{-2} d_{Y}<5 \eta \\
& \overline{\phi_{i}\left(\gamma_{\beta_{i}}\left(\overline{z_{i}, \beta_{i}}-\eta^{2}\right)\right), \gamma(\overline{z, \alpha}+\eta)} \eta^{-2} d_{Y} \\
& \geq \eta^{-1}-\eta, \overline{\phi_{i}\left(\gamma_{\beta_{i}}\left(\overline{z_{i}, \beta_{i}}-\eta^{2}\right)\right), z} \eta^{-2} d_{Y} \geq \eta^{-1}-\eta
\end{aligned}
$$

and

$$
\overline{\phi_{i}\left(\gamma_{\beta_{i}}\left(\overline{z_{i}, \beta_{i}}-\eta^{2}\right)\right), \alpha} \eta^{-2} d_{Y}=1 \pm 5 \eta
$$


Therefore, by the splitting theorem on limit spaces, we have

$$
\overline{\phi_{i}\left(\gamma_{\beta_{i}}\left(\overline{z_{i}, \beta_{i}}-\eta^{2}\right)\right), \gamma\left(\overline{z, \alpha}-\eta^{2}\right)} \eta^{-2} d_{Y} \leq \Psi(\eta ; n)
$$

Thus, we have

$$
\begin{aligned}
\frac{f_{i}\left(\gamma_{\beta_{i}}\left(\overline{z_{i}, \beta_{i}}-\eta^{2}\right)\right)-f_{i}\left(\beta_{i}\right)}{-\eta^{2}} & =\frac{f_{\infty}\left(\phi_{i}\left(\gamma_{\beta_{i}}\left(\overline{z_{i}, \beta_{i}}-\eta^{2}\right)\right)\right)-f_{\infty}\left(\phi_{i}\left(\beta_{i}\right)\right)}{-\eta^{2}} \pm \frac{\epsilon_{i}}{\eta^{2}} \\
& =\frac{\left.f_{\infty}\left(\gamma\left(\overline{z, \alpha}-\eta^{2}\right)\right)\right)-f_{\infty}(\alpha)}{-\eta^{2}} \pm \Psi(\eta ; n, L) \\
& =\left\langle d r_{z}, d f_{\infty}\right\rangle(\alpha) \pm \Psi(\eta ; n, L) .
\end{aligned}
$$

Especially, we have

$$
\frac{1}{\underline{\mathrm{vol}} B_{t}\left(\alpha_{i}\right)} \int_{B_{t}\left(\alpha_{i}\right)}\left|F_{i}-\left\langle d r_{z}, d f_{\infty}\right\rangle(\alpha)\right| d \underline{\mathrm{vol}} \leq \Psi(\eta ; n, L)
$$

for every $i \geq i_{0}$. Put $W=\bigcap_{N_{1} \in \mathbf{N}}\left(\bigcup_{N_{2} \geq N_{1}} W\left(N_{2}^{-1}\right)\right)$. Then we have $v\left(B_{R}\left(x_{\infty}\right) \backslash W\right)=0$. Moreover, by the argument above, $\left\langle d r_{z_{i}}, d f_{i}\right\rangle$ converges strongly to $\left\langle d r_{w}, d f_{\infty}\right\rangle$ at every $w_{\infty} \in W$.

Remark 4.2. We shall introduce the following important method to obtain a uniformly $L^{2}$-Hessian estimates by using cut-off functions with good properties constructed by Cheeger-Colding: Let $(M, m)$ be a pointed $n$-dimensional complete Riemannian manifold with $\operatorname{Ric}_{M} \geq-(n-1), R$ a positive number and $f$ a $C^{2}$-function on $B_{R}(m)$. Assume that there exists $L \geq 1$ such that

$$
|\nabla f|_{L^{\infty}\left(B_{R}(m)\right)}+\int_{B_{R}(m)}|\Delta f|^{2} d \underline{\mathrm{vol}} \leq L
$$

Then, we have

$$
\int_{B_{r}(m)}\left|\operatorname{Hess}_{f}\right|^{2} d \underline{\mathrm{vol}}<C(n, r, R, L)
$$

for every $0<r<R$. The proof is as follows. By the standard smoothing argument, without loss of generality, we can assume that $f$ is a smooth function. By [2, Theorem 8.16], there exists a smooth function $\phi$ on $M$ such that $0 \leq \phi \leq 1,\left.\phi\right|_{B_{r}(m)}=1, \operatorname{supp} \phi \subset B_{R}(m),|\nabla \phi| \leq C(n, r, R)$ and 
$|\Delta \phi| \leq C(n, r, R)$. By Bochner's formula, we have

$$
-\frac{1}{2} \Delta|\nabla(\phi f)|^{2} \geq\left|\operatorname{Hess}_{\phi f}\right|^{2}-\langle\nabla \Delta(\phi f), \nabla(\phi f)\rangle-(n-1)|\nabla(\phi f)|^{2} .
$$

Thus, we have

$$
\begin{aligned}
\int_{B_{r}(m)}\left|\operatorname{Hess}_{f}\right|^{2} d \underline{\mathrm{vol}} \leq & \int_{B_{R}(m)}\left|\operatorname{Hess}_{\phi f}\right|^{2} d \underline{\mathrm{vol}} \\
\leq & \int_{B_{R}(m)}(\Delta(\phi f))^{2} d \underline{\mathrm{vol}}+C(n, R, L) \\
\leq & \int_{B_{R}(m)}\left((f \Delta \phi)^{2}+(\phi \Delta f)^{2}+|\langle\nabla f, \nabla \phi\rangle|^{2}\right) d \underline{\mathrm{vol}} \\
& +C(n, R, L) \\
\leq & C(n, r, R, L) .
\end{aligned}
$$

This observation performs a crucial role to study limit functions of harmonic functions in Subsection 4.4 .

The following proposition follows from Corollary 3.1 directly.

Proposition 4.3. Let $\left\{\left(M_{i}, m_{i}\right)\right\}_{i}$ be a sequence of pointed $n$-dimensional complete Riemannian manifolds with $\operatorname{Ric}_{M_{i}} \geq-(n-1),(Y, y, v)$ a Ricci limit space of $\left\{\left(M_{i}, m_{i}, \underline{\mathrm{vol}}\right)\right\}_{i}, w_{\infty}^{1}, w_{\infty}^{2}$ points in $Y$, and $w_{i}^{1}, w_{i}^{2}$ points in $M_{i}$ for every $i$, satisfying that $w_{i}^{j} \rightarrow w_{\infty}^{j}$ for every $j$. Then $\left\langle d r_{w_{i}^{1}}, d r_{w_{i}^{2}}\right\rangle$ converges strongly to $\left\langle d r_{w_{\infty}^{1}}, d r_{w_{\infty}^{2}}\right\rangle$ at every $z \in Y \backslash\left(C_{w_{\infty}^{1}} \cup C_{w_{\infty}^{2}} \cup\left\{w_{\infty}^{1}, w_{\infty}^{2}\right\}\right)$.

\subsection{Pointwise weak convergence of $L^{\infty}$-functions}

Our aims in this subsection are to give the following notion and its fundamental properties.

Definition 4.2 (Pointwise weak convergence of $L^{\infty}$-functions). Let $R$ be a positive number, $w_{\infty}$ a point in $B_{R}\left(x_{\infty}\right)$ and $\left\{f_{i}\right\}_{1 \leq i \leq \infty}$ a sequence of $L^{\infty}$-functions $f_{i}$ on $B_{R}\left(x_{i}\right)$ with $\sup _{i}\left|f_{i}\right|_{L^{\infty}\left(B_{R}\left(x_{i}\right)\right)}<\infty$. We say that $f_{i}$ converges weakly to $f_{\infty}$ at $w_{\infty}$ if for every $\epsilon>0$, there exists $r>0$ such that

$$
\limsup _{i \rightarrow \infty}\left|\frac{1}{v_{i}\left(B_{t}\left(w_{i}\right)\right)} \int_{B_{t}\left(w_{i}\right)} f_{i} d v_{i}-\frac{1}{v_{\infty}\left(B_{t}\left(w_{\infty}\right)\right)} \int_{B_{t}\left(w_{\infty}\right)} f_{\infty} d v_{\infty}\right| \leq \epsilon
$$

for every $0<t<r$ and every $w_{i} \rightarrow w_{\infty}$. 
It is clear that if $f_{i}$ converges strongly to $f_{\infty}$ at $w_{\infty}$, then $f_{i}$ converges weakly to $f_{\infty}$ at $w_{\infty}$. We skip the proof of the next proposition because it is not difficult to check it.

Proposition 4.4 (Linearlity of weak convergence). Let $R$ be a positive number, $w_{\infty}$ a point in $B_{R}\left(x_{\infty}\right)$ and $a_{i}, b_{i}, c_{i}, d_{i} L^{\infty}$-functions on $B_{R}\left(x_{i}\right)$ for every $1 \leq i \leq \infty$ with $\sup _{i}\left(\left|a_{i}\right|+\left|b_{i}\right|+\left|c_{i}\right|+\left|d_{i}\right|\right)_{L^{\infty}\left(B_{R}\left(x_{i}\right)\right)}<\infty$. Assume that $a_{i}, b_{i}$ converge strongly to $a_{\infty}, b_{\infty}$ at $w_{\infty}$, respectively, and that $c_{i}, d_{i}$ converge weakly to $c_{\infty}, d_{\infty}$ at $w_{\infty}$, respectively. Then $a_{i} c_{i}+b_{i} d_{i}$ converges weakly to $a_{\infty} c_{\infty}+b_{\infty} d_{\infty}$ at $w_{\infty}$.

Proposition 4.5. Let $\left\{A_{i}\right\}_{1 \leq i \leq \infty}$ be a sequnece of Borel subsets $A_{i}$ of $B_{R}\left(x_{i}\right)$ and $w_{\infty}$ a point in Leb $A_{\infty}$. Assume that $1_{A_{i}}$ converges weakly to $1_{A_{\infty}}$ at $w_{\infty}$. Then $1_{A_{i}}$ converges strongly to $1_{A_{\infty}}$ at $w_{\infty}$.

Proof. Fix $\epsilon>0$. Let $\left\{w_{i}\right\}_{i}$ be a sequence of points $w_{i}$ in $Z_{i}$ satisfying $w_{i} \rightarrow$ $w_{\infty}$. There exists $r>0$ such that $v_{\infty}\left(B_{t}\left(w_{\infty}\right) \cap A_{\infty}\right) / v_{\infty}\left(B_{t}\left(w_{\infty}\right)\right) \geq 1-\epsilon$ and

$$
\limsup _{i \rightarrow \infty}\left|\frac{1}{v_{i}\left(B_{t}\left(w_{i}\right)\right)} \int_{B_{t}\left(w_{i}\right)} 1_{A_{i}} d v_{i}-\frac{1}{v_{\infty}\left(B_{t}\left(w_{\infty}\right)\right)} \int_{B_{t}\left(w_{\infty}\right)} 1_{A_{\infty}} d v_{\infty}\right|<\epsilon
$$

for every $0<t<r$. Fix $0<t<r$. Then we have

$$
\begin{aligned}
& \frac{1}{v_{i}\left(B_{t}\left(w_{i}\right)\right)} \int_{B_{t}\left(w_{i}\right)}\left|1_{A_{i}}-\frac{1}{v_{\infty}\left(B_{t}\left(w_{\infty}\right)\right)} \int_{B_{t}\left(w_{\infty}\right)} 1_{A_{\infty}} d v_{\infty}\right| d v_{i} \\
& \leq \frac{1}{v_{i}\left(B_{t}\left(w_{i}\right)\right)} \int_{B_{t}\left(w_{i}\right)}\left|1_{A_{i}}-\frac{1}{v_{i}\left(B_{t}\left(w_{i}\right)\right)} \int_{B_{t}\left(w_{i}\right)} 1_{A_{i}} d v_{i}\right| d v_{i}+\epsilon \\
&= \frac{1}{v_{i}\left(B_{t}\left(w_{i}\right)\right)} \int_{A_{i}} \frac{v_{i}\left(B_{t}\left(w_{i}\right) \backslash A_{i}\right)}{v_{i}\left(B_{t}\left(w_{i}\right)\right)} d v_{i}+\frac{1}{v_{i}\left(B_{t}\left(w_{i}\right)\right)} \\
& \times \int_{B_{t}\left(w_{i}\right) \backslash A_{i}} \frac{v_{i}\left(A_{i}\right)}{v_{i}\left(B_{t}\left(w_{i}\right)\right)} d v_{i}+\epsilon \\
& \leq 2 \frac{v_{i}\left(B_{t}\left(w_{i}\right) \backslash A_{i}\right)}{v_{i}\left(B_{t}\left(w_{i}\right)\right)}+\epsilon<2 \frac{v_{\infty}\left(B_{t}\left(w_{\infty}\right) \backslash A_{\infty}\right)}{v_{\infty}\left(B_{t}\left(w_{\infty}\right)\right)}+2 \epsilon<5 \epsilon .
\end{aligned}
$$

for every sufficiently large $i$. Similarly, we have

$$
\frac{1}{v_{\infty}\left(B_{t}\left(w_{\infty}\right)\right)} \int_{B_{t}\left(w_{\infty}\right)}\left|1_{A_{\infty}}-\frac{1}{v_{i}\left(B_{t}\left(w_{i}\right)\right)} \int_{B_{t}\left(w_{i}\right)} 1_{A_{i}} d v_{i}\right| d v_{\infty}<5 \epsilon
$$

for every sufficiently large $i$. Thus, we have the assertion. 
The next proposition follows from an argument similar to the proof of Proposition 2.3:

Proposition 4.6. Let $R$ be a positive number, $\left\{K_{i}\right\}_{1 \leq i \leq \infty}$ a sequence of Borel subsets $K_{i}$ of $\bar{B}_{R}\left(x_{i}\right)$, and $\left\{f_{i}\right\}_{1 \leq i \leq \infty}$ of non-negative valued $L^{\infty}$. functions $f_{i}$ on $\bar{B}_{R}\left(x_{i}\right)$ with $\sup _{i}\left|f_{i}\right|_{L^{\infty}\left(B_{R}\left(x_{i}\right)\right)}<\infty$. Assume that $K_{\infty}$ is compact, $\lim \sup _{i \rightarrow \infty}^{G H} K_{i} \subset K_{\infty}$ and that $f_{i}$ converges weakly to $f_{\infty}$ at a.e. $w \in K_{\infty}$. Then, we have

$$
\limsup _{i \rightarrow \infty} \int_{K_{i}} f_{i} d v_{i} \leq \int_{K_{\infty}} f_{\infty} d v_{\infty}
$$

We shall give a fundamental result about this weak convergence:

Proposition 4.7. Let $R$ be a positive number, $\left\{A_{i}\right\}_{1 \leq i \leq \infty}$ a sequence of Borel subsets $A_{i}$ of $\bar{B}_{R}\left(x_{i}\right)$, and $\left\{f_{i}\right\}_{1 \leq i \leq \infty}$ of $L^{\infty}$-functions $f_{i}$ on $\bar{B}_{R}\left(x_{i}\right)$ with $\sup _{i}\left|f_{i}\right|_{L^{\infty}\left(B_{R}\left(x_{i}\right)\right)}<\infty$. Assume that $1_{A_{i}}$ converges weakly to $1_{A_{\infty}}$ at a.e. $w \in B_{R}\left(x_{\infty}\right)$ and that $f_{i}$ converges weakly to $f_{\infty}$ at a.e. $w \in A_{\infty}$. Then, we have

$$
\lim _{i \rightarrow \infty} \int_{A_{i}} f_{i} d v_{i}=\int_{A_{\infty}} f_{\infty} d v_{\infty}
$$

Proof. It follows from (the proof of ) Propositions 4.4 and 4.5 that $f_{i} 1_{A_{i}}$ converges weakly to $f_{\infty} 1_{A_{\infty}}$ at a.e. $w_{\infty} \in B_{R}\left(x_{\infty}\right)$. Thus, without loss of generality, we can assume that $A_{i}=B_{R}\left(x_{i}\right)$ for every $1 \leq i \leq \infty$. Fix $\epsilon>0$. Let $L \geq 1$ with $\sup _{i}\left|f_{i}\right|_{L^{\infty}\left(B_{R}\left(x_{i}\right)\right)}+v_{\infty}\left(B_{R}\left(x_{\infty}\right)\right)<L$. There exists a Borel subset $\hat{K}_{\infty}$ of $B_{R}\left(x_{\infty}\right)$ such that $v\left(B_{R}\left(x_{\infty}\right) \backslash \hat{K}_{\infty}\right)=0$ and that for every $w_{\infty} \in \hat{K}_{\infty}$, there exists $t_{w_{\infty}}>0$ such that $\bar{B}_{10 t_{w_{\infty}}}\left(w_{\infty}\right) \subset B_{R}(x)$ and

$$
\limsup _{i \rightarrow \infty}\left|\frac{1}{v_{i}\left(B_{s}\left(w_{i}\right)\right)} \int_{B_{s}\left(w_{i}\right)} f_{i} d v_{i}-\frac{1}{v_{\infty}\left(B_{s}\left(w_{\infty}\right)\right)} \int_{B_{s}\left(w_{\infty}\right)} f_{\infty} d v_{\infty}\right|<\epsilon
$$

for every $0<s<t_{w_{\infty}}$ and every $w_{i} \rightarrow w_{\infty}$. By Lemma 2.2, there exists a pairwise disjoint collection $\left\{\bar{B}_{r_{i}}\left(x_{i}\right)\right\}_{i}$ such that $x_{i} \in \hat{K}_{\infty}, r_{i}<<t_{x_{i}}$, and $\hat{K}_{\infty} \backslash \bigcup_{i=1}^{N} \bar{B}_{r_{i}}\left(x_{i}\right) \subset \bigcup_{i=N+1}^{\infty} \bar{B}_{5 r_{i}}\left(x_{i}\right)$ for every $N$. Fix $N$ satisfying $\sum_{i=N+1}^{\infty}$ $v_{\infty}\left(B_{r_{i}}\left(x_{i}\right)\right)<\epsilon$. Then, we have $\sum_{i=N+1}^{\infty} v_{\infty}\left(B_{5 r_{i}}\left(x_{i}\right)\right)<2^{5 \kappa(1)} \epsilon$. For every 
$i, j$, let $x_{i}(j)$ be a point in $Z_{j}$ satisfying $x_{i}(j) \rightarrow x_{i}$. Then we have

$$
\begin{aligned}
\int_{B_{R}\left(x_{\infty}\right)} f_{\infty} d v_{\infty}= & \sum_{i=1}^{N} \int_{B_{r_{i}}\left(x_{i}\right)} f_{\infty} d v_{\infty} \pm \Psi(\epsilon ; \kappa(1), L) \\
= & \sum_{i=1}^{N} \int_{B_{r_{i}}\left(x_{i}(j)\right)} f_{j} d v_{j} \pm \Psi(\epsilon ; \kappa(1), L) \\
= & \int_{B_{R}\left(x_{j}\right)} f_{j} d v_{j} \pm\left(\int_{B_{R}\left(x_{j}\right) \backslash \bigcup_{i=1}^{N} \bar{B}_{r_{i}}\left(x_{i}(j)\right)}\left|f_{j}\right| d v_{j}\right. \\
& +\Psi(\epsilon ; \kappa(1), L))
\end{aligned}
$$

for every sufficiently large $j$. On the other hand, by Propositions 2.1 and 2.3, we have

$$
\begin{aligned}
\limsup _{j \rightarrow \infty} \int_{B_{R}\left(x_{j}\right) \backslash \bigcup_{i=1}^{N} \bar{B}_{r_{i}}\left(x_{i}(j)\right)}\left|f_{j}\right| d v_{j} & \leq L \limsup _{j \rightarrow \infty} v_{j}\left(\bar{B}_{R}\left(x_{j}\right) \backslash \bigcup_{i=1}^{N} B_{r_{i}}\left(x_{i}(j)\right)\right) \\
& \leq L v_{\infty}\left(\hat{K}_{\infty} \backslash \bigcup_{i=1}^{N} B_{r_{i}}\left(x_{i}\right)\right) \\
& \leq \Psi(\epsilon ; \kappa(1), L) .
\end{aligned}
$$

Therefore, we have the assertion.

Next corollary follows from Proposition 4.7 directly:

Corollary 4.1. Let $R$ be a positive number, $N$ a positive integer, $\left\{r_{j}\right\}_{1 \leq j \leq N}$ a collection of positive numbers, $\left\{z_{j}\right\}_{1 \leq j \leq N}$ of points in $Y$, and $\left\{f_{i}\right\}_{1 \leq i \leq \infty}$ a sequence of $L^{\infty}$-functions $f_{i}$ on $B_{R}\left(x_{i}\right)$ with $\sup _{i}\left|f_{i}\right|_{L^{\infty}\left(B_{R}\left(x_{i}\right)\right)}$ $<\infty$. Assume that $f_{i}$ converges weakly to $f_{\infty}$ at a.e. $w \in B_{R}\left(x_{\infty}\right) \backslash \bigcup_{i=1}^{N} B_{r_{i}}$ $\left(z_{i}\right)$. Then, we have

$$
\lim _{j \rightarrow \infty} \int_{B_{R}\left(x_{j}\right) \backslash \bigcup_{i=1}^{N} B_{r_{i}}\left(z_{i}(j)\right)} f_{j} d v_{j}=\int_{B_{R}\left(x_{\infty}\right) \backslash \bigcup_{i=1}^{N} B_{r_{i}}\left(z_{i}\right)} f_{\infty} d v_{\infty}
$$

for every $z_{i}(j) \rightarrow z_{i}$. 


\subsection{Convergence of the differentials of Lipschitz functions}

A purpose of this subsection is to give the definition of a convergence: $d f_{i} \rightarrow$ $d f_{\infty}$. See Definition 1.1 or Definition 4.4. Throughout this subsection, we fix the following situation:

1. Let $\left\{\left(M_{i}, m_{i}\right)\right\}_{1 \leq i<\infty}$ be a sequence of pointed $n$-dimensional complete Riemannian manifolds with $\operatorname{Ric}_{M_{i}} \geq-(n-1)$.

2. Let $(Y, y, v)$ be a Ricci limit space of $\left\{\left(M_{i}, m_{i}, \underline{\mathrm{vol}}\right)\right\}_{i}$.

3. Let $R$ be a positive number, $\left\{x_{i}\right\}_{1 \leq i<\infty}$ a sequence of points $x_{i}$ in $M_{i}$, and $x_{\infty}$ a point in $Y$ satisfying $x_{i} \rightarrow x_{\infty}$.

4. Let $\left\{f_{i}\right\}_{1 \leq i \leq \infty}$ be a sequence of Lipschitz functions $f_{i}$ on $B_{R}\left(x_{i}\right)$ with $\sup _{i}\left(\operatorname{Lip} f_{i}+\left|f_{i}\right|_{L^{\infty}\left(B_{R}\left(x_{i}\right)\right)}\right)<\infty$.

In this setting, we recall that $f_{i}$ converges to $f_{\infty}$ at $w_{\infty} \in B_{R}\left(x_{\infty}\right)$ if $f_{i}\left(w_{i}\right) \rightarrow$ $f_{\infty}\left(w_{\infty}\right)$ holds for every $w_{i} \rightarrow w_{\infty}$. See Section 1.2 . We denote it by $f_{i} \rightarrow f_{\infty}$ at $w_{\infty}$. We remark that it is easy to check that the following conditions are equivalent:

1. $f_{i}$ converges strongly to $f_{\infty}$ at $w_{\infty}$.

2. $f_{i} \rightarrow f_{\infty}$ at $w_{\infty}$.

3. $f_{i}$ converges weakly to $f_{\infty}$ at $w_{\infty}$.

We shall consider a convergence of the $L^{2}$-energy of Lipschitz functions. 0

Definition 4.3 (Pointwise upper semicontinuity of $L^{2}$-energy). We say that $L^{2}$-energy of $\left\{f_{i}\right\}_{i}$ are upper semicontinuous at $w_{\infty} \in B_{R}\left(x_{\infty}\right)$ if for every $\epsilon>0$, there exists $r>0$ such that

$\limsup _{i \rightarrow \infty} \frac{1}{\underline{\operatorname{vol}} B_{t}\left(w_{i}\right)} \int_{B_{t}\left(w_{i}\right)}\left(\operatorname{Lip} f_{i}\right)^{2} d \underline{\mathrm{vol}} \leq \frac{1}{v\left(B_{t}\left(w_{\infty}\right)\right)} \int_{B_{t}\left(w_{\infty}\right)}\left(\operatorname{Lip} f_{\infty}\right)^{2} d v+\epsilon$ for every $0<t<r$ and every $w_{i} \rightarrow w_{\infty}$.

By the definition, if $\left(\operatorname{Lip} f_{i}\right)^{2}$ converges weakly to $\left(\operatorname{Lip} f_{\infty}\right)^{2}$ at $w_{\infty}$, then $L^{2}$-energy of $\left\{f_{i}\right\}_{i}$ are upper semicontinuous at $w_{\infty}$. We shall give the definition of a convergence of the differentials of Lipschitz functions:

Definition 4.4 (Convergence of the differentials of Lipschitz functions). We say that $d f_{i}$ converges to $d f_{\infty}$ at $w_{\infty} \in B_{R}\left(x_{\infty}\right)$ if the following properties hold: 
1. $\left\langle d r_{z_{i}}, d f_{i}\right\rangle$ converges weakly to $\left\langle d r_{z_{\infty}}, d f_{\infty}\right\rangle$ at $w_{\infty}$ for every $z_{i} \rightarrow z_{\infty}$;

2. $L^{2}$-energy of $\left\{f_{i}\right\}_{i}$ are upper semicontinuous at $w_{\infty}$.

Then we denote it by $d f_{i} \rightarrow d f_{\infty}$ at $w_{\infty}$. Moreover, for a subset $A$ of $B_{R}\left(x_{\infty}\right)$, if $f_{i} \rightarrow f_{\infty}$ and $d f_{i} \rightarrow d f_{\infty}$ at every $a \in A$, then we denote it by $\left(f_{i}, d f_{i}\right) \rightarrow$ $\left(f_{\infty}, d f_{\infty}\right)$ on $A$.

Proposition 4.8. Let $w_{i}$ be a point in $M_{i}$ for every $i<\infty$, and $w_{\infty}$ a point in $Y$ with $w_{i} \rightarrow w_{\infty}$. Then we have $\left(r_{w_{i}}, d r_{w_{i}}\right) \rightarrow\left(r_{w_{\infty}}, d r_{w_{\infty}}\right)$ on $Y$.

Proof. It follows from Propositions 4.3 and 4.7 directly.

The following theorem is the main result in this subsection:

Theorem 4.1. Let $\left\{g_{i}\right\}_{1 \leq i \leq \infty}$ be a sequence of Lipschitz functions $g_{i}$ on $B_{R}\left(x_{i}\right)$, and $A$ a Borel subset of $B_{R}\left(x_{\infty}\right)$. Assume that $\sup _{i}\left(\mathbf{L i p} g_{i}+\right.$ $\left.\left|g_{i}\right|_{L^{\infty}\left(B_{R}\left(x_{i}\right)\right)}\right)<\infty, d f_{i} \rightarrow d f_{\infty}$ and $d g_{i} \rightarrow d g_{\infty}$ on $A$. Then, $\left\langle d f_{i}, d g_{i}\right\rangle$ converges strongly to $\left\langle d f_{\infty}, d g_{\infty}\right\rangle$ at a.e. $w_{\infty} \in A$.

Proof. By Theorem 3.1 and Lemma 3.15, there exist collections of Borel subset $\left\{A_{j}\right\}_{j}$ of $A$, of positive integers $\left\{k_{j}\right\}_{j}$ with $1 \leq k_{j} \leq n$, and of points $\left\{x_{l}^{j}\right\}_{j, 1 \leq l \leq k_{j}}$ in $Y$ such that the following properties hold:

1. $v\left(A \backslash \bigcup_{j=1}^{\infty} A_{j}\right)=0$ and $A_{j} \subset Y \backslash \bigcup_{l=1}^{k_{j}}\left(C_{x_{l}^{j}} \cup\left\{x_{l}^{j}\right\}\right)$ for every $j$.

2. For every $w \in A_{j}$, there exists $a_{1}^{j}, \ldots, a_{k_{j}}^{j}, b_{1}^{j}, \ldots, b_{k_{j}}^{j} \in \mathbf{R}$ such that

$$
\begin{aligned}
& \lim _{r \rightarrow 0} \frac{1}{v\left(B_{r}\left(w_{\infty}\right)\right)} \int_{B_{r}\left(w_{\infty}\right)}\left|d f_{\infty}-d\left(\sum_{l=1}^{k_{j}} a_{l}^{j} r_{x_{l}^{j}}\right)\right|^{2} \\
& +\left|d g_{\infty}-d\left(\sum_{l=1}^{k_{j}} b_{l}^{j} r_{x_{l}^{j}}\right)\right|^{2} d v=0
\end{aligned}
$$

Fix $j$ and $w_{\infty} \in A_{j}$. Let $a_{1}^{j}, \ldots, a_{k_{j}}^{j}, b_{1}^{j}, \ldots, b_{k_{j}}^{j} \in \mathbf{R}$ as above, and $L \geq 1$ with $\sup _{i}\left(\operatorname{Lip} f_{i}+\operatorname{Lip} g_{i}\right)+\sum_{l=1}^{k_{j}}\left(\left(a_{l}^{j}\right)^{2}+\left(b_{l}^{j}\right)^{2}\right) \leq L$. Take $\tau>0$ with $w \in$ $\bigcup_{l=1}^{k_{j}}\left(D_{x_{l}^{j}}^{\tau} \backslash B_{\tau}\left(x_{l}^{j}\right)\right)$. Let $x_{l}^{j}(i) \rightarrow x_{l}^{j}$ and $w_{i} \rightarrow w_{\infty}$. Fix $\epsilon>0$. Then, there 
exists $r>0$ such that

$$
\begin{aligned}
& \frac{1}{v\left(B_{t}\left(w_{\infty}\right)\right)} \int_{B_{t}\left(w_{\infty}\right)}\left|d f_{\infty}-d\left(\sum_{l=1}^{k_{j}} a_{l}^{j} r_{x_{l}^{j}}\right)\right|^{2}+\left|d g_{\infty}-d\left(\sum_{l=1}^{k_{j}} b_{l}^{j} r_{x_{l}^{j}}\right)\right|^{2} d v \\
& \quad \leq \epsilon \\
& \quad \limsup _{i \rightarrow \infty} \frac{1}{\quad \underline{\operatorname{vol}} B_{t}\left(w_{i}\right)} \int_{B_{t}\left(w_{i}\right)}\left(\operatorname{Lip} f_{i}\right)^{2} d \underline{\mathrm{vol}} \leq \frac{1}{v\left(B_{t}\left(w_{\infty}\right)\right)} \\
& \quad \times \int_{B_{t}\left(w_{\infty}\right)}\left(\operatorname{Lip} f_{\infty}\right)^{2} d v+\epsilon \\
& \limsup _{i \rightarrow \infty} \frac{1}{\underline{\operatorname{vol}} B_{t}\left(w_{i}\right)} \int_{B_{t}\left(w_{i}\right)}\left(\operatorname{Lip} g_{i}\right)^{2} d \underline{\operatorname{vol}} \leq \frac{1}{v\left(B_{t}\left(w_{\infty}\right)\right)}
\end{aligned}
$$$$
\times \int_{B_{t}\left(w_{\infty}\right)}\left(\operatorname{Lip} g_{\infty}\right)^{2} d v+\epsilon
$$$$
\limsup _{i \rightarrow \infty} \mid \frac{1}{\underline{\operatorname{vol}} B_{t}\left(w_{i}\right)} \int_{B_{t}\left(w_{i}\right)}\left\langle d f_{i}, d r_{x_{l}^{j}(i)}\right\rangle d \underline{\operatorname{vol}}-\frac{1}{v\left(B_{t}\left(w_{\infty}\right)\right)}
$$$$
\times \int_{B_{t}\left(w_{\infty}\right)}\left\langle d f_{\infty}, d r_{x_{l}^{j}}\right\rangle d v \mid<\epsilon
$$

and

$$
\begin{aligned}
& \limsup _{i \rightarrow \infty} \mid \frac{1}{\underline{\operatorname{vol}} B_{t}\left(w_{i}\right)} \int_{B_{t}\left(w_{i}\right)}\left\langle d g_{i}, d r_{x_{l}^{j}(i)}\right\rangle d \underline{\mathrm{vol}} \\
& -\times \frac{1}{v\left(B_{t}\left(w_{\infty}\right)\right)} \int_{B_{t}\left(w_{\infty}\right)}\left\langle d g_{\infty}, d r_{x_{l}^{j}}\right\rangle d v \mid<\epsilon
\end{aligned}
$$

for every $l$ and every $0<t<r$. Fix $0<t<<\min \{r, \epsilon, \tau\}$. Then, by Corollary 3.1 , we have

$$
\begin{aligned}
& \frac{1}{v\left(B_{t}\left(w_{\infty}\right)\right)} \int_{B_{t}\left(w_{\infty}\right)} \mid\left\langle d f_{\infty}, d g_{\infty}\right\rangle-\frac{1}{v\left(B_{t}\left(w_{\infty}\right)\right)} \\
& \int_{B_{t}\left(w_{\infty}\right)}\left\langle d\left(\sum_{l=1}^{k_{j}} a_{l}^{j} r_{x_{l}^{j}}\right), d\left(\sum_{l=1}^{k_{j}} b_{l}^{j} r_{x_{l}^{j}}\right)\right\rangle d v \mid d v \leq \Psi(\epsilon ; n, L)
\end{aligned}
$$


and

$$
\begin{aligned}
& \frac{1}{v\left(B_{t}\left(w_{\infty}\right)\right)} \int_{B_{t}\left(w_{\infty}\right)}\left|\left\langle d f_{\infty}, d g_{\infty}\right\rangle-\frac{1}{v\left(B_{t}(w)\right)} \int_{B_{t}(w)}\left\langle d f_{\infty}, d g_{\infty}\right\rangle d v\right| d v \\
& =\frac{1}{v\left(B_{t}\left(w_{\infty}\right)\right)} \int_{B_{t}\left(w_{\infty}\right)} \mid\left\langle d\left(\sum_{l=1}^{k_{j}} a_{l}^{j} r_{x_{l}^{j}}\right), d\left(\sum_{l=1}^{k_{j}} b_{l}^{j} r_{x_{l}^{j}}\right)\right\rangle \\
& \quad-\frac{1}{v\left(B_{t}\left(w_{\infty}\right)\right)} \int_{B_{t}\left(w_{\infty}\right)}\left\langle d\left(\sum_{l=1}^{k_{j}} a_{l}^{j} r_{x_{l}^{j}}\right), d\left(\sum_{l=1}^{k_{j}} b_{l}^{j} r_{x_{l}^{j}}\right)\right\rangle d v \mid d v \\
& \quad \pm \Psi(\epsilon ; n, L)=\Psi(\epsilon ; n, L) .
\end{aligned}
$$

On the other hand, we have

$$
\begin{aligned}
& \frac{1}{\underline{\operatorname{vol}} B_{t}\left(w_{i}\right)} \int_{B_{t}\left(w_{i}\right)}\left|d f_{i}-d\left(\sum_{l=1}^{k_{j}} a_{l}^{j} r_{x_{l}^{j}(i)}\right)\right|^{2} d \underline{\text { vol }} \\
& =\frac{1}{\underline{\operatorname{vol}} B_{t}\left(w_{i}\right)} \int_{B_{t}\left(w_{i}\right)}\left|d f_{i}\right|^{2} d \underline{\mathrm{vol}}-\sum_{l=1}^{k_{j}} \frac{a_{l}^{j}}{\underline{\operatorname{vol}} B_{t}\left(w_{i}\right)} \int_{B_{t}\left(w_{i}\right)}\left\langle d f_{i}, d r_{x_{l}^{j}(i)}\right\rangle d \underline{\mathrm{vol}} \\
& +\sum_{l, \hat{l}} \frac{a_{l}^{j} a_{\hat{l}}^{j}}{\underline{\operatorname{vol}} B_{t}\left(w_{i}\right)} \int_{B_{t}\left(w_{i}\right)}\left\langle d r_{x_{l}^{j}(i)}, d r_{x_{\hat{\imath}}^{j}(i)}\right\rangle d \underline{\mathrm{vol}} \\
& \leq \frac{1}{v\left(B_{t}\left(w_{\infty}\right)\right)} \int_{B_{t}\left(w_{\infty}\right)}\left|d f_{\infty}\right|^{2} d v-\sum_{l=1}^{k} \frac{a_{l}^{j}}{v\left(B_{t}\left(w_{\infty}\right)\right)} \int_{B_{t}\left(w_{\infty}\right)}\left\langle d f_{\infty}, d r_{x_{l}^{j}}\right\rangle d v \\
& +\sum_{l, \hat{l}} \frac{a_{l}^{j} a_{\hat{l}}^{j}}{v\left(B_{t}\left(w_{\infty}\right)\right)} \int_{B_{t}\left(w_{\infty}\right)}\left\langle d r_{x_{l}^{j}}, d r_{x_{\hat{\imath}}^{j}}\right\rangle d v+\Psi(\epsilon ; n, L) \\
& =\frac{1}{v\left(B_{t}\left(w_{\infty}\right)\right)} \int_{B_{t}\left(w_{\infty}\right)}\left|d f_{\infty}-d\left(\sum_{l=1}^{k_{j}} a_{l}^{j} r_{x_{l}^{j}}\right)\right|^{2} d v+\Psi(\epsilon ; n, L) \\
& \leq \Psi(\epsilon ; n, L)
\end{aligned}
$$

for every sufficiently large $i$. Similarly, we have

$$
\frac{1}{\underline{\mathrm{vol}} B_{t}\left(w_{i}\right)} \int_{B_{t}\left(w_{i}\right)}\left|d g_{i}-d\left(\sum_{l=1}^{k_{j}} b_{l}^{j} r_{x_{l}^{j}(i)}\right)\right|^{2} d \underline{\mathrm{vol}} \leq \Psi(\epsilon ; n, L)
$$


for every sufficiently large $i$. Especially, we have

$$
\begin{aligned}
& \frac{1}{\underline{\operatorname{vol}} B_{t}\left(w_{i}\right)} \int_{B_{t}\left(w_{i}\right)} \mid\left\langle d f_{i}, d g_{i}\right\rangle-\frac{1}{\underline{\operatorname{vol}} B_{t}\left(w_{i}\right)} \\
& \quad \times \int_{B_{t}\left(w_{i}\right)}\left\langle d\left(\sum_{l=1}^{k_{j}} a_{l}^{j} r_{x_{l}^{j}(i)}\right), d\left(\sum_{l=1}^{k_{j}} b_{l}^{j} r_{x_{l}^{j}(i)}\right)\right\rangle d \underline{\mathrm{vol}} \mid d \underline{\mathrm{vol}} \leq \Psi(\epsilon ; n, L) .
\end{aligned}
$$

Therefore, by Corollary 3.1, we have the assertion.

Corollary 4.2. Let $\Omega$ be a non-empty open subset of $B_{R}\left(x_{\infty}\right)$. Assume that $d f_{i} \rightarrow d f_{\infty}$ at a.e. $w \in \Omega$. Then $d f_{i} \rightarrow d f_{\infty}$ on $\Omega$.

Proof. The assertion follows from Proposition 4.7 and Theorem 4.1.

Corollary 4.3. Let $\left\{g_{i}\right\}_{1 \leq i \leq \infty}$ be a sequence of Lipschitz functions $g_{i}$ on $B_{R}\left(x_{i}\right)$ with $\sup _{i}\left(\mathbf{L i p} g_{i}+\left|g_{i}\right|_{L^{\infty}\left(B_{R}\left(x_{i}\right)\right)}\right)<\infty$, and $A$ a Borel subset of $B_{R}\left(x_{\infty}\right)$. Assume that $\left(f_{i}, d f_{i}\right) \rightarrow\left(f_{\infty}, d f_{\infty}\right)$ and $\left(g_{i}, d g_{i}\right) \rightarrow\left(g_{\infty}, d g_{\infty}\right)$ on A. Then, $\left(f_{i}+g_{i}, d\left(f_{i}+g_{i}\right)\right) \rightarrow\left(f_{\infty}+g_{\infty}, d\left(f_{\infty}+g_{\infty}\right)\right)$ at a.e. $w_{\infty} \in A$, and $\left(f_{i} g_{i}, d\left(f_{i} g_{i}\right)\right) \rightarrow\left(f_{\infty} g_{\infty}, d\left(f_{\infty} g_{\infty}\right)\right)$ at a.e. $w_{\infty} \in A$.

Proof. By Theorem 4.1, there exists a Borel subset $\hat{A}$ of $A$ such that $v(A \backslash$ $\hat{A})=0$ and that $\left|d f_{i}\right|^{2},\left\langle d f_{i}, d g_{i}\right\rangle$ and $\left|d g_{i}\right|^{2}$ converge strongly to $\left|d f_{\infty}\right|^{2}$, $\left\langle d f_{\infty}, d g_{\infty}\right\rangle$ and $\left|d g_{\infty}\right|^{2}$ on $\hat{A}$, respectively. Since $\left|d\left(f_{i} g_{i}\right)\right|^{2}=f_{i}^{2}\left|d g_{i}\right|^{2}+$ $2 f_{i} g_{i}\left\langle d f_{i}, d g_{i}\right\rangle+g_{i}\left|d f_{i}\right|^{2}$, by Proposition $4.1,\left|d\left(f_{i} g_{i}\right)\right|^{2}$ converges strongly to $f_{\infty}^{2}\left|d g_{\infty}\right|^{2}+2 f_{\infty} g_{\infty}\left\langle d f_{\infty}, d g_{\infty}\right\rangle+g_{\infty}^{2}\left|d f_{\infty}\right|^{2}=\left|d\left(f_{\infty} g_{\infty}\right)\right|^{2}$ on $\hat{A}$. On the other hand, since $d\left(f_{i} g_{i}\right)=g_{i} d f_{i}+f_{i} d g_{i}$, by Proposition 4.4, $\left\langle d r_{z_{i}}, d\left(f_{i} g_{i}\right)\right\rangle$ converges weakly to $g_{\infty}\left\langle d r_{z_{\infty}}, d f_{\infty}\right\rangle+f_{\infty}\left\langle d r_{z_{\infty}}, d g_{\infty}\right\rangle=\left\langle d r_{z_{\infty}}, d\left(f_{\infty} g_{\infty}\right)\right\rangle$ on $\hat{A}$ for every $z_{i} \rightarrow z_{\infty}$. Therefore, we have $\left(f_{i} g_{i}, d\left(f_{i} g_{i}\right)\right) \rightarrow\left(f_{\infty} g_{\infty}, d\left(f_{\infty} g_{\infty}\right)\right)$ on $\hat{A}$. Similarly, we have $\left(f_{i}+g_{i}, d\left(f_{i}+g_{i}\right)\right) \rightarrow\left(f_{\infty}+g_{\infty}, d\left(f_{\infty}+g_{\infty}\right)\right)$ on $\hat{A}$.

Corollary 4.4. Let $k$ be a positive integer, $\left\{A_{i}\right\}_{1 \leq i \leq \infty}$ a sequence of Borel subsets $A_{i}$ of $\bar{B}_{R}\left(x_{i}\right),\left\{f_{i}^{l}, g_{i}^{l}\right\}_{1 \leq i \leq \infty, 1 \leq l \leq k}$ a collection of Lipschitz functions $f_{i}^{l}, g_{i}^{l}$ on $B_{R}\left(x_{i}\right)$ with $\sup _{i}\left(\mathbf{L i p} f_{i}^{l}+\mathbf{L} \overline{\mathbf{i p}} g_{i}^{l}\right)<\infty$, and $\left\{F_{i}\right\}_{1 \leq i \leq \infty}$ a sequence of continuous functions on $\mathbf{R}^{k}$. Assume that the following properties hold:

1. $F_{i}$ converges to $F_{\infty}$ with respect to the compact uniformly topology.

2. $1_{A_{i}}$ converges weakly to $1_{A_{\infty}}$ at a.e. $w_{\infty} \in B_{R}\left(x_{\infty}\right)$.

3. $d f_{i}^{l} \rightarrow d f_{\infty}^{l}$ and $d g_{i}^{l} \rightarrow d g_{\infty}^{l}$ at a.e. $w_{\infty} \in A_{\infty}$ for every $1 \leq l \leq k$. 
Then we have

$$
\begin{aligned}
& \lim _{i \rightarrow \infty} \int_{A_{i}} F_{i}\left(\left\langle d f_{i}^{1}, d g_{i}^{1}\right\rangle, \ldots,\left\langle d f_{i}^{k}, d g_{i}^{k}\right\rangle\right) d \underline{\mathrm{vol}} \\
& \quad=\int_{A_{\infty}} F_{\infty}\left(\left\langle d f_{\infty}^{1}, d g_{\infty}^{1}\right\rangle, \ldots,\left\langle d f_{\infty}^{k}, d g_{\infty}^{k}\right\rangle\right) d v .
\end{aligned}
$$

Proof. The assertion follows from Propositions 4.1, 4.5 and Theorem 4.1.

We shall end this subsection by giving several remarks:

Remark 4.3. By several arguments in Section 3, and the proof of Theorem 4.1, we can also show the following: assume that the following properties hold:

1. $L^{2}$-energy of $\left\{f_{i}\right\}_{i}$ are upper semicontinuous at every $\alpha \in B_{R}\left(x_{\infty}\right)$,

2. There exists a dense subset $A$ of $B_{R}\left(x_{\infty}\right)$ and a Borel subset $\hat{A}$ of $B_{R}\left(x_{\infty}\right)$ such that $v\left(B_{R}\left(x_{\infty}\right) \backslash \hat{A}\right)=0$ and that $\left\langle d r_{w_{i}}, d f_{i}\right\rangle$ converges weakly to $\left\langle d r_{w_{\infty}}, d f_{\infty}\right\rangle$ at every $\alpha \in \hat{A}$ for every $w_{\infty} \in A$ and every $w_{i} \rightarrow w_{\infty}$.

Then, $d f_{i} \rightarrow d f_{\infty}$ on $B_{R}\left(x_{\infty}\right)$.

Remark 4.4. Let $\left\{\left(Y_{i}, y_{i}, v_{i}\right)\right\}_{1 \leq i \leq \infty}$ be a sequence of Ricci limit spaces and $\left\{f_{i}\right\}_{1 \leq i \leq \infty}$ a sequence of Lipschitz functions $f_{i}$ on $B_{R}\left(y_{i}\right)$. Then, similarly, we can also define a notion of convergence: $d f_{i} \rightarrow d f_{\infty}$ and give several properties as above.

Remark 4.5. Let $(Y, y, v)$ be a Ricci limit space and $\left\{f_{i}\right\}_{1 \leq i \leq \infty}$ a sequence of Lipschitz functions on $B_{R}(y)$ with $\sup _{i} \operatorname{Lip} f_{i}<\infty$. Then, $d f_{i} \rightarrow d f_{\infty}$ on $B_{R}(y)$ (in the sense of Definition 4.4 with respect to the convergence $\left.(Y, y, v) \stackrel{\left(\operatorname{id}_{Y}, R_{i}, \epsilon_{i}\right)}{\longrightarrow}(Y, y, v)\right)$ if and only if $\left|\operatorname{Lip}\left(f_{i}-f_{\infty}\right)\right|_{L^{2}\left(B_{R}(y)\right)} \rightarrow 0$. We shall check it below. By Corollary 4.4, it suffices to check "if" part. Assume that $\left|\operatorname{Lip}\left(f_{i}-f_{\infty}\right)\right|_{L^{2}\left(B_{R}(y)\right)} \rightarrow 0$. Then, it is clear that $L^{2}$-energy of $\left\{f_{i}\right\}_{i}$ are upper semicontinuous at every $w \in B_{R}(y)$. On the other hand, by Proposition 4.8, we have $\lim _{i \rightarrow \infty}\left|\operatorname{Lip}\left(r_{x_{i}}-r_{x_{\infty}}\right)\right|_{L^{2}\left(B_{R}(y)\right)}=0$ for every $x_{i} \rightarrow x_{\infty} \in$ $Y$. Especially, $\left\langle d r_{x_{i}}, d f_{i}\right\rangle$ converges weakly to $\left\langle d r_{x_{\infty}}, d f_{\infty}\right\rangle$ at every $w \in B_{R}(y)$. Thus, $d f_{i} \rightarrow d f_{\infty}$ on $B_{R}(y)$. 


\subsection{An approximation theorem}

Throughout this subsection, we shall use the following notation (same to one used in previous subsection): Let $\left\{\left(M_{i}, m_{i}\right)\right\}_{i}$ be a sequence of pointed $n$ dimensional complete Riemannian manifolds with $\operatorname{Ric}_{M_{i}} \geq-(n-1),(Y, y, v)$ a Ricci limit space of $\left\{\left(M_{i}, m_{i}, \underline{\mathrm{vol}}\right)\right\}_{i}, x_{i}$ a point in $M_{i}$ for every $i<\infty, x_{\infty}$ a point in $Y$ satisfying $\left(M_{i}, m_{i}, x_{i}, \underline{\mathrm{vol}}\right) \stackrel{\left(\phi_{i}, R_{i}, \epsilon_{i}\right)}{\longrightarrow}\left(Y, y, x_{\infty}, v\right)$. A purpose in this subsection is to give the following approximation theorem. Roughly speaking, it means that for a given Lipschitz function $f_{\infty}$ on $B_{R}\left(x_{\infty}\right)$, there exists a sequence of Lipschitz functions $f_{i}$ on $B_{R}\left(x_{i}\right)$ approximating the given function with respect to the topology: " $\left(f_{i}, d f_{i}\right) \rightarrow\left(f_{\infty}, d f_{\infty}\right)$ ".

Theorem 4.2 (Approximation theorem). Let $L, R$ be positive numbers, $f_{\infty}$ an L-Lipschitz function on $\bar{B}_{R}\left(x_{\infty}\right), A_{\infty}$ a compact subset of $\bar{B}_{R}\left(x_{\infty}\right)$, $\left\{A_{i}\right\}_{1 \leq i<\infty} \quad a$ sequence of Borel subsets $A_{i}$ of $\bar{B}_{R}\left(x_{i}\right)$, and $\left\{f_{i}\right\}_{1 \leq i<\infty}$ a sequence of L-Lipschitz functions $f_{i}$ on $A_{i}$. Assume that $\limsup \sup _{i \rightarrow \infty}^{G H} A_{i} \subset A_{\infty}$ and that $\left.f_{\infty}\right|_{A_{\infty}}$ is an extension of $\left\{f_{i}\right\}_{i}$ asymptotically. Then, for every $\epsilon>0$, there exist an open subset $\Omega_{\epsilon}$ of $B_{R}\left(x_{\infty}\right) \backslash A_{\infty}$, and a sequence $\left\{f_{i}^{\epsilon}\right\}_{1 \leq i \leq \infty}$ of $C(n, L)$-Lipschitz functions $f_{i}^{\epsilon}$ on $B_{R}\left(x_{i}\right)$ such that $\left(f_{i}^{\epsilon}, d f_{i}^{\epsilon}\right) \rightarrow\left(f_{\infty}^{\epsilon}, d f_{\infty}^{\epsilon}\right)$ on $\Omega_{\epsilon},\left.f_{i}^{\epsilon}\right|_{A_{i}}=\left.f_{i}\right|_{A_{i}}$ for every $1 \leq i \leq \infty$, and

$$
\begin{aligned}
& \frac{v\left(B_{R}\left(x_{\infty}\right) \backslash\left(\Omega_{\epsilon} \cup A_{\infty}\right)\right)}{v\left(B_{R}\left(x_{\infty}\right)\right)} \\
& \quad+\left|f_{\infty}-f_{\infty}^{\epsilon}\right|_{L^{\infty}\left(B_{R}\left(x_{\infty}\right)\right)}+\left|\operatorname{Lip}\left(f_{\infty}^{\epsilon}-f_{\infty}\right)\right|_{L^{2}\left(B_{R}\left(x_{\infty}\right)\right)}<\epsilon .
\end{aligned}
$$

Proof. Fix sufficiently small $\epsilon>0$ and $\xi>0$ (we will decide $\xi$ later). By Lemma 3.5 and (the proof of) Theorem 3.1, there exist collections of pairwise disjoint Borel subsets $\left\{E_{j}\right\}_{j}$ of $B_{R}\left(x_{\infty}\right)$, of positive numbers $\left\{\tau_{j}\right\}_{j}$, of positive integers $\left\{k_{j}\right\}_{j}$ with $1 \leq k_{j} \leq n$, and of points $\left\{x_{l}^{j}\right\}_{j, 1 \leq l \leq k_{j}}$ in $Y$ such that the following properties hold:

1. $v_{\infty}\left(B_{R}\left(x_{\infty}\right) \backslash \bigcup_{j} E_{j}\right)=0$ and $E_{j} \subset \bigcap_{l=1}^{k_{j}}\left(\mathcal{D}_{x_{l}^{j}}^{\tau_{j}} \backslash B_{\tau_{j}}\left(x_{l}^{j}\right)\right)$ for every $j$.

2. For every $w \in E_{j}$, we have

$$
\left\langle d r_{x_{l}^{j}}, d r_{x_{\hat{\imath}}^{j}}\right\rangle(w)=\lim _{r \rightarrow 0} \frac{1}{v\left(B_{r}(w)\right)} \int_{B_{r}(w)}\left\langle d r_{x_{l}^{j}}, d r_{x_{\hat{\imath}}^{j}}\right\rangle d v=\delta_{l, \hat{l}} \pm \epsilon .
$$


3. For every $w \in E_{j}$, there exists $r_{w}>0$ such that $r_{w} \ll \tau_{j}, \bar{B}_{10 r_{w}}(w) \subset$ $\mathrm{B}_{R}\left(x_{\infty}\right)$ and

$$
\frac{1}{v\left(B_{t}(w)\right)} \int_{B_{t}(w)}\left|d f_{\infty}-d\left(\sum_{l=1}^{k_{j}} a_{l}^{j}(w) r_{x_{l}^{j}}\right)\right|^{2} d v<\epsilon
$$

for every $0<t<r_{w}$.

Put $X=\bigcup_{j=1}^{\infty}\left(E_{j} \backslash \overline{\mathrm{B}}_{5 \xi}\left(A_{\infty}\right)\right)$. By Proposition 2.2, there exists a pairwise disjoint collection $\left\{\bar{B}_{r_{i}}\left(z_{i}\right)\right\}_{i} \subset B_{R}\left(x_{\infty}\right)$ such that $z_{i} \in X, r_{i}<<\min \left\{r_{z_{i}}, \epsilon, \xi\right\}$ and $X \backslash \bigcup_{i=1}^{N} \bar{B}_{r_{i}}\left(z_{i}\right) \subset \bigcup_{i=N+1}^{\infty} \bar{B}_{5 r_{i}}\left(z_{i}\right)$ for every $N$. For every $i$, let $l(i)$ with $z_{i} \in E_{l(i)}$. Without loss of generality, we can assume that $l(i)=i$. Fix $N$ satisfying $\sum_{i=N+1}^{\infty} v\left(B_{r_{i}}\left(z_{i}\right)\right)<\epsilon$. Let $z_{i}(j) \rightarrow z_{i}$ and $x_{m}^{l}(j) \rightarrow x_{m}^{l}$. Define functions $F_{i}^{j}$ on $B_{r_{i}}\left(z_{i}(j)\right)$, and $F_{i}$ on $B_{r_{i}}\left(z_{i}\right)$ by

$$
F_{i}^{j}=\sum_{m=1}^{k_{i}} a_{m}^{i} r_{x_{m}^{i}(j)}+C_{i}, F_{i}=\sum_{m=1}^{k_{i}} a_{m}^{i} r_{x_{m}^{i}}+C_{i}
$$

where $C_{i}$ is the constant defined by satisfying $F_{i}\left(z_{i}\right)=f_{\infty}\left(z_{i}\right)$, and $a_{m}^{i}=$ $a_{m}^{i}\left(z_{i}\right)$.

Claim 4.1. We have $\mathbf{L i p} F_{i}^{j}+\mathbf{L i p} F_{i} \leq C(n, L)$ for every $i, j$.

The proof is as follows. Since

$$
\begin{aligned}
\left|d f_{\infty}\left(z_{i}\right)\right|^{2} & =\sum_{s, t} a_{s}^{i} a_{t}^{i}\left\langle d r_{x_{s}^{i}}, d r_{x_{t}^{i}}\right\rangle\left(z_{i}\right) \\
& =\sum_{s, t} a_{s}^{i} a_{t}^{i}\left(\delta_{s, t} \pm \epsilon\right) \\
& =(1 \pm \epsilon) \sum_{s=1}^{k_{i}}\left(a_{s}^{i}\right)^{2} \pm \Psi(\epsilon ; n) \sum_{s=1}^{k_{i}}\left(a_{s}^{i}\right)^{2}=(1 \pm \Psi(\epsilon ; n)) \sum_{s=1}^{k_{i}}\left(a_{s}^{i}\right)^{2}
\end{aligned}
$$

and $\left|d f_{\infty}\right|\left(z_{i}\right) \leq L$, we have $\sum_{m=1}^{k_{i}}\left(a_{m}^{i}\right)^{2} \leq L^{2}+\Psi(\epsilon ; n, L)$. Therefore, we have Claim 4.1.

We remark that $\left\{\bar{B}_{r_{i}}\left(z_{i}(j)\right)\right\}_{1 \leq i \leq N}$ is a pairwise disjoint collection for every sufficiently large $j$. Define functions $F_{j}$ on $\bigcup_{m=1}^{N} \bar{B}_{(1-\xi) r_{i}}\left(z_{i}(j)\right)$, and $F_{\infty}$ on $\bigcup_{m=1}^{N} \bar{B}_{(1-\xi) r_{i}}\left(z_{i}\right)$ by $\left.F_{j}\right|_{B_{(1-\xi) r_{i}}\left(z_{i}(j)\right)}=\left.F_{j}^{i}\right|_{B_{(1-\xi) r_{i}}\left(z_{i}(j)\right)},\left.F_{\infty}\right|_{B_{(1-\xi) r_{i}}\left(z_{i}\right)}=$ $\left.F_{j}\right|_{B_{(1-\xi) r_{i}}\left(z_{i}\right)}$ for every sufficiently large $j$. 
Claim 4.2. We have $\mathbf{L i p} F_{j}+\mathbf{L i p} F_{\infty} \leq C(n, L)+\xi^{-1} \Psi(\epsilon ; n, L)$ for every sufficiently large $j$.

The proof is as follows. By Claim 4.1, we have $\operatorname{Lip}\left(\left.F_{j}\right|_{\bar{B}_{(1-\xi) r_{i}}\left(z_{i}(j)\right)}\right)+$ $\operatorname{Lip}\left(\left.F_{\infty}\right|_{\bar{B}_{(1-\xi) r_{i}}\left(z_{i}\right)}\right) \leq C(n, L)$ for every $i, j$. Let $j_{0}$ satisfying that $\epsilon_{j}<<$ $\min \left\{\xi r_{1}, \ldots, \xi r_{N}\right\}$ for every $j \geq j_{0}$. Fix $j \geq j_{0}, 1 \leq l<m \leq N, w_{l}(j) \in$ $\bar{B}_{(1-\xi) r_{l}}\left(z_{l}(j)\right) \quad$ and $\quad w_{m}(j) \in \bar{B}_{(1-\xi) r_{m}}\left(z_{m}(j)\right)$. Since $\bar{B}_{r_{l}}\left(z_{l}(j)\right) \cap \bar{B}_{r_{m}}$ $\left(z_{m}(j)\right)=\emptyset$, there exists $\alpha(j) \in \partial B_{r_{l}}\left(z_{l}\right)$ such that $\overline{w_{l}(j), \alpha(j)}+\overline{\alpha(j), w_{m}(j)}$ $=\overline{w_{l}(j), w_{m}(j)}$. Thus, we have $\overline{w_{l}(j), w_{m}(j)} \geq \overline{w_{l}(j), \alpha(j)} \geq \xi r_{l}$. Similarly, we have $\overline{w_{l}(j), w_{m}(j)} \geq \xi r_{m}$. Thus, we have $\overline{w_{l}(j), w_{m}(j)} \geq \xi\left(r_{l}+r_{m}\right) / 2$. On the other hand, since

$$
\frac{1}{v\left(B_{10 r_{l}}\left(z_{l}\right)\right)} \int_{B_{10 r_{l}}\left(z_{l}\right)}\left|\operatorname{Lip}\left(f_{\infty}-\sum_{s=1}^{k_{l}} a_{s}^{l} r_{x_{s}^{l}}\right)\right|^{2} d v<\epsilon
$$

by the segment inequality on limit spaces [6, Theorem 2.6], there exist points $\hat{z}_{l}, \phi_{j}\left(\hat{w_{l}}(j)\right)$ in $B_{r_{l}}\left(z_{l}\right)$ and a minimal geodesic $\gamma$ from $\hat{z}_{l}$ to $\phi_{j}\left(\hat{w_{l}}(j)\right)$ such

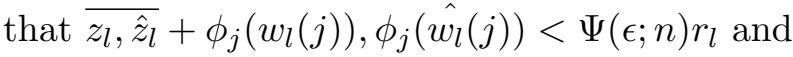

$$
\int_{0}^{\overline{z_{l}, \phi_{j}\left(\hat{w_{l}}(j)\right)}} \operatorname{Lip}\left(f_{\infty}-\sum_{s=1}^{k_{l}} a_{s}^{l} r_{x_{s}^{l}}\right)(\gamma(t)) d t<\Psi(\epsilon ; n) r_{l} .
$$

Therefore, we have

$$
\begin{aligned}
& \left|f_{\infty}\left(\hat{z}_{l}\right)-\sum_{s=1}^{k_{l}} a_{s}^{l} r_{x_{s}^{l}}\left(\hat{z}_{l}\right)-\left(f_{\infty}\left(\phi_{j}\left(\hat{z_{l}}(j)\right)\right)-\sum_{s=1}^{k_{l}} a_{s}^{l} r_{x_{s}^{l}}\left(\phi_{j}\left(\hat{z_{l}}(j)\right)\right)\right)\right| \\
& \leq \int_{0}^{\hat{\hat{z}_{l}, \phi_{j}\left(\hat{w}_{l}(j)\right)}} \operatorname{Lip}\left(f_{\infty}-\sum_{s=1}^{k_{l}} a_{s}^{l} r_{x_{s}^{l}}\right)(\gamma(t)) d t<\Psi(\epsilon ; n) r_{l} .
\end{aligned}
$$

Thus, we have

$$
\begin{aligned}
& \left|f_{\infty}\left(z_{l}\right)-\sum_{s=1}^{k_{l}} a_{s}^{l} r_{x_{s}^{l}}\left(z_{l}\right)-\left(f_{\infty}\left(\phi_{j}\left(z_{l}(j)\right)\right)-\sum_{s=1}^{k_{l}} a_{s}^{l} r_{x_{s}^{l}}\left(\phi_{j}\left(z_{l}(j)\right)\right)\right)\right| \\
& \quad \leq \Psi(\epsilon ; n, L) r_{l} .
\end{aligned}
$$

Especially, we have $\left|F_{j}\left(w_{l}(j)\right)-f_{\infty} \circ \phi_{j}\left(w_{l}(j)\right)\right| \leq \Psi(\epsilon ; n, L) r_{l}$. Similarly, we have $\left|F_{j}\left(w_{m}(j)\right)-f_{\infty} \circ \phi_{j}\left(w_{m}(j)\right)\right| \leq \Psi(\epsilon ; n, L) r_{m} \quad$ and $\quad\left|F_{\infty}-f_{\infty}\right| \leq \Psi$ 
$(\epsilon ; n, L) r_{l}$ on $\bar{B}_{(1-\xi) r_{l}}\left(z_{l}\right)$. Therefore, we have

$$
\begin{aligned}
\left|F_{j}\left(w_{l}(j)\right)-F_{j}\left(w_{m}(j)\right)\right| \leq & \left|f_{\infty} \circ \phi_{j}\left(w_{l}(j)\right)-f_{\infty} \circ \phi_{j}\left(w_{l}(j)\right)\right| \\
& +\Psi(\epsilon ; n, L)\left(r_{l}+r_{m}\right) \\
\leq & L \overline{\phi_{j}\left(w_{l}(j)\right), \phi_{j}\left(w_{m}(j)\right)}+\Psi(\epsilon ; n, L)\left(r_{l}+r_{m}\right) \\
\leq & \left.L \overline{\left(w_{l}(j), w_{m}(j)\right.}+\epsilon_{j}\right)+\Psi(\epsilon ; n, L)\left(r_{l}+r_{m}\right) \\
\leq & L \overline{w_{l}(j), w_{m}(j)}+\Psi(\epsilon ; n, L)\left(r_{l}+r_{m}\right) \\
\leq & \left(L+\xi^{-1} \Psi(\epsilon ; n, L)\right) \overline{w_{l}(j), w_{m}(j)} .
\end{aligned}
$$

Thus, by Claim 4.1, we have $\operatorname{Lip} F_{j} \leq C(n, L)+\xi^{-1} \Psi(\epsilon ; n, L)$. Similarly, we have $\operatorname{Lip} F_{\infty} \leq C(n, L)+\xi^{-1} \Psi(\epsilon ; n, L)$. Therefore, we have Claim 4.2.

Claim 4.3. We have $\bigcup_{i=1}^{N} \bar{B}_{(1-\xi) r_{i}}\left(z_{i}(j)\right) \subset M_{i} \backslash B_{2 \xi}\left(A_{i}\right)$ and $\bigcup_{i=1}^{N}$ $\bar{B}_{(1-\xi) r_{i}}\left(z_{i}\right) \subset Y \backslash B_{2 \xi}\left(A_{\infty}\right)$ for every sufficiently large $j$.

The proof is as follows. It is easy to check that $\bigcup_{i=1}^{N} \bar{B}_{r_{i}}\left(z_{i}\right) \subset Y \backslash$ $B_{2 \xi}\left(A_{\infty}\right)$. On the other hand, by the assumption, there exists $i_{0}$ such that $\phi_{i}\left(A_{i}\right) \subset B_{\xi}\left(A_{\infty}\right)$ and $\epsilon_{i}<<\min _{1 \leq j \leq N}\left\{\xi r_{j}\right\}$ for every $i \geq i_{0}$. Thus, since $\phi_{i}\left(\bigcup_{i=1}^{N} \bar{B}_{(1-\xi) r_{i}}\left(z_{i}(j)\right)\right) \subset \bigcup_{i=1}^{N} \bar{B}_{r_{i}}\left(z_{i}\right) \subset Y \backslash B_{4 \xi}\left(A_{\infty}\right)$ for every $i \geq i_{0}$, we have Claim 4.3.

Claim 4.4. We have

$$
\lim _{i \rightarrow \infty} \sup _{A_{i}}\left|f_{i}-f_{\infty} \circ \phi_{i}\right|=0
$$

The proof is done by a contradiction. Assume that the assertion is false. Then, there exists $\tau>0$, a subsequence $\{n(i)\}_{i}$ of $\mathbf{N}$, and $\alpha_{n(i)} \in A_{n(i)}$ such that $\left|f_{n(i)}\left(\alpha_{n(i)}\right)-f_{\infty} \circ \phi_{n(i)}\left(\alpha_{n(i)}\right)\right|>\tau$. Without loss of generality, we can assume that there exists $\alpha_{\infty} \in Y$ such that $\phi_{n(i)}\left(\alpha_{n(i)}\right) \rightarrow \alpha_{\infty}$. Thus, $\liminf _{i \rightarrow \infty}\left|f_{n(i)}\left(\alpha_{n(i)}\right)-f_{\infty}\left(\alpha_{\infty}\right)\right| \geq \tau$. On the other hand, we have $\alpha_{\infty} \in$ $\overline{A_{\infty}}=A_{\infty}$. Since $\left.f_{\infty}\right|_{A_{\infty}}$ is an extension of $\left\{f_{i}\right\}_{i}$ asymptotically, this is a cotradiction. Therefore, we have Claim 4.4.

Put $W_{j}=\bigcup_{m=1}^{N} B_{(1-\xi) r_{i}}\left(z_{i}(j)\right)$ and $W_{\infty}=\bigcup_{m=1}^{N} B_{(1-\xi) r_{i}}\left(z_{i}\right)$. By Claim 4.3, we can define Lipschitz functions $G_{j}$ on $W_{j} \cup A_{j}$, and $G_{\infty}$ on $W_{\infty} \cup A_{\infty}$ by $\left.G_{j}\right|_{W_{j}}=\left.F_{j}\right|_{W_{j}},\left.G_{j}\right|_{A_{j}}=f_{j},\left.G_{\infty}\right|_{W_{\infty}}=\left.F_{\infty}\right|_{W_{\infty}}$ and $\left.G_{\infty}\right|_{A_{\infty}}=\left.f_{\infty}\right|_{A_{\infty}}$ for every sufficiently large $j$.

Claim 4.5. We have $\operatorname{Lip} G_{j}+\operatorname{Lip} G_{\infty} \leq C(n, L)+\xi^{-1} \Psi(\epsilon ; n, L)$ for every sufficiently large $j$. 
The proof is as follows. Put $\xi_{j}=\sup _{A_{j}}\left|f_{j}-f_{\infty} \circ \phi_{j}\right|$. Then by the proof of Claim 4.2, there exists $j_{0}$ such that

$$
\begin{aligned}
\left|G_{j}\left(\alpha_{j}\right)-G_{j}\left(\beta_{j}\right)\right| & =\left|F_{j}\left(\alpha_{j}\right)-f_{j}\left(\beta_{j}\right)\right| \\
& \leq\left|F_{\infty} \circ \phi_{j}\left(\alpha_{j}\right)-f_{\infty} \circ \phi_{j}\left(\beta_{j}\right)\right|+\Psi(\epsilon ; n, L) r_{i}+\xi_{j} \\
& \leq\left|f_{\infty} \circ \phi_{j}\left(\alpha_{j}\right)-f_{\infty} \circ \phi_{j}\left(\beta_{j}\right)\right|+\Psi(\epsilon ; n, L) r_{i}+\xi_{j} \\
& \leq L \overline{\phi_{j}\left(\alpha_{j}\right), \phi_{j}\left(\beta_{j}\right)}+\Psi(\epsilon ; n, L) r_{i} \\
& \leq L\left(\overline{\alpha_{j}, \beta_{j}}+\epsilon_{j}\right)+\Psi(\epsilon ; n, L) \xi \leq(L+\Psi(\epsilon ; n, L)) \overline{\alpha_{j}, \beta_{j}}
\end{aligned}
$$

for every $j \geq j_{0}$, every $\alpha_{j} \in \bar{B}_{(1-\xi) r_{i}}\left(z_{i}(j)\right)$ and every $\beta_{j} \in A_{j}$. Therefore, by Claim 4.2, we have $\operatorname{Lip} G_{j} \leq C(n, L)+\xi^{-1} \Psi(\epsilon ; n, L)$ for every sufficiently large $j$. Similarly, we have $\mathbf{L i p} G_{\infty} \leq C(n, L)+\xi^{-1} \Psi(\epsilon ; n, L)$. Thus, we have Claim 4.5.

For $\Psi=\Psi(\epsilon ; n, L)$ as in Claim 4.5, put $\xi=\sqrt{\Psi}$. Let $f_{j}^{\epsilon}$ be a Lipschitz function on $M_{j}$ and $f_{\infty}^{\epsilon}$ a Lipschitz function on $Y$ satisfying that $\operatorname{Lip} f_{j}^{\epsilon}=\operatorname{Lip} G_{j}, \operatorname{Lip} f_{\infty}^{\epsilon}=\operatorname{Lip} G_{\infty},\left.f_{j}^{\epsilon}\right|_{W_{j} \cup A_{j}}=\left.F_{j}\right|_{W_{j} \cup A_{j}}$ and $\left.f_{\infty}^{\epsilon}\right|_{W_{\infty} \cup A_{\infty}}=$ $\left.F_{\infty}\right|_{W_{\infty} \cup A_{\infty}}$. Put $\Omega_{\epsilon}=W_{\infty}$. Then, by Proposition 4.8 and Corollary 4.3, we have $\left(f_{i}^{\epsilon}, d f_{i}^{\epsilon}\right) \rightarrow\left(f_{\infty}^{\epsilon}, d f_{\infty}^{\epsilon}\right)$ on $\Omega_{\epsilon}$. On the other hand, we have $v\left(B_{R}\left(x_{\infty}\right) \backslash\right.$ $\left.\left(\Omega_{\epsilon} \cup A_{\infty}\right)\right) \leq v\left(X \backslash \Omega_{\epsilon}\right)+v\left(\bar{B}_{5 \xi}\left(A_{\infty}\right) \backslash A_{\infty}\right) \leq \sum_{i=N+1}^{\infty} v\left(B_{5 r_{i}}\left(z_{i}\right)\right)+v$ $\left(\bar{B}_{5 \xi}\left(A_{\infty}\right) \backslash A_{\infty}\right)+\Psi(\epsilon ; n, L) \leq C(n) \epsilon+v\left(\bar{B}_{5 \xi}\left(A_{\infty}\right) \backslash A_{\infty}\right)+\Psi(\epsilon ; n, L)$ and

$$
\begin{aligned}
\int_{B_{R}\left(x_{\infty}\right)}\left|d f_{\infty}-d f_{\infty}^{\epsilon}\right|^{2} d v \leq & \int_{X}\left|d f_{\infty}-d f_{\infty}^{\epsilon}\right|^{2} d v+\int_{\bar{B}_{5 \xi}\left(A_{\infty}\right)}\left|d f_{\infty}-d f_{\infty}^{\epsilon}\right|^{2} d v \\
\leq & \sum_{i=1}^{N} \int_{B_{(1-\xi) r_{i}}\left(z_{i}\right)}\left|d f_{\infty}-d f_{\infty}^{\epsilon}\right|^{2} d v \\
& +5 L^{2} v\left(B_{5 \xi}\left(A_{\infty}\right) \backslash A_{\infty}\right)+\int_{A_{\infty}}\left|d f_{\infty}^{\epsilon}-d f_{\infty}\right|^{2} d v \\
& +\Psi(\epsilon ; n, L) \\
\leq & \sum_{i=1}^{N} \epsilon v\left(B_{(1-\xi) r_{i}}\left(z_{i}\right)\right)+5 L^{2} v\left(B_{5 \xi}\left(A_{\infty}\right) \backslash A_{\infty}\right) \\
& +\Psi(\epsilon ; n, L) \\
\leq & \epsilon v\left(B_{R}\left(x_{\infty}\right)\right)+5 L^{2} v\left(B_{5 \xi}\left(A_{\infty}\right) \backslash A_{\infty}\right) \\
& +\Psi(\epsilon ; n, L) .
\end{aligned}
$$

We remark that since $A_{\infty}$ is compact, we have $\lim _{r \rightarrow 0} v\left(B_{r}\left(A_{\infty}\right) \backslash A_{\infty}\right)=0$. Put $\tau(r)=v\left(B_{r}\left(A_{\infty}\right) \backslash A_{\infty}\right)$. On the other hand, by the proof of Claim 4.2, we have $\left|f_{\infty}^{\epsilon}-f_{\infty}\right|<\Psi(\epsilon ; n, L)$ on $\Omega_{\epsilon} \cup A_{\infty}$. For every $w \in B_{R}\left(x_{\infty}\right)$, there 
exists $\hat{w} \in \Omega_{\epsilon} \cup A_{\infty}$ such that $\overline{w, \hat{w}}<\Psi\left(\epsilon, \tau(5 \xi) ; n, L, v\left(B_{R}\left(x_{\infty}\right)\right)\right)$. Therefore, we have $\left|f_{\infty}^{\epsilon}(w)-f_{\infty}(w)\right| \leq\left|f_{\infty}^{\epsilon}(\hat{w})-f_{\infty}(\hat{w})\right|+\Psi\left(\epsilon, \tau(5 \xi) ; n, L, v\left(B_{R}\right.\right.$ $\left.\left.\left(x_{\infty}\right)\right)\right) \leq \Psi\left(\epsilon, \tau(5 \xi) ; n, L, v\left(B_{R}\left(x_{\infty}\right)\right)\right)$. Thus, we have $\left|f_{\infty}^{\epsilon}-f_{\infty}\right|<\Psi(\epsilon, \tau$ $\left.(5 \xi) ; n, L, v\left(B_{R}\left(x_{\infty}\right)\right)\right)$ on $B_{R}\left(x_{\infty}\right)$. Since it is not difficult to check that $\left|\operatorname{Lip}\left(f_{\infty}^{\epsilon}-f_{\infty}\right)\right|_{L^{2}\left(B_{R}\left(x_{\infty}\right)\right)} \leq \Psi\left(\epsilon ; n, L, R, v\left(B_{R}\left(x_{\infty}\right)\right)\right)$, we have the assertion.

By using Theorem 4.2, we shall give a sufficient condition to satisfy pointwise upper semicontinuity of $L^{2}$-energy:

Proposition 4.9. Let $R$ be a positive number, $f_{i}$ a $C^{2}$-function on $B_{R}\left(x_{i}\right)$ for every $i<\infty$, and $f_{\infty}$ a Lipschitz function on $\bar{B}_{R}\left(x_{\infty}\right)$. Assume that

$$
\sup _{i}\left(\operatorname{Lip} f_{i}+\int_{B_{R}\left(x_{i}\right)}\left|\Delta f_{i}\right| d \underline{\mathrm{vol}}\right)<\infty
$$

and $f_{i} \rightarrow f_{\infty}$ on $B_{R}\left(x_{\infty}\right)$. Then, we have

$$
\limsup _{i \rightarrow \infty} \int_{B_{R}\left(x_{i}\right)}\left(\operatorname{Lip} f_{i}\right)^{2} d \underline{\mathrm{vol}} \leq \int_{B_{R}\left(x_{\infty}\right)}\left(\operatorname{Lip} f_{\infty}\right)^{2} d v .
$$

Especially, $L^{2}$-energy of $\left\{f_{i}\right\}_{i}$ are upper semicontinuous at every $w \in B_{R}\left(x_{\infty}\right)$.

Proof. Let $g_{i}=\Delta f_{i}$. First, we shall remark the following:

Claim 4.6. We have

$$
\begin{gathered}
\int_{B_{R}\left(x_{i}\right)}\left|d\left(f_{i}+k\right)\right|^{2} d \underline{\mathrm{vol}}-2 \int_{B_{R}\left(x_{i}\right)} g_{i}\left(f_{i}+k\right) d \underline{\mathrm{vol}} \\
\geq \int_{B_{R}\left(x_{i}\right)}\left|d f_{i}\right|^{2} d \underline{\mathrm{vol}}-2 \int_{B_{R}\left(x_{i}\right)} g_{i} f_{i} d \underline{\mathrm{vol}}
\end{gathered}
$$

for every Lipschitz function $k$ on $B_{R}\left(x_{i}\right)$, which has compact support.

Claim 4.6 follows from the equality:

$$
\begin{aligned}
& \int_{B_{R}\left(x_{i}\right)}\left|d\left(f_{i}+k\right)\right|^{2} d \underline{\mathrm{vol}}-2 \int_{B_{R}\left(x_{i}\right)} g_{i}\left(f_{i}+k\right) d \underline{\mathrm{vol}} \\
& =\int_{B_{R}\left(x_{i}\right)}\left|d f_{i}\right|^{2} d \underline{\mathrm{vol}}-2 \int_{B_{R}\left(x_{i}\right)} g_{i} f_{i} d \underline{\mathrm{vol}} \\
& \quad+\int_{B_{R}\left(x_{i}\right)}|d k|^{2} d \underline{\mathrm{vol}} .
\end{aligned}
$$


Fx $\epsilon>0$. Let $L \geq 1$ with

$$
\sup _{i}\left(\operatorname{Lip} f_{i}+\left|f_{i}\right|_{L^{\infty}\left(B_{R}\left(x_{i}\right)\right)}+\int_{B_{R}\left(x_{i}\right)}\left|g_{i}\right| d \underline{\mathrm{vol}}\right)<L .
$$

Since $\lim \sup _{i \rightarrow \infty}^{\mathrm{GH}} A_{R-\epsilon, R}\left(x_{i}\right) \subset A_{R-\epsilon, R}\left(x_{\infty}\right)$, by Theorem 4.2 , there exists a sequence $\left\{f_{i}^{\epsilon}\right\}_{1 \leq i \leq \infty}$ of $C(n, L)$-Lipschitz functions $f_{i}^{\epsilon}$ on $B_{R}\left(x_{i}\right)$, and an open set $\Omega_{\epsilon} \subset B_{R}\left(x_{\infty}\right) \backslash A_{R-\epsilon, R}\left(x_{\infty}\right)$ such that $\left.f_{i}^{\epsilon}\right|_{A_{R-\epsilon, R}\left(x_{i}\right)}=\left.f_{i}\right|_{A_{R-\epsilon, R}\left(x_{i}\right)}$ for every $1 \leq i \leq \infty,\left(f_{i}^{\epsilon}, d f_{i}^{\epsilon}\right) \rightarrow\left(f_{\infty}^{\epsilon}, d f_{\infty}^{\epsilon}\right)$ on $\Omega_{\epsilon}$, and

$$
\begin{aligned}
& \frac{v\left(B_{R}\left(x_{\infty}\right) \backslash\left(\Omega_{\epsilon} \cup A_{R-\epsilon, R}\left(x_{\infty}\right)\right)\right)}{v\left(B_{R}\left(x_{\infty}\right)\right)}+\left|f_{\infty}-f_{\infty}^{\epsilon}\right|_{L^{\infty}\left(B_{R}\left(x_{\infty}\right)\right)} \\
& \quad+\left|\operatorname{Lip}\left(f_{\infty}^{\epsilon}-f_{\infty}\right)\right|_{L^{2}\left(B_{R}\left(x_{\infty}\right)\right)}<\epsilon .
\end{aligned}
$$

By Claim 4.6, we have

$\int_{B_{R}\left(x_{i}\right)}\left|d f_{i}^{\epsilon}\right|^{2} d \underline{\mathrm{vol}}-2 \int_{B_{R}\left(x_{i}\right)} g_{i} f_{i}^{\epsilon} d \underline{\mathrm{vol}} \geq \int_{B_{R}\left(x_{i}\right)}\left|d f_{i}\right|^{2} d \underline{\mathrm{vol}}-2 \int_{B_{R}\left(x_{i}\right)} g_{i} f_{i} d \underline{\mathrm{vol}}$.

By Proposition 2.2, without loss of generality, we can assume that there exists a pairwise disjoint finite collection $\left\{\bar{B}_{r_{i}}\left(z_{i}\right)\right\}_{1 \leq i \leq N}$ such that $\Omega_{\epsilon}=$ $\bigcup_{i=1}^{N} B_{r_{i}}\left(z_{i}\right)$. Let $z_{i}(j) \rightarrow z_{i}$. Put $\Omega_{\epsilon}(j)=\bigcup_{i=1}^{N} B_{r_{i}}\left(z_{i}(j)\right)$. Since $\underline{\operatorname{vol}}\left(\Omega_{\epsilon}(j) \cup\right.$ $\left.A_{R-\epsilon, R}\left(x_{j}\right)\right) / \underline{\text { vol }} B_{R}\left(x_{j}\right) \geq 1-\epsilon$ for every sufficiently large $j$, by Proposition 4.7 , we have

$$
\left.\left|\int_{B_{R}\left(x_{j}\right)}\right| d f_{j}^{\epsilon}\right|^{2} d \underline{\mathrm{vol}}-\int_{B_{R}\left(x_{\infty}\right)}\left|d f_{\infty}\right|^{2} d v \mid<\Psi(\epsilon ; n, L, R) .
$$

On the other hand, since $\sup _{B_{R}\left(x_{j}\right)}\left|f_{j}^{\epsilon}-f_{j}\right| \leq C(n, R, L) \sup _{\Omega_{\epsilon}(j)}\left|f_{j}^{\epsilon}-f_{j}\right|$ and $\lim \sup _{j \rightarrow \infty} \sup _{\Omega_{\epsilon}(j)}\left|f_{j}^{\epsilon}-f_{j}\right| \leq \sup _{\Omega_{\epsilon}}\left|f_{\infty}^{\epsilon}-f_{\infty}\right|$, we have

$$
\begin{aligned}
\left|\int_{B_{R}\left(x_{j}\right)} g_{j} f_{j}^{\epsilon} d \underline{\mathrm{vol}}-\int_{B_{R}\left(x_{j}\right)} g_{j} f_{j} d \underline{\mathrm{vol}}\right| & \leq \sup _{B_{R}\left(x_{j}\right)}\left|f_{j}^{\epsilon}-f_{j}\right| \int_{B_{R}\left(x_{j}\right)}\left|g_{j}\right| d \underline{\mathrm{vol}} \\
& \leq \Psi(\epsilon ; n, R, L)
\end{aligned}
$$

for every sufficiently large $j$. Therefore, we have

$$
\limsup _{i \rightarrow \infty} \int_{B_{R}\left(x_{i}\right)}\left|d f_{i}\right|^{2} d \underline{\mathrm{vol}} \leq \int_{B_{R}\left(x_{\infty}\right)}\left|d f_{\infty}\right|^{2} d v+\Psi(\epsilon ; n, L, R) .
$$

By letting $\epsilon \rightarrow 0$, we have the assertion. 
Next corollary follows from Remark 4.2 and Proposition 4.9 directly. See also $[16,35]$ :

Corollary 4.5. Let $R$ be a positive number, $f_{i}$ a $C^{2}$-function on $B_{R}\left(x_{i}\right)$ for every $i<\infty$, and $f_{\infty}$ a Lipschitz function on $B_{R}\left(x_{\infty}\right)$. Assume that

$$
\sup _{i}\left(\operatorname{Lip} f_{i}+\int_{B_{R}\left(x_{i}\right)}\left|\Delta f_{i}\right|^{2} d \underline{\mathrm{vol}}\right)<\infty
$$

and $f_{i} \rightarrow f_{\infty}$ on $B_{R}\left(x_{\infty}\right)$. Then, we have $\left(f_{i}, d f_{i}\right) \rightarrow\left(f_{\infty}, d f_{\infty}\right)$ on $B_{R}\left(x_{\infty}\right)$.

Next we shall consider a convergence of the equations $\Delta f_{i}=g_{i}$ with respect to the measured Gromov-Hausdorff convergence:

Corollary 4.6. Let $R$ be a positive number, $f_{i}$ a $C^{2}$-function on $B_{R}\left(x_{i}\right)$ for every $i<\infty$, and $f_{\infty}$ a Lipschitz function on $B_{R}\left(x_{\infty}\right)$ with $\sup _{i}\left(\operatorname{Lip} f_{i}+\right.$ $\left.\left|\Delta f_{i}\right|_{L^{\infty}\left(B_{R}\left(x_{i}\right)\right)}\right)<\infty$. Assume that $f_{i} \rightarrow f_{\infty}$ on $B_{R}\left(x_{\infty}\right)$ and that there exists a $L^{\infty}$-function $g_{\infty}$ on $B_{R}\left(x_{\infty}\right)$ such that $\Delta f_{i}$ converges weakly to $g_{\infty}$ at a.e. $w \in B_{R}\left(x_{\infty}\right)$. Then, we have

$$
\int_{B_{R}\left(x_{\infty}\right)}\left\langle d f_{\infty}, d k_{\infty}\right\rangle d v=\int_{B_{R}\left(x_{\infty}\right)} k_{\infty} g_{\infty} d v
$$

for every Lipschitz function $k_{\infty}$ on $B_{R}\left(x_{\infty}\right)$, which has compact support.

Proof. By Corollary 4.5, we have $\left(f_{i}, d f_{i}\right) \rightarrow\left(f_{\infty}, d f_{\infty}\right)$ on $B_{R}\left(x_{\infty}\right)$. Let $L \geq$ 1 with $\sup _{i}\left(\operatorname{Lip} f_{i}+\left|f_{i}\right|_{L^{\infty}\left(B_{R}\left(x_{i}\right)\right)}+\left|\Delta f_{i}\right|_{L^{\infty}\left(B_{R}\left(x_{i}\right)\right)}\right)<L$. Put $r=$ $\sup _{w \in \operatorname{supp} k_{\infty}} \overline{x_{\infty}, w}$ and $g_{i}=\Delta f_{i}$. Then, we have $r<R$. Fix $\epsilon>0$ with $\epsilon<R-r$. By Theorem 4.2, there exists a sequence $\left\{k_{i}^{\epsilon}\right\}_{1 \leq i \leq \infty}$ of $C(n, L)$ Lipschitz functions $k_{i}^{\epsilon}$ on $B_{R}\left(x_{i}\right)$, and an open set $\Omega_{\epsilon} \subset B_{R}\left(x_{\infty}\right) \backslash A_{R-\epsilon, R}\left(x_{\infty}\right)$ such that $\left.k_{i}^{\epsilon}\right|_{A_{R-\epsilon, R}\left(x_{i}\right)}=0$ for every $1 \leq i \leq \infty,\left(k_{i}^{\epsilon}, d k_{i}^{\epsilon}\right) \rightarrow\left(k_{\infty}^{\epsilon}, d k_{\infty}^{\epsilon}\right)$ on $\Omega_{\epsilon}$ and

$$
\begin{aligned}
& \frac{v\left(B_{R}\left(x_{\infty}\right) \backslash\left(\Omega_{\epsilon} \cup A_{R-\epsilon, R}\left(x_{\infty}\right)\right)\right)}{v\left(B_{R}\left(x_{\infty}\right)\right)}+\left|k_{\infty}-k_{\infty}^{\epsilon}\right|_{L^{\infty}\left(B_{R}\left(x_{\infty}\right)\right)} \\
& \quad+\left|\operatorname{Lip}\left(k_{\infty}^{\epsilon}-k_{\infty}\right)\right|_{L^{2}\left(B_{R}\left(x_{\infty}\right)\right)}<\epsilon .
\end{aligned}
$$


By Proposition $4.4, k_{i}^{\epsilon} g_{i}$ converges weakly to $k_{\infty}^{\epsilon} g_{\infty}$ at a.e. $w \in \Omega_{\epsilon}$. By an argument similar to the proof of Propositions 4.9 and 4.7 , we have

$$
\begin{aligned}
& \left|\int_{B_{R}\left(x_{i}\right)}\left\langle d f_{i}, d k_{i}^{\epsilon}\right\rangle d \underline{\mathrm{vol}}-\int_{B_{R}\left(x_{\infty}\right)}\left\langle d f_{\infty}, d k_{\infty}^{\epsilon}\right\rangle d v\right| \\
& \quad+\left|\int_{B_{R}\left(x_{i}\right)} g_{i} k_{i}^{\epsilon} d \underline{\mathrm{vol}}-\int_{B_{R}\left(x_{\infty}\right)} g_{\infty} k_{\infty}^{\epsilon} d v\right|<\Psi(\epsilon ; n, L, R)
\end{aligned}
$$

for every sufficiently large $i$. Since

$$
\int_{B_{R}\left(x_{i}\right)}\left\langle d f_{i}, d k_{i}^{\epsilon}\right\rangle d \underline{\mathrm{vol}}=\int_{B_{R}\left(x_{i}\right)} g_{i} k_{i}^{\epsilon} d \underline{\mathrm{vol}},
$$

we have

$$
\int_{B_{R}\left(x_{\infty}\right)}\left\langle d f_{\infty}, d k_{\infty}\right\rangle d v=\int_{B_{R}\left(x_{\infty}\right)} g_{\infty} k_{\infty} d v \pm \Psi(\epsilon ; n, L, R) .
$$

By letting $\epsilon \rightarrow 0$, we have the assertion.

We shall recall the notion of (2-) harmonic for Lipschitz functions on Ricci limit spaces. For a Lipschitz function $f$ on $B_{R}\left(x_{\infty}\right)$, we say that $f$ is harmonic on $B_{R}\left(x_{\infty}\right)$ if

$$
\int_{B_{R}\left(x_{\infty}\right)}|d f|^{2} d v \leq \int_{B_{R}\left(x_{\infty}\right)}|d(f+k)|^{2} d v
$$

for every Lipschitz function $k$ on $B_{R}\left(x_{\infty}\right)$, which has compact support. We remark that the notion of harmonic function for $H_{1.2}$-functions is well defined. See Section 7 in [2]. See also [15, 18-20]. The following corollary follows from Corollaries 4.5 and 4.6 directly. See also [11].

Corollary 4.7. Let $R$ be a positive number, $f_{i}$ a harmonic function on $B_{R}\left(x_{i}\right)$ for every $i<\infty$, and $f_{\infty}$ a Lipschitz function on $B_{R}\left(x_{\infty}\right)$ with $\sup _{i} \operatorname{Lip} f_{i}<\infty$. Assume that $f_{i} \rightarrow f_{\infty}$ on $B_{R}\left(x_{\infty}\right)$. Then, we have $\left(f_{i}, d f_{i}\right)$ $\rightarrow\left(f_{\infty}, d f_{\infty}\right)$ on $B_{R}\left(x_{\infty}\right)$. Moreover, we have

$$
\int_{B_{R}\left(x_{\infty}\right)}\left\langle d f_{\infty}, d k_{\infty}\right\rangle d v=0
$$

for every Lipschitz function $k_{\infty}$ on $B_{R}\left(x_{\infty}\right)$, which has compact support. Especially, $f_{\infty}$ is harmonic on $B_{R}\left(x_{\infty}\right)$. 


\section{Acknowledgments}

The author would like to express his deep gratitude to Professor Kenji Fukaya and Professor Tobias Holck Colding for warm encouragement and their numerous suggestions and advice. He is grateful to Professor Takashi Shioya for giving many valuable suggestions. He wishes to thank the referees for valuable suggestions, comments and for pointing out a valuable reference [27]. This work was done during the stay at MIT; he also thanks to them and all members of Informal Geometry Seminar in MIT for warm hospitality and for giving nice environment. He was supported by Grant-in-Aid for Research Activity Start-up 22840027 from JSPS. He was also supported by GCOE "Fostering top leaders in mathematics", Kyoto University.

\section{Appendix A. A proof of Claim 3.15}

In this appendix, we shall give a proof of Claim 3.15. Define functions $\pi_{1}, f_{r}^{A}$ on $\mathbf{R}^{k}$ by $\pi_{1}\left(\left(x_{1}, \ldots, x_{k}\right)\right)=x_{1}, f_{r}^{A}(x)=H^{k-1}\left(\bar{B}_{r}(x) \cap A \cap \pi_{1}^{-1}\left(\pi_{1}(x)\right)\right) 1_{A}(x)$. We remark that by the definition of $s l_{1}-\operatorname{Leb} A$,

$$
\begin{aligned}
s l_{1} & -\operatorname{Leb} A \\
& =\left\{a=\left(a_{1}, \ldots, a_{k}\right) \in A ; \liminf _{r \rightarrow 0} \frac{H^{k-1}\left(\bar{B}_{r}(a) \cap A \cap \pi_{1}^{-1}\left(\pi_{1}(a)\right)\right)}{\omega_{k-1} r^{k-1}}=1\right\} .
\end{aligned}
$$

First, assume that $A$ is compact.

Claim A.1. The function $f_{r}^{A}$ is an upper semi-continuous function on $\mathbf{R}^{k}$. Especially, $f_{r}^{A}$ is a $H^{k}$-measurable function.

Proof. Let $\left\{x_{i}\right\}_{1 \leq i \leq \infty}$ be a sequence of points in $\mathbf{R}^{k}$ with $x_{i} \rightarrow x_{\infty}$. It suffices to check that $\limsup _{i \rightarrow \infty} f_{r}^{A}\left(x_{i}\right) \leq f_{r}^{A}\left(x_{\infty}\right)$ under the assumption: $x_{j} \in A$ for every $j$. Fix $\delta>0$. Let $\{n(i)\}_{i \in \mathbf{N}}$ be a subsequence of $\mathbf{N}$ satisfying $\lim _{j \rightarrow \infty} H^{k-1}\left(\bar{B}_{r}\left(x_{n(j)}\right) \cap A \cap \pi_{1}^{-1}\left(\pi_{1}\left(x_{n(j)}\right)\right)\right)=\lim \sup _{i \rightarrow \infty} H^{k-1}\left(\bar{B}_{r}\left(x_{i}\right) \cap\right.$ $\left.A \cap \pi_{1}^{-1}\left(\pi_{1}\left(x_{i}\right)\right)\right)$. On the other hand, since $\left\{\bar{B}_{r}\left(x_{n(j)}\right) \cap A \cap \pi_{1}^{-1}\left(\pi_{1}\left(x_{n(j)}\right)\right)\right\}_{j}$ is precompact with respect to the Hausdroff distance on $\mathbf{R}^{k}$, without loss of generality, we can assume that there exists a compact subset $K_{\infty}$ of $\mathbf{R}^{k}$ such that $\bar{B}_{r}\left(x_{n(j)}\right) \cap A \cap \pi_{1}^{-1}\left(\pi_{1}\left(x_{n(j)}\right)\right)$ converges to $K_{\infty}$ with respect to the Hausdorff distance on $\mathbf{R}^{k}$. Then, it is easy to check $K_{\infty} \subset \bar{B}_{r}\left(x_{\infty}\right) \cap A \cap$ $\pi_{1}^{-1}\left(\pi_{1}\left(x_{\infty}\right)\right)$. There exists a finite collection $\left\{B_{r_{i}}\left(y_{i}\right)\right\}_{1 \leq i \leq N}$ such that $r_{i} \ll$ $\delta, \bar{B}_{r}\left(x_{\infty}\right) \cap A \cap \pi_{1}^{-1}\left(\pi_{1}\left(x_{\infty}\right)\right) \subset \bigcup_{i=1}^{N} B_{r_{i}}\left(y_{i}\right)$ and $\mid H^{k-1}\left(\bar{B}_{r}\left(x_{\infty}\right) \cap A \cap \pi_{1}^{-1}\right.$ 
$\left.\left(\pi_{1}\left(x_{\infty}\right)\right)\right)-\sum_{i=1}^{N} \omega_{k-1} r_{i}^{k-1} \mid<\delta$. Since $\bar{B}_{r}\left(x_{\infty}\right) \cap A \cap \pi_{1}^{-1}\left(\pi_{1}\left(x_{\infty}\right)\right)$ is compact, there exists $\tau_{0}>0$ such that $B_{\tau_{0}}\left(\bar{B}_{r}\left(x_{\infty}\right) \cap A \cap \pi_{1}^{-1}\left(\pi_{1}\left(x_{\infty}\right)\right)\right) \subset \bigcup_{i=1}^{N}$ $B_{r_{i}}\left(y_{i}\right)$. Since $\bar{B}_{r}\left(x_{n(j)}\right) \cap A \cap \pi_{1}^{-1}\left(\pi_{1}\left(x_{n(j)}\right)\right) \subset B_{\tau_{0}}\left(K_{\infty}\right)$ for every sufficiently large $j$, we have $\bar{B}_{r}\left(x_{n(j)}\right) \cap A \cap \pi_{1}^{-1}\left(\pi_{1}\left(x_{n(j)}\right)\right) \subset \bigcup_{i=1}^{N} B_{r_{i}}\left(y_{i}\right)$. Thus, we have $H^{k-1}\left(\bar{B}_{r}\left(x_{n(j)}\right) \cap A \cap \pi_{1}^{-1}\left(\pi_{1}\left(x_{n(j)}\right)\right)\right) \leq \sum_{i=1}^{N} H^{k-1}\left(\bar{B}_{r}\left(y_{i}\right) \cap \pi_{1}^{-1}\left(\pi_{1}\right.\right.$ $\left.\left.\left(x_{n(j)}\right)\right)\right) \leq \sum_{i=1}^{N} \omega_{k-1} r^{k-1} \leq H^{k-1}\left(\bar{B}_{r}\left(x_{\infty}\right) \cap A \cap \pi_{1}^{-1}\left(\pi_{1}\left(x_{\infty}\right)\right)\right)+\delta$ for every sufficiently large $j$. Therefore, we have Claim A.1.

By Claim A.1, we have statement 1 in Claim 3.15. Statement 2 follows from the Lebesgue differentiation theorem on Euclidean spaces. Finally, by Fubini's theorem, we have

$$
H^{k}\left(A \backslash s l_{1}-\operatorname{Leb} A\right)=\int_{\mathbf{R}} H^{k-1}\left(A \cap\left(\{t\} \times \mathbf{R}^{k-1}\right) \backslash s l_{1}-\operatorname{Leb} A\right) d t=0 .
$$

Thus, we have Statement 3. Therefore, we have Claim 3.15 if $A$ is compact.

We shall give a proof of Claim 3.15 in the general case. Fix $R>0$. There exists a sequence of compact subsets $\left\{K_{i}\right\}_{i}$ of $B_{R}\left(0_{k}\right) \cap A$ such that $H^{k}\left(B_{R}\left(0_{k}\right) \cap A \backslash K_{i}\right) \rightarrow 0$. Then, we have $s l_{1}-\operatorname{Leb} K_{i} \subset s l_{1}-\operatorname{Leb}\left(B_{R}\left(0_{k}\right)\right.$ $\cap A)$. Thus, we have $H^{k}\left(B_{R}\left(0_{k}\right) \cap A \backslash s l_{1}-\operatorname{Leb}\left(B_{R}\left(0_{k}\right) \cap A\right)\right) \leq H^{k}\left(B_{R}\left(0_{k}\right)\right.$ $\left.\cap A \backslash s l_{1}-\operatorname{Leb}_{i}\right) \leq H^{k}\left(B_{R}\left(0_{k}\right) \cap A \backslash K_{i}\right)+H^{k}\left(K_{i} \backslash s l_{1}-\operatorname{Leb} K_{i}\right) \stackrel{i \rightarrow \infty}{\rightarrow} 0$ as an outer measure. Thus, $s l_{1}-\operatorname{Leb}\left(B_{R}(0) \cap A\right)$ is a $H^{k}$-measurable set. Since $s l_{1}-\operatorname{Leb} A=\bigcup_{N \in \mathbf{N}}\left(s l_{1}-\operatorname{Leb}\left(A \cap B_{N}(0)\right)\right)$, we have Statement 1 in Claim 3.15. By the Lebesgue differentiation theorem and Fubini's theorem, we have Statements 2 and 3. Thus, we have Claim 3.15.

\section{References}

[1] J. Cheeger, Differentiability of Lipschitz functions on metric measure spaces, Geom. Funct. Anal. 9 (1999), 428-517.

[2] J. Cheeger, Degeneration of Riemannian metrics under Ricci curvature bounds, Lezioni Fermiane, Scuola Normale Superiore, Pisa (2001).

[3] J. Cheeger and T.H. Colding, Lower bounds on Ricci curvature and the almost rigidity of warped products, Ann. Math. 144 (1996), 189-237.

[4] J. Cheeger and T.H. Colding, On the structure of spaces with Ricci curvature bounded below, I, J. Differ. Geom. 45 (1997), 406-480.

[5] J. Cheeger and T.H. Colding, On the structure of spaces with Ricci curvature bounded below, II, J. Differ. Geom. 54 (2000), 13-35. 
[6] J. Cheeger and T.H. Colding, On the structure of spaces with Ricci curvature bounded below, III, J. Differ. Geom. 54 (2000), 37-74.

[7] S.Y. Cheng and S.T. Yau, Differential equations on Riemannian manifolds and their geometric applications, Commun. Pure Appl. Math. 28 (1975), 333-354.

[8] T.H. Colding, Ricci curvature and volume convergence, Ann. Math. 145 (1997), 477-501.

[9] T.H. Colding and W.P. Minicozzi II, Harmonic functions on manifolds, Ann. Math. 146 (1997), 725-747.

[10] T.H. Colding and W.P. Minicozzi II, Liouville theorems for harmonic sections and applications, Commun. Pure Appl. Math. 51 (1998), $113-138$.

[11] Y. Ding, Heat kernels and Green's functions on limit spaces, Commun. Anal. Geom. 10 (2002), 475-514.

[12] H. Federer, Geometric measure theory, Springer, Berlin-New York, 1969.

[13] K. Fukaya, Collapsing of Riemannian manifolds and eigenvalues of the laplace operator, Invent. Math. 87 (1987), 517-547.

[14] K. Fukaya, Hausdorff convergence of Riemannian manifolds and its applications, Recent topics in differential and analytic geometry, Adv. Stud. Pure Math. 18-I, Academic Press, Boston, MA, 1990, pp. 143238.

[15] M. Fukushima, Dirichlet forms and Markoff processes, North-Holland, Amsterdam 1980.

[16] D. Gilbarg and N. Trudinger, Elliptic partial differential equations of second order, Reprint of the 1998 edition, Classics in Mathematics, Springer-Verlag, Berlin 2001.

[17] M. Gromov, Metric structures for Riemannian and non-Riemannian spaces, Birkhauser Boston Inc, Boston, MA, 1999, Based on the 1981 French original, With appendices by M. Katz, P. Pansu, and S. Semmes, Translated from the French by Sean Michael Bates, MR 85e:53051.

[18] P. Hajlasz, Sobolev spaces on an arbitrary metric space, J. Potential Anal. 5 (1995), 403-415. 
[19] P. Hajlasz and P. Koskela, Sobolev meets Poincare, C. R. Acad. Sci. Paris 320 (1995), 1211-1215.

[20] J. Heinonen, T. Kilpeläinen and O. Martio, Nonlinear potential theory for degenerate elliptic equations, Clarendon Press, Oxford, Tokyo, New York, 1993.

[21] S. Honda, Ricci curvature and almost spherical multi-suspension, Tohoku Math. J. 61 (2009), 499-522.

[22] S. Honda, Bishop-Gromov type inequality on Ricci limit spaces, J. Math. Soc. Japan, 63 (2011), 419-442.

[23] S. Honda, On Low Dimensional Ricci limit spaces, submitted.

[24] S. Honda, Harmonic functions on asymptotic cones with Euclidean volume growth, preprint.

[25] S. Honda in preparation.

[26] A. Kasue, Convergence of Riemannian manifolds and Laplace operators, I, Ann. Inst. Fourier 52 (2002), 1219-1257.

[27] A. Kasue, Convergence of Riemannian manifolds and Laplace operators, II, Potential Anal. 24 (2006), 137-194.

[28] A. Kasue and H. Kumura, Spectral convergence of Riemannian manifolds, Tohoku Math. J. 4 (1994), 147-179.

[29] A. Kasue and H. Kumura, Spectral convergence of Riemannian manifolds II, Tohoku Math. J. 2 (1996), 71-120.

[30] K. Kuwae and T. Shioya, Convergence of spectral structures: a functional analytic theory and its applications to spectral geometry, Commun. Anal. Geom. 11 (2003), 599-673.

[31] P. Li, The theory of harmonic functions and its relation to geometry, Proceedings of Symposium on Pure Mathematics American Mathematical Society vol. 54, Part 1, ed. R. Green and S. T. Yau, 1993.

[32] P. Li, Harmonic functions on complete Riemannian manifolds, in Handbook of Gemetric Analysis, I, Advanced Lectures in Mathematics, International Press, 7 (2008), 195-227.

[33] P. Li and L-F. Tam, Green's functions, harmonic functions, and volume comparison, J. Differ. Geom. 41 (1995), 277-318. 
[34] J. Lott, Optimal transport and Ricci curvature for metric-measure spaces, in Surveys in Differential Geometry, XI, Metric and Comparison Geometry, eds. J. Cheeger and K. Grove, International Press, Somerville, MA, 2007, pp. 229-257.

[35] C.B. Morrey Jr., Multiple integrals in the calculus of variations, Springer, New York, 1966.

[36] R. Schoen and S.T. Yau, Lectures on differential geometry, International Press, 1995.

[37] N. Shanmugalingam, Harmonic functions on metric spaces, Illinois J. Math. 45 (2001), 1021-1050.

[38] L.M. Simon, Lectures on geometric measure theory, Proceedings of the Center for Mathematical Analysis 3, Australian National University, 1983.

[39] S.T. Yau, Harmonic functions on complete Riemannian manifolds, Commun. Pure and Appl. Math. 28 (1975), 201-228.

[40] S.T. Yau, Some function-theoretic properties of complete Riemannian manifold and their applications to geometry, Indiana Univ. Math. J. 25 (1976), 659-670.

Research Institute for Mathematical Sciences

KYOTO UNIVERSITY

КҮОТО 606-8502

JAPAN

E-mail address: honda@kurims.kyoto-u.ac.jp

ReCeIVed May 11, 2010 